\title{
Global Gauge Anomalies in Two-Dimensional Bosonic Sigma Models
}

\author{
Krzysztof Gawȩdzki $^{1}$, Rafał R. Suszek ${ }^{2}$, Konrad Waldorf ${ }^{3}$ \\ ${ }^{1}$ Laboratoire de Physique, C.N.R.S., ENS-Lyon, Université de Lyon, 46 Allée d'Italie, 69364 Lyon, France. \\ E-mail:kgawedzk@ens-lyon.fr \\ 2 Department Mathematik, Bereich Algebra und Zahlentheorie, Universität Hamburg, Bundesstraße 55, \\ 20146 Hamburg, Germany \\ 3 Department of Mathematics, University of California, Berkeley, 970 Evans Hall \#3840, Berkeley, \\ CA 94720, USA
}

Received: 23 March 2010 / Accepted: 3 June 2010

Published online: 21 November 2010 - () The Author(s) 2010. This article is published with open access at Springerlink.com

\begin{abstract}
We revisit the gauging of rigid symmetries in two-dimensional bosonic sigma models with a Wess-Zumino term in the action. Such a term is related to a background closed 3-form $H$ on the target space. More exactly, the sigma-model Feynman amplitudes of classical fields are associated to a bundle gerbe with connection of curvature $H$ over the target space. Under conditions that were unraveled more than twenty years ago, the classical amplitudes may be coupled to the topologically trivial gauge fields of the symmetry group in a way which assures infinitesimal gauge invariance. We show that the resulting gauged Wess-Zumino amplitudes may, nevertheless, exhibit global gauge anomalies that we fully classify. The general results are illustrated on the example of the WZW and the coset models of conformal field theory. The latter are shown to be inconsistent in the presence of global anomalies. We introduce a notion of equivariant gerbes that allow an anomaly-free coupling of the Wess-Zumino amplitudes to all gauge fields, including the ones in non-trivial principal bundles. Obstructions to the existence of equivariant gerbes and their classification are discussed. The choice of different equivariant structures on the same bundle gerbe gives rise to a new type of discrete-torsion ambiguities in the gauged amplitudes. An explicit construction of gerbes equivariant with respect to the adjoint symmetries over compact simply connected simple Lie groups is given.
\end{abstract}

\section{Contents}

1. Introduction . . . . . . . . . . . . . . . . . . . 514

2. Wess-Zumino Feynman Amplitudes . . . . . . . . . . . . . . . . 516

$2.12 \mathrm{D}$ Wess-Zumino action and gerbes $\ldots \ldots \ldots \ldots 16 \ldots$

2.2 Rigid symmetries of Wess-Zumino amplitudes . . . . . . . . . . . 517

3. Coupling to Topologically Trivial Gauge Fields . . . . . . . . . . . . . . 519

3.1 Gauging prescription . . . . . . . . . . . . . . . 519

3.2 Equivariant-cohomology interpretation . . . . . . . . . . 521 
3.3 More equivariance properties . . . . . . . . . . . . . . . . . 522

4. Global Gauge Anomalies . . . . . . . . . . . . . . . . . . . . . 524

4.1 General gauge transformations . . . . . . . . . . . . . . . 524

4.2 Global gauge anomalies in WZW amplitudes . . . . . . . . . . 527

4.3 Anomalies and WZW partition functions . . . . . . . . . . . . 530

4.4 Implications for coset models . . . . . . . . . . . . . . . . . . . 534

5. Coupling to General Gauge Fields . . . . . . . . . . . . . . . . . 537

5.1 Equivariant gerbes . . . . . . . . . . . . . . . . . . . . 537

$5.2 \mathrm{WZ}$ amplitudes with topologically non-trivial gauge fields . . . . . . . 539

5.3 General gauge invariance . . . . . . . . . . . . . . . . 542

6. Obstructions and Classification of Equivariant Structures . . . . . . . . . . 544

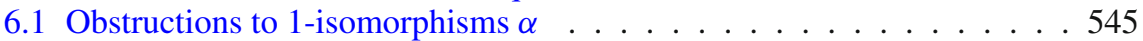

6.2 Local description of gerbes . . . . . . . . . . . . . . . . . 546

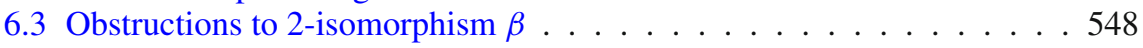

6.4 Obstructions to the commutativity of diagram (5.1) $\ldots \ldots \ldots \ldots 50$

6.5 Classification of equivariant structures . . . . . . . . . . . 551

6.6 Ambiguity of gauged amplitudes _ . . . . . . . . . . . . . 554

6.7 Fixed-point resolved coset partition functions . . . . . . . . . . . 557

7. Ad-Equivariant WZW Gerbes Over Simply Connected Groups . . . . . 558

7.1 WZW gerbes over compact simply connected simple Lie groups . . . . 559

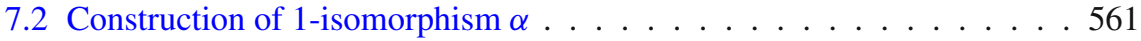

7.3 Construction of 2 -isomorphism $\beta \ldots \ldots \ldots \ldots 65$

7.4 Commutativity of diagram (5.1) $\ldots \ldots \ldots 67$

8. Conclusions . . . . . . . . . . . . . . . . . . . . . 568

Appendices . . . . . . . . . . . . . . . . . . . . . . . . . . . . . . . 569

1 Proof of Proposition $3.1 \ldots \ldots$. . . . . . . . . . . . . . . . 569

2 Proof of Lemma $3.13 \ldots \ldots$. . . . . . . . . . . . . . . . 571

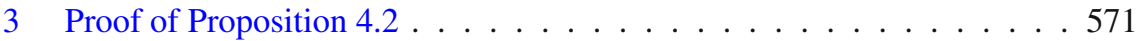

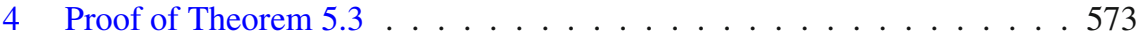

5 Proof of Lemma $5.4 \ldots \ldots \ldots$. . . . . . . . . . . . . . . . . 575

6 Construction of flat gerbes from characters . . . . . . . . . . 576

7 Behavior of isomorphism $\alpha$ under groupoid multiplication . . . . . 576

8 Commutativity of diagram (7.74) . . . . . . . . . . . . 577

9 Proof of the equality of isomorphisms (7.76) and (7.77) $\ldots \ldots \ldots 578$

References . . . . . . . . . . . . . . . . . . . . . . . . 579

\section{Introduction}

Gauge invariance constitutes one of the basic principles underlying the theoretical description of physical reality. The occurrence of its violations, called gauge anomalies [3], in certain models of quantum field theory with chiral fermions yields a powerful selection principle for the model building in high energy physics [56]. Gauge anomalies may describe violations of infinitesimal gauge invariance, or, if the latter holds, the breakdown of invariance under large gauge transformations not homotopic to identity [58]. The second type goes under the name of global gauge anomalies. Anomalies similar to the ones in theories with chiral fermions occur also in effective bosonic models describing the low energy sector [51]. Such effective theories contain Wess-Zumino (WZ) terms in the action [57], see, e.g., the review [46]. The emergence of global gauge anomalies in bosonic theories with WZ terms on the Euclidean space-time compactified 
to the four-dimensional sphere was extensively analyzed following the work [58], see [11].

Starting with Witten's paper [59] on non-Abelian bosonization, the two-dimensional Wess-Zumino actions for bosonic sigma models with Lie-group targets were studied quite thoroughly in the context of the Wess-Zumino-Witten (WZW) models of conformal field theory (CFT). In the latter setting, the problem how to gauge rigid symmetries was solved, at least in the simplest cases, almost from the very start [8]. Nevertheless, the general question about the coupling of two-dimensional Wess-Zumino actions to gauge fields in a way invariant under infinitesimal gauge transformations was posed and answered only a few years later in [37] and in [36]. Besides, this was done only for topologically trivial gauge fields described by global 1 -forms on the worldsheet. The conditions that permit such gauging and the obstructions to their fulfillment were subsequently interpreted in $[13,14]$ in terms of equivariant cohomology, as first indicated in [60], see also [61]. The issue of general gauge invariance of gauged two-dimensional WZ actions was addressed only very briefly at the end of [13] and, in the context of the $T$-duality, in $[34,35]$. We make it the main topic of the present study.

A convenient tool to treat topological intricacies of Wess-Zumino actions $[1,17]$ on closed two-dimensional worldsheets is provided by the theory of bundle gerbes with connection $[43,44]$. For topologically trivial gauge fields, we identify the global gauge anomalies of gauged WZ actions as the isomorphism classes of certain flat gerbes over the product of the symmetry group $\Gamma$ and the target space $M$. Such isomorphism classes correspond to the classes in the cohomology group $H^{2}(\Gamma \times M, U(1))$ that may often be calculated explicitly. In particular, we show how to do it in the case of WZW models. This permits us to prove that, after the gauging of an adjoint symmetry, some of bulk WZW models with non-simply connected target groups exhibit global gauge anomalies. The latter lead to the inconsistency of the corresponding coset models of CFT [29,30] realized as gauged WZW models with the gauge fields integrated out $[2,21,22,40]$. This is the main surprise resulting from our study.

We also address the problem of the coupling of WZ actions to topologically non-trivial gauge fields given by connections in non-trivial principal bundles of the symmetry group. It was indicated in [33] that such a coupling plays an important role in the construction of consistent coset theories. It seems also important in the $T$-duality [34]. We show that the existence of certain equivariant structures on gerbes, considered already before for discrete symmetry groups in [27], enables a non-anomalous coupling to all gauge fields and we analyze in a cohomological language the obstructions to the existence of such structures and their classification. An explicit construction of all non-equivalent equivariant structures relative to the adjoint symmetries on gerbes relevant for the WZW models with compact simply connected target groups is given. Different choices of the equivariant structure lead to the amplitudes with topologically non-trivial gauge fields that differ by phases that are given by characters of (a subgroup of) the fundamental group of the (connected) symmetry group. The appearance of such discrete-torsionlike phases in the coset model sectors with topologically non-trivial gauge fields was envisaged in [33]. We discuss its implication on the resolution of the field-identification problem [16] in general coset models.

The paper is organized as follows. In Sect. 2, we recall the role of bundle gerbes in the definition of the Feynman amplitudes of two-dimensional sigma models with a WZ action (in Sect. 2.1) and we characterize rigid symmetries of such amplitudes (in Sect. 2.2). Section 3 is devoted to the coupling of WZ actions to topologically trivial gauge fields. In Sect. 3.1, we recall the old result of Jack-Jones-Mohammedi-Osborn [37] and Hull-Spence [36] describing the coupling of a WZ action to the gauge fields 
of its symmetry group. In Sect. 3.2, we review the interpretation, due to Witten [60] and Figueroa-O'Farrill-Stanciu [13,14], of the conditions that permit such gauging in terms of the Cartan model of equivariant cohomology, and, in Sect. 3.3, we study further implications of those conditions. Section 4 is devoted to global gauge anomalies in theories with a WZ action coupled to topologically trivial gauge fields. Section 4.1 derives the transformation law of the Feynman amplitudes under general gauge transformations and identifies, in cohomological terms, the obstruction to the invariance of the amplitudes under large gauge transformations not homotopic to identity. The general discussion is illustrated in Sect. 4.2 by the example of WZW models with non-simply connected target groups and gauged adjoint symmetry. In this case, the presence or the absence of global gauge anomalies is decided by a simple condition stated in Proposition 4.8. In Sect. 4.3, we show that our results are consistent with the known solution for the partition functions of WZW models and in Sect. 4.4, we examine the toroidal partition functions of the coset models in the presence of global anomalies, pointing to the inconsistency of such models. Section 5 is devoted to the coupling of WZ actions to topologically non-trivial gauge fields. In Sect. 5.1, we define gerbes with equivariant structure. In Sect. 5.2, we describe how to use such structures to define WZ amplitudes coupled to gauge fields with arbitrary topology. The general gauge invariance of such amplitudes is proven in Sect. 5.3. In Sect. 6, we study subsequently the obstructions to the existence of the three layers of an equivariant structure on gerbes (in Sects. 6.1, 6.3 and 6.4). We use the local-data description of gerbes that is recalled in Sect. 6.2. The classification of equivariant gerbes is discussed in Sect. 6.5. Sect. 6.6 examines the change of the WZ amplitudes induced by a change of the equivariant structure of the gerbe and Sect. 6.7 studies the reflection of such changes in the coset toroidal partition functions. Next Sect. 7 contains an explicit construction of equivariant structures relative to the adjoint symmetry on gerbes and relevant for the WZW models with compact simple and simply connected target groups. In Sect. 7.1, we recall the construction of the corresponding gerbes over the target groups and in Sects. 7.2, 7.3 and 7.4, we build the different layers of the equivariant structure. Finally, Sect. 8 summarizes our results and discusses directions for future work. More technical proofs are collected in nine Appendices.

When the present work was finished we learnt that a similar concept of equivariant gerbes was recently discussed in [45] and a different one, earlier, in [31].

\section{Wess-Zumino Feynman Amplitudes}

2.1. $2 D$ Wess-Zumino action and gerbes. Let $M$ be a smooth manifold and $H$ a closed 3-form on $M$. 2-forms $B$ such that $d B=H$ provide the background Kalb-Ramond fields for the two-dimensional sigma model with target space $M$. We shall be mostly interested in situations when $H$ is not an exact form so that the 2-forms $B$ exist only locally. The classical fields of the sigma model are smooth maps $\varphi: \Sigma \rightarrow M$, where $\Sigma$, called the worldsheet, is a compact surface, not necessarily connected, that will be assumed closed and oriented here. The Kalb-Ramond field contributes to the sigma-model action functional and to the Feynman amplitude of the field configuration $\varphi$ the Wess-Zumino terms which, for the global 2-form $B$, are equal to

$$
S_{W Z}(\varphi):=\int_{\Sigma} \varphi^{*} B \quad \text { and } \quad A_{W Z}(\varphi):=\exp \left(i S_{W Z}(\varphi)\right)=\exp \left(i \int_{\Sigma} \varphi^{*} B\right)
$$


respectively, in the units where the Planck constant $\hbar=1$. The contribution to the Feynman amplitudes may be defined more generally if, instead of a global 2-form $B$, one is given a bundle gerbe with unitary connection $\mathcal{G}$ over $M$, called simply gerbe below, with curvature equal to the closed 3-form $H$ [43]. Such gerbes are precisely the geometric objects that allow to define a $U(1)$-valued holonomy $\operatorname{Hol}_{\mathcal{G}}(\varphi)$ of maps $\varphi: \Sigma \rightarrow M$, and one sets

$$
\boldsymbol{A}_{W Z}(\varphi):=\operatorname{Hol}_{\mathcal{G}}(\varphi) .
$$

In particular, if $H=d B$ for a global 2-form on $M$, there exists a gerbe $\mathcal{I}_{B}$ with curvature $H$, canonically associated to $B$, such that

$$
\operatorname{Hol}_{\mathcal{I}_{B}}(\varphi)=\exp \left(i \int_{\Sigma} \varphi^{*} B\right) .
$$

Gerbes with curvature $H$ exist if and only if the periods of the closed 3-form $H$ are in $2 \pi \mathbb{Z}$. In particular, $H$ is not required to be an exact form.

The basic property of the holonomy of a gerbe $\mathcal{G}$ with curvature $H$ is that it is a (Cheeger-Simons) differential character. This means that if $\tilde{\Sigma}$ is a compact oriented 3-manifold with boundary $\partial \tilde{\Sigma}=\Sigma$, and if $\tilde{\varphi}: \tilde{\Sigma} \rightarrow M$, then, for $\varphi=\left.\tilde{\varphi}\right|_{\Sigma}$,

$$
\operatorname{Hol}_{\mathcal{G}}(\varphi)=\exp \left(i \int_{\tilde{\Sigma}} \tilde{\varphi}^{*} H\right) .
$$

Consequently, the gerbe holonomy is fully determined for the boundary values of fields $\tilde{\varphi}$ by the gerbe curvature $H$. On the other hand, taking a 3-dimensional ball for $\tilde{\Sigma}$ ones infers easily that the gerbe holonomy determines the gerbe curvature $H$. The converse is true only if the homology group $\mathrm{H}_{2}(M)$ is trivial.

The (bundle) gerbes (with unitary connection) $\mathcal{G}$ over $M$ form a 2-category $\operatorname{Grb}^{\nabla}(M)$ with 1-morphisms between gerbes and 2-morphisms between 1-morphisms [50]. Below, we shall denote by $I d$ as well the identity maps between spaces as the identity 1-isomorphisms between gerbes and the identity 2-isomorphisms between 1-isomorphisms, with the meaning of the symbol that should be clear from the context. Gerbes $\mathcal{G}$ possess duals $\mathcal{G}^{*}$ with opposite curvature and inverse holonomy, tensor products $\mathcal{G}_{1} \otimes \mathcal{G}_{2}$ with added curvatures and multiplied holonomies, and pullbacks $f^{*} \mathcal{G}$ under smooth maps $f$ of the underlying base manifolds with curvatures related by the pullback of 3 -forms and the same holonomies of maps $\varphi$ related by the composition with $f$. Up to 1-isomorphisms, gerbes are classified by their holonomy. Indeed, two gerbes with the same curvature differ, up to a 1-isomorphism, by a tensor factor that is a flat gerbe (i.e. has vanishing curvature). Their holonomies differ by the flat gerbe holonomy factor that determines a cohomology class in $H^{2}(M, U(1))=H_{o m}\left(H_{2}(M), U(1)\right)$. All the elements of $H^{2}(M, U(1))$ may be obtained this way.

2.2. Rigid symmetries of Wess-Zumino amplitudes. Rigid symmetries of sigma models are induced by transformations of the target space. Let $\Gamma$ be a Lie group that, in general, will not be assumed to be connected or simply connected. Suppose now that $M$ is a $\Gamma$-space, i.e. that we are given a smooth action $\ell: \Gamma \times M \rightarrow M$ of $\Gamma$ on $M$. We shall variably write $\ell(\gamma, m):=\ell_{\gamma}(m):=r_{m}(\gamma):=\gamma m$. The infinitesimal action of the Lie algebra $\mathbf{g}$ of $\Gamma$ on $M$ is induced by the vector fields $\bar{X}$ for $X \in \mathbf{g}$, where $\bar{X}(m)=\left.\frac{d}{d t}\right|_{t=0} \mathrm{e}^{-t X} m$. 
The assignment preserves the commutators: $[\bar{X}, \bar{Y}]=\overline{[X, Y]}$. We would like to determine when the WZ Feynman amplitudes are invariant under this action. Below, $\iota \mathcal{X}$ will denote the contraction with the vector field $\mathcal{X}$, and $\mathcal{L}_{\mathcal{X}}=d \iota \mathcal{X}+\iota \mathcal{X} d$ the Lie derivative with respect to it.

Lemma 2.1. The variation of the gerbe holonomy of maps $\varphi: \Sigma \rightarrow M$ under the infinitesimal action of $X \in \mathbf{g}$ is given by the formula

$$
\left.\frac{d}{d t}\right|_{t=0} \operatorname{Hol}_{\mathcal{G}}\left(\mathrm{e}^{-t X} \varphi\right)=\left(i \int_{\Sigma} \varphi^{*} \iota_{\bar{X}} H\right) \operatorname{Hol}_{\mathcal{G}}(\varphi) .
$$

Proof. The relation (2.4) implies that

$$
\begin{aligned}
& \operatorname{Hol}_{\mathcal{G}}\left(\mathrm{e}^{-t X} \varphi\right) \\
& \quad=\exp \left(i \int_{[0,1] \times \Sigma} \tilde{\varphi}_{t}^{*} H\right) \operatorname{Hol}_{\mathcal{G}}(\varphi)=\exp \left(i \int_{[0,1] \times \Sigma} \tilde{\psi}_{t}^{*} p r_{2}^{*} H\right) \operatorname{Hol}_{\mathcal{G}}(\varphi)
\end{aligned}
$$

for $\tilde{\varphi}_{t}(s, x)=\mathrm{e}^{-s t X} \varphi(x), \tilde{\psi}_{t}(s, x)=\left(s, \tilde{\varphi}_{t}(s, x)\right)$ and $\operatorname{pr}_{2}(s, m)=m$. Differentiation of the right hand side with respect to $t$ gives

$$
\begin{aligned}
& \left.\frac{d}{d t}\right|_{t=0} \operatorname{Hol}_{\mathcal{G}}\left(\mathrm{e}^{-t X} \varphi\right) \\
& =\left(i \int_{[0,1] \times \Sigma} \tilde{\psi}_{0}^{*} \mathcal{L}_{\tilde{X}} p r_{2}^{*} H\right) \operatorname{Hol}_{\mathcal{G}}(\varphi)=\left(i \int_{[0,1] \times \Sigma} d \tilde{\psi}_{0}^{*} \iota_{\tilde{X}} p r_{2}^{*} H\right) \operatorname{Hol}_{\mathcal{G}}(\varphi),
\end{aligned}
$$

where $\tilde{X}$ is the vector field on $[0,1] \times M$ such that $\tilde{X}(s, m)=\left.\frac{d}{d t}\right|_{t=0}\left(s, \mathrm{e}^{-s t X} m\right)=$ $s \bar{X}(m)$. The Stokes formula applied to the last integral results in the claim.

Lemma 2.1 implies that the left hand side of Eq. (2.5) vanishes if and only if

$$
\int_{\Sigma} \varphi^{*} \iota_{\bar{X}} H=0
$$

This holds for all $\varphi$ if and only if $\iota_{\bar{X}} H$ is an exact form. We obtain this way

Corollary 2.2. The Feynman amplitudes $\boldsymbol{A}_{W Z}(\varphi)$ are invariant under the infinitesimal action of the Lie algebra $\mathbf{g}$ (or, equivalently, of the connected component of unity $\Gamma_{0} \subset \Gamma$ ) if and only if the 2-forms $\iota_{\bar{X}} H$ are exact for all $X \in \mathbf{g}$.

Note that the exactness of $\iota_{\bar{X}} H$ implies, in particular, that $\mathcal{L}_{\bar{X}} H=0$, i.e. that the curvature 3-form $H$ is invariant under the infinitesimal action of $\mathbf{g}$. Observe also that if $H=d B$ for a global g-invariant 2-form $B$, then $\iota_{\bar{X}} H=-d\left(\iota_{\bar{X}} B\right)$ so that the 2-forms $\iota_{\bar{X}} H$ are exact.

If the group $\Gamma$ is not connected, i.e. $\Gamma \neq \Gamma_{0}$, then the condition for the $\Gamma$-invariance of the WZ Feynman amplitudes is more stringent. Since

$$
\operatorname{Hol}_{\mathcal{G}}(\gamma \varphi)=\operatorname{Hol}_{\ell_{\gamma}^{*} \mathcal{G}}(\varphi)
$$

for $\gamma \in \Gamma$, it follows that $\boldsymbol{A}_{W Z}(\gamma \varphi)=\boldsymbol{A}_{W Z}(\varphi)$ for all $\varphi$ if and only if the gerbes $\ell_{\gamma}^{*} \mathcal{G}$ and $\mathcal{G}$ have the same holonomy. In particular, they have to have the same curvature: $\ell_{\gamma}^{*} H=H$. Since the holonomy determines the 1-isomorphism class of a gerbe, we obtain 
Corollary 2.3. The Feynman amplitudes $\boldsymbol{A}_{W Z}(\varphi)$ are invariant under the action of $\Gamma$ if and only if the gerbes $\ell_{\gamma}^{*} \mathcal{G}$ and $\mathcal{G}$ are 1-isomorphic for all $\gamma \in \Gamma$.

Remark 2.4. In most applications, the sigma-model target manifold $M$ is equipped with a Riemannian metric $g_{M}$ and the Feynman amplitudes contain also a factor with the standard sigma-model action $S(\varphi)=\|d \varphi\|_{L^{2}}^{2}$ defined with the help of $g_{M}$ and of a Riemannian metric $g_{\Sigma}$ on the worldsheet. In that situation a group $\Gamma$ of rigid symmetries that leaves the total amplitudes unchanged for arbitrary Riemann surfaces as worldsheets has to preserve additionally the target metric $g_{M}$ so that, in particular, $\bar{X}$ (for $X \in \mathbf{g}$ ) are Killing vector fields.

\section{Coupling to Topologically Trivial Gauge Fields}

A natural question arises whether g-invariant Feynman amplitudes $\boldsymbol{A}_{W Z}(\varphi)$ may be gauged, i.e. coupled to gauge fields in a gauge-invariant way. First, we shall discuss the case of topologically trivial gauge fields given by global g-valued 1-forms $A$ on the worldsheet $\Sigma$. Such forms may be viewed as connections on the trivial principal $\Gamma$-bundle $\Sigma \times \Gamma$ for group $\Gamma$ with Lie algebra $\mathbf{g}$.

3.1. Gauging prescription. In the particular instance when the WZ Feynman amplitudes are determined by a global g-invariant 2-form $B$ with $d B=H$, one may realize the gauging by replacing $B$ with its minimally coupled version $B_{A}$ which is a 2 -form on $\Sigma \times M$ :

$$
B_{A}:=\exp \left(-\iota_{\bar{A}}\right) B=B-\iota_{\bar{A}} B+\frac{1}{2} \iota_{\bar{A}}^{2} B
$$

Above, for $X \in \mathbf{g}$ and $\alpha$ a differential form, we define $\iota_{\bar{X} \otimes \alpha}=\alpha \iota_{\bar{X}}$ (omitting the wedge sign for the exterior product of differential forms). The gauged Wess-Zumino action has then the form

$$
S_{W Z}(\varphi, A):=\int_{\Sigma} \phi^{*} B_{A}=S_{W Z}(\varphi)+\int_{\Sigma} \phi^{*}\left(-\iota_{\bar{A}} B+\frac{1}{2} \iota_{\bar{A}}^{2} B\right),
$$

where $\phi=(I d, \varphi): \Sigma \rightarrow \Sigma \times M$. It is well known that the minimal coupling gives an action that is invariant under infinitesimal gauge transformations induced by the maps $\Lambda: \Sigma \rightarrow$ g. This means that

$$
\frac{d}{d t} S_{W Z}\left(\mathrm{e}^{-t \Lambda} \varphi, \mathrm{e}^{-t \Lambda} A\right)=0
$$

where, for $x \in \Sigma$,

$$
\left(\mathrm{e}^{-t \Lambda} \varphi\right)(x)=\mathrm{e}^{-t \Lambda(x)} \varphi(x), \quad\left(\mathrm{e}^{-t \Lambda} A\right)(x)=A d_{\mathrm{e}^{-t \Lambda(x)}} A(x)+\mathrm{e}^{-t \Lambda(x)} d \mathrm{e}^{t \Lambda(x)} .
$$

The invariance (3.3) will also follow from the considerations below.

In the more general case when the Feynman amplitudes $\boldsymbol{A}_{W Z}(\varphi)$ are given by the gerbe holonomy, see Eq. (2.2), one may still postulate that the coupling to the gauge 
fields is realized by terms linear and quadratic in $A$, resulting in the replacement of $\boldsymbol{A}_{W Z}(\varphi)$ by

$$
\boldsymbol{A}_{W Z}(\varphi, A):=\exp \left(i \int_{\Sigma} \phi^{*}\left(-v(A)+\frac{1}{2} u\left(A^{2}\right)\right)\right) \boldsymbol{A}_{W Z}(\varphi),
$$

where $v(X)$ are 1-forms on $M$ depending linearly on $X \in \mathbf{g}, u(X \wedge Y)$ are functions on $M$ depending linearly on $X \wedge Y \in \mathbf{g} \wedge \mathbf{g}$ and, for a form $\alpha$ on $\Sigma, v(X \otimes \alpha):=v(X) \alpha$ and $u((X \wedge Y) \otimes \alpha):=u(X \wedge Y) \alpha$ denote the induced forms on $\Sigma \times M$. Necessary conditions for the consistency of such a coupling were found in [37] and [36]. They are summarized in

Proposition 3.1. The amplitudes $\boldsymbol{A}_{W Z}(\varphi, A)$ defined in (3.5) are invariant under infinitesimal gauge transformations if and only if the 1-forms $v(X)$ satisfy the relations

$$
\iota_{\bar{X}} H=d v(X), \quad \mathcal{L}_{\bar{X}} v(Y)=v([X, Y]), \quad \iota_{\bar{X}} v(Y)=-\iota_{\bar{Y}} v(X)
$$

for all $X, Y \in \mathbf{g}$, with the functions $u$ given by

$$
u(X \wedge Y)=\iota_{\bar{X}} v(Y) .
$$

For completeness, we give in Appendix 1 a proof of this result by arguments close to the original ones of [37] and [36].

Remark 3.2. 1. The 1-forms $v(X)$ satisfying Eqs. (3.6) may be modified by 1 -forms $w(X)$ (also linear in $X$ ) satisfying the homogeneous version of these equations.

2. To make contact with refs. [37] and [36] more explicitly, let us introduce a basis $\left(t^{a}\right)$ of the Lie algebra $\mathbf{g}$ with $\left[t^{a}, t^{b}\right]=f^{a b c} t^{c}$ (the summation convention!), $v\left(t^{a}\right)=: v^{a}$, and $u\left(t^{a} \wedge t^{b}\right)=: u^{a b}$. Denoting by $\iota^{a}$ and $\mathcal{L}^{a}$ the contraction with and the Lie derivative w.r.t. the vector field $\bar{t}^{a}$, the relations (3.6) and (3.7) may be rewritten as

$$
\iota^{a} H=d v^{a}, \quad \mathcal{L}^{a} v^{b}=f^{a b c} v^{c}, \quad \iota^{a} v^{b}=-\iota^{b} v^{a}=u^{a b} .
$$

In view of Proposition 3.1, it will be convenient to introduce a 2-form $\rho_{A}$ on the product manifold $\Sigma \times M$ and a gerbe $\mathcal{G}_{A}$ over the same space by the formulae

$$
\rho_{A}=-v(A)+\frac{1}{2} \iota_{\bar{A}} v(A) \text { and } \mathcal{G}_{A}=\mathcal{I}_{\rho_{A}} \otimes \mathcal{G}_{2} .
$$

Equation (3.5), together with the conditions (3.6) and (3.7) on its entries, may then be summarized in the following

Definition 3.3. Let $\mathcal{G}$ be a gerbe with curvature $H$ over a $\Gamma$-space $M$, and let $v(X)$ be 1-forms on $M$, depending linearly on $X \in \mathbf{g}$, satisfying conditions (3.6). The WessZumino contribution of a field $\varphi: \Sigma \rightarrow M$ to the Feynman amplitude coupled to a gauge field 1-form A on $\Sigma$ is defined as

$$
\boldsymbol{A}_{W Z}(\varphi, A)=\exp \left(i \int_{\Sigma} \phi^{*} \rho_{A}\right) \boldsymbol{A}_{W Z}(\varphi)=\operatorname{Hol}_{\mathcal{G}_{A}}(\phi),
$$

where, as before, $\phi=(I d, \varphi)$.

Remark 3.4. If the gerbe $\mathcal{G}$ is equal to $\mathcal{I}_{B}$ for a g-invariant 2-form $B$ such that $d B=H$, then one may take $v(X)=-\iota_{\bar{X}} B$. In this case, Eq. (3.10) agrees with the minimal coupling (3.2) of the Wess-Zumino action. 
Proposition 3.1 implies immediately

Corollary 3.5. Equation (3.10) defines amplitudes that are invariant under infinitesimal gauge transformations.

Below, we shall need two easy implications of relations (3.6) whose straightforward proof is left to the reader. They will be employed repeatedly below.

Lemma 3.6. Relations (3.6) imply that

$$
\begin{aligned}
\iota_{\bar{X}} \iota_{\bar{Y}} H & =v([X, Y])-d_{\bar{X}} v(Y), \\
\iota_{\bar{X}} \iota_{\bar{Y}} \iota_{\bar{Z}} H & =\iota_{\bar{X}} v([Y, Z])+\iota_{\bar{Z}} v([X, Y])+\iota_{\bar{Y}} v([Z, X]) .
\end{aligned}
$$

3.2. Equivariant-cohomology interpretation. In refs. [13,14], see also [60] and [61], relations (3.6) were interpreted in terms of equivariant cohomology. Let $\Omega(M)$ denote the space of differential forms on $M$. Recall that the Cartan complex for equivariant cohomology is formed of polynomial maps

$$
\text { g } \ni X \longmapsto \hat{\omega}(X) \in \Omega(M)
$$

which satisfy

$$
\mathcal{L}_{\bar{X}} \hat{\omega}(Y)=\left.\frac{d}{d t}\right|_{t=0} \hat{\omega}\left(A d_{\mathrm{e}^{t X}} Y\right) \text { for } X, Y \in \mathbf{g} .
$$

We shall call such maps g-equivariant forms. Note that relation (3.14) holds if and only if

$$
\ell_{\gamma}^{*} \hat{\omega}(Y)=\hat{\omega}\left(A d_{\gamma^{-1}} Y\right)
$$

for $\gamma$ in the connected component $\Gamma_{0}$ of 1 in $\Gamma$. We shall say that a form $\hat{\omega}$ is $\Gamma$-equivariant if the relation (3.15) is satisfied for all $\gamma \in \Gamma$. Of course, the two notions of equivariance coincide if the group $\Gamma$ is connected. The g-equivariant ( $\Gamma$-equivariant) forms make up the complex $\Omega_{\mathbf{g}}^{\bullet}(M)\left(\Omega_{\Gamma}^{\bullet}(M)\right)$ with the $\mathbb{Z}$-grading that adds twice the degree of the polynomial to the degree of the form and with the differential of degree 1 given by the formula

$$
(\hat{d} \hat{\omega})(X)=d \hat{\omega}(X)-\iota_{\bar{X}} \hat{\omega}(X)
$$

The following result was obtained in $[13,14]$ :

Proposition 3.7. A g-equivariantly closed 3-form $\hat{H}=H+v(X)$ extends the closed g-invariant 3-form $H$ if and only if the 1-forms $v(X)$ satisfy conditions (3.6).

Proof. The g-equivariance of $\hat{H}$ is the relation

$$
\mathcal{L}_{\bar{X}} \hat{H}(Y)=\mathcal{L}_{\bar{X}}(H+v(Y))=v([X, Y])
$$

that, in view of the g-invariance of $H$, reproduces the middle equality in (3.6). On the other hand, the form $\hat{H}$ is g-equivariantly closed when

$$
(\hat{d} \hat{H})(X)=d H+d v(X)-\iota_{\bar{X}} H-\iota_{\bar{X}} v(X)=0
$$

which, using that $d H=0$, is equivalent to the left and the right equalities of (3.6). 
Remark 3.8. The freedom of choice of $v(X)$ mentioned in Remark 3.2(1) consists of the addition of a 1-form $w(X)$ that is g-equivariantly closed.

The g-equivariantly closed 3-form $\hat{H}=H+v(X)$ may be related directly to the curvature of the gerbe $\mathcal{G}_{A}$ of Eq. (3.9) which is equal to the 3 -form

$$
H_{A}=H+d \rho_{A}
$$

on $\Sigma \times M$.

\section{Lemma 3.9.}

$$
H_{A}=\exp \left(-\iota_{\bar{A}}\right)(H+v(F))
$$

where $F=d A+\frac{1}{2}[A, A]$ is the gauge-field strength 2-form.

Proof. Writing $A=t^{a} A^{a}$ and $F=t^{a} F^{a}$ with $F^{a}=d A^{a}+\frac{1}{2} f^{b c a} A^{b} A^{c}$, we obtain, using the left one of relations (3.6):

$$
\begin{aligned}
H_{A} & =H+d \rho_{A}=H+d\left(-v^{a} A^{a}+\frac{1}{2}\left(\iota^{a} v^{b}\right) A^{a} A^{b}\right) \\
& =H-\iota^{a} H A^{a}+v^{a} d A^{a}+\frac{1}{2} d\left(\iota^{a} v^{b}\right) A^{a} A^{b} .
\end{aligned}
$$

Equation (3.11) permits to transform the last term on the right-hand side and to show that

$$
\begin{aligned}
H_{A} & =H-\iota^{a} H A^{a}+v^{a} d A^{a}+\frac{1}{2} f^{a b c} v^{c} A^{a} A^{b}-\frac{1}{2}\left(\iota^{a} \iota^{b} H\right) A^{a} A^{b} \\
& =H-\iota_{\bar{A}} H+v(F)+\frac{1}{2} \iota_{\bar{A}}^{2} H=\exp \left(-\iota_{\bar{A}}\right)(H+v(F)) .
\end{aligned}
$$

Remark 3.10. The minimal coupling operator $\exp \left(-\iota_{\bar{A}}\right)$ may be naturally interpreted within equivariant cohomology, see [38]. Let us only mention here that it satisfies the relation

$$
\exp \left(\iota_{\bar{A}}\right) d \exp \left(-\iota_{\bar{A}}\right)=d-\iota_{\bar{F}}+\mathcal{L}_{\bar{A}}
$$

for $\mathcal{L}_{\bar{A}}=A^{a} \mathcal{L}^{a}$

3.3. More equivariance properties. We shall assume below that the 3 -form $H$ extends to the $\Gamma$-equivariantly closed 3-form $\hat{H}(X)=H+v(X)$. This means, along with conditions (3.6), that

$$
\ell_{\gamma}^{*} H=H \text { and } \quad \ell_{\gamma}^{*} v(X)=v\left(A d_{\gamma^{-1}} X\right)
$$

for all $\gamma \in \Gamma$ and all $X \in \mathbf{g}$, see Eq. (3.15). In this section, we shall calculate the pullback $\ell^{*} H$ of the 3 -form $H$ along the action map $\ell: \Gamma \times M \rightarrow M$. The result provides another way to express equivariance properties of $H$ that will be used in the sequel.

More generally, we shall discuss below forms and gerbes over the product spaces $\Gamma^{p-1} \times M$ that will be considered as $\Gamma$-spaces with the adjoint action of $\Gamma$ on the factors 
in $\Gamma^{p-1}$ and the original one on $M$. For a sequence of indices $1 \leq i_{1}<\cdots i_{k_{1}}<i_{k_{1}+1}<$ $\cdots<i_{k_{2}}<\cdots<i_{k_{q}} \leq p$, we shall denote by $\ell_{i_{1} \ldots i_{k_{1}}, i_{k_{1}+1} \ldots i_{k_{2}}, \ldots, i_{k_{q-1}+1} \ldots i_{k_{q}}}$ the maps

$$
\begin{aligned}
& \Gamma^{p-1} \times M \ni\left(\gamma_{1}, \ldots, \gamma_{p-1}, m\right) \\
& \longmapsto\left\{\begin{array}{lll}
\left(\gamma_{i_{1}} \cdots \gamma_{i_{1}}, \gamma_{i_{k_{1}+1}} \cdots \gamma_{i_{k_{2}}}, \ldots, \gamma_{i_{k_{q-1}+1}} \cdots \gamma_{i_{k_{q}}}\right) & \in \Gamma^{q} & \text { if } i_{k_{q}}<p, \\
\left(\gamma_{i_{1}} \cdots \gamma_{i_{k_{1}}}, \gamma_{i_{k_{1}+1}} \cdots \gamma_{i_{k_{2}}}, \ldots, \gamma_{i_{k_{q-1}+1}} \cdots \gamma_{i_{k_{q}-1}} m\right) & \in \Gamma^{q-1} \times M & \text { if } i_{k_{q}}=p,
\end{array}\right.
\end{aligned}
$$

e.g., $\ell_{2}(\gamma, m)=m, \ell_{12}(\gamma, m)=\gamma m, \ell_{12}\left(\gamma_{1}, \gamma_{2}, m\right)=\gamma_{1} \gamma_{2}$, or $\ell_{2,3}\left(\gamma_{1}, \gamma_{2}, m\right)=$ $\left(\gamma_{2}, m\right)$. All these maps commute with the action of $\Gamma$. Finally, we shall abbreviate $\ell_{i_{1} \ldots i_{k} p}^{*} H:=H_{i_{1} \ldots i_{k} p}$. Similar self-explanatory shorthand notations will be employed for other forms, gerbes and gerbe 1- and 2-morphisms, also living on other product spaces.

Let us start by considering the pullback $H_{12}=\ell^{*} H$ of the 3-form $H$ to $\Gamma \times M$. The 1-forms $v(X)$ on $M$ define a 2-form

$$
\rho:=-v(\Theta)+\frac{1}{2}\left(\iota_{\bar{\Theta}} v\right)(\Theta)
$$

on $\Gamma \times M$, where $\Theta=t^{a} \Theta^{a}=\gamma^{-1} d \gamma$ is the g-valued Maurer-Cartan 1-form on $\Gamma$. As before, we use the notations $\iota_{\bar{X} \otimes \alpha}:=\alpha \iota_{\bar{X}}$ and $v(X \otimes \alpha):=v(X) \alpha$ for $X \in \mathbf{g}$ and $\alpha$ a form, dropping the exterior product sign. Note the similarity to formula (3.9) for the 2-form $\rho_{A}$.

Lemma 3.11. $H_{12}=d \rho+H_{2}$.

Proof. In order to find an explicit expression for $H_{12}$, a useful tool is the observation that, for a form $\omega \in \Omega(M)$,

$$
\left(\ell^{*} \omega\right)(\gamma, m)=\left(\exp \left[-\iota_{\bar{\Theta}(\gamma)}\right] \ell_{\gamma}^{*} \omega\right)(m) .
$$

Equation (3.27) makes explicit the contributions to $\ell^{*} \omega$ with differentials along $\Gamma$ and along $M$. Application of identity (3.27) to $\omega=H$ gives

$$
\begin{aligned}
\left(\ell^{*} H\right)(\gamma, m)= & \left(\exp \left[-\iota_{\Theta(\gamma)}\right] \ell_{\gamma}^{*} H\right)(m)=\left(\exp \left[-\iota_{\bar{\Theta}(\gamma)}\right] H\right)(m) \\
= & H(m)-\Theta^{a}(\gamma)\left(\iota^{a} H\right)(m)-\frac{1}{2}\left(\Theta^{a} \Theta^{b}\right)(\gamma)\left(\iota^{a} \iota^{b} H\right)(m) \\
& +\frac{1}{6}\left(\Theta^{a} \Theta^{b} \Theta^{c}\right)(\gamma)\left(\iota^{a} \iota^{b} \iota^{c} H\right)(m) \\
= & H(m)-\Theta^{a}(g)\left(d v^{a}\right)(m)-\frac{1}{2} f^{a b c}\left(\Theta^{a} \Theta^{b}\right)(\gamma) v^{c}(m) \\
& +\frac{1}{2}\left(\Theta^{a} \Theta^{b}\right)(\gamma)\left(d \iota^{a} v^{b}\right)(m)+\frac{1}{2} f^{b c d}\left(\Theta^{a} \Theta^{b} \Theta^{c}\right)(\gamma)\left(\iota^{a} v^{d}\right)(m) \\
= & H(m)+\left[d\left(\Theta^{a} v^{a}+\frac{1}{2} \Theta^{a} \Theta^{b} \iota^{a} v^{b}\right)\right](\gamma, m),
\end{aligned}
$$

where the last but one equality was obtained by employing relations (3.6) and Lemma 3.6, and the last equality follows from the structure equation $d \Theta^{c}=-\frac{1}{2} f^{a b c} \Theta^{a} \Theta^{b}$ for the Maurer-Cartan forms. The result is the claimed identity. 
Remark 3.12. 1. Similarly one may prove the relation

$$
\hat{H}_{12}=\hat{d} \rho+\hat{H}_{2}
$$

which gives an equivariant extension of Lemma 3.11.

2. Lemma 3.11 implies that if $w(X)$ is a 1-form depending linearly on $X$ that is $\Gamma$-equivariantly closed, then the 2 -form

$$
\sigma=-w(\Theta)+\frac{1}{2} \iota_{\bar{\Theta}} w(\Theta)
$$

on $\Gamma \times M$ is closed, see Remark 3.8. This is still true if $w(X)$ is only g-equivariantly closed.

Lemma 3.13. The 2-form $\rho$ defined in Eq. (3.26) has the following properties:

1. $\rho$ is a $\Gamma$-invariant form on $\Gamma \times M$.

2. As forms on $\Gamma^{2} \times M$,

$$
\rho_{12,3}=\rho_{1,23}+\rho_{2,3} \text {. }
$$

A proof of Lemma 3.13 may be found in Appendix 2.

\section{Global Gauge Anomalies}

4.1. General gauge transformations. As we have seen, conditions (3.6) assure the infinitesimal gauge invariance of the Feynman amplitudes (3.10). In the present section, we shall examine the behavior of the amplitudes under general gauge transformations generated by $\Gamma$-valued smooth maps $h: \Sigma \rightarrow \Gamma$. Such maps act on the space $\Sigma \times M$ by

$$
(x, m) \stackrel{L_{h}}{\longrightarrow}(x, h(x) m),
$$

on the sigma-model fields $\varphi: \Sigma \rightarrow M$ by

$$
\varphi \longmapsto h \varphi,
$$

where $(h \varphi)(x)=h(x) \varphi(x)$, and on the gauge fields according to the formulae

$$
A \longmapsto h A:=A d_{h}(A)+\left(h^{-1}\right)^{*} \Theta, \quad F \longmapsto h F=A d_{h}(F) .
$$

The infinitesimal gauge transformations are then generated by taking $h=\mathrm{e}^{-t \Lambda}$ for $\Lambda: \Sigma \rightarrow \mathbf{g}$ and expanding to the $1^{\text {st }}$ order in $t$. Let us start by establishing the transformation rule of the curvature 3 -form $H_{A}$ of gerbe $\mathcal{G}_{A}$ over $\Sigma \times M$ under maps (4.1).

Lemma 4.1. The 3-form $H_{A}$ defined in (3.19) transforms covariantly under the general gauge transformations $h: \Sigma \rightarrow \Gamma$ :

$$
L_{h}^{*} H_{A}=H_{h^{-1} A}
$$


Proof. By virtue of the formula (3.27), Lemma 3.9, the identity

$$
\iota \overline{A d_{\gamma}-1} \ell_{\gamma}^{*}=\ell_{\gamma}^{*} \iota_{\bar{X}}
$$

that holds on $M$, and relations (3.24), we have:

$$
\begin{aligned}
& \left(L_{h}^{*} H_{A}\right)(x, m)=H_{A}(x, h(x) m)=\left[\exp \left[-\iota \overline{\left(h^{*} \Theta\right)(x)}\right] \ell_{h(x)}^{*} H_{A}(x, \cdot)\right](m) \\
& =\left[\exp \left[-\iota_{\left(h^{*} \Theta\right)(x)}\right] \ell_{h(x)}^{*} \exp \left[-\iota_{\bar{A}(x)}\right](H+v(F(x)))\right](m) \\
& =\left[\exp \left[-\iota \overline{\left(h^{*} \Theta\right)(x)}\right] \exp \left[-\iota \frac{\overline{\left.\left(A d_{h^{-1}}(A)\right)(x)\right)}}{]}\left(H+v\left(\left(A d_{h^{-1}}(F)\right)(x)\right)\right)\right](m)\right. \\
& =\left[\exp \left[-\iota \frac{}{\left(h^{*} \Theta+A d_{h^{-1}}(A)\right)(x)}\right]\left(H+v\left(\left(A d_{h^{-1}}(F)\right)(x)\right)\right)\right](m) \\
& =H_{h^{-1} A}(x, m) \text {, }
\end{aligned}
$$

where the last equality follows from relations (4.3).

We shall need below a few simple facts from the theory of gerbes. First, the pullback and the tensor product of gerbes commute. Second, the pullback of the gerbe $\mathcal{I}_{B}$ associated to a 2-form $B$ is a similar gerbe associated to the pullback 2-form. Third, the tensor product of gerbes $\mathcal{I}_{B_{1}} \otimes \mathcal{I}_{B_{2}}$ for 2-forms $B_{i}$ on the same space may be identified with the gerbe $\mathcal{I}_{B_{1}+B_{2}}$. Fourth, the tensor product $\mathcal{G} \otimes \mathcal{G}^{*}$ of a gerbe with its dual is canonically isomorphic to the trivial gerbe $\mathcal{I}_{0}$ which provides the unity of the tensor product. Fifth, if two gerbes are 1-isomorphic then so are their tensor products by a third gerbe and their pullbacks by the same map.

To find out the transformation rules of the Feynman amplitudes under general gauge transformations, we have to compare the amplitudes $\boldsymbol{A}_{W Z}(h \varphi, h A)$ and $\boldsymbol{A}_{W Z}(\varphi, A)$. Since

$$
\boldsymbol{A}_{W Z}(h \varphi, h A)=\operatorname{Hol}_{\mathcal{G}_{h A}}\left(L_{h} \circ \phi\right)=\operatorname{Hol}_{L_{h}^{*} \mathcal{G}_{h A}}(\phi) \quad \text { and } \quad \boldsymbol{A}_{W Z}(\varphi, A)=\operatorname{Hol}_{\mathcal{G}_{A}}(\phi)
$$

for $\phi=(I d, \varphi)$, it will be enough to compare the gerbes $L_{h}^{*} \mathcal{G}_{h A}$ and $\mathcal{G}_{A}$ whose curvatures, equal to $L_{h}^{*} H_{h A}$ and $H_{A}$, respectively, coincide by Lemma 4.1. From the latter property, it follows that those two gerbes are related up to 1-isomorphism by tensoring with a flat gerbe which we shall identify now. Consider the gerbe

$$
\mathcal{F}=\mathcal{G}_{12} \otimes \mathcal{G}_{2}^{*} \otimes \mathcal{I}_{-\rho}
$$

over $\Gamma \times M$. It follows from Lemma 3.11 that $\mathcal{F}$ is flat.

Proposition 4.2. The gerbes $L_{h}^{*} \mathcal{G}_{h A}$ and $\mathcal{G}_{A} \otimes(h \times I d)^{*} \mathcal{F}$ over $\Sigma \times M$ are 1 -isomorphic.

A proof of Proposition 4.2 by a chain of relations, based on the properties of gerbes listed above, may be found in Appendix 3.

Taking into account relations (4.7) and the identities $\operatorname{Hol}_{(h \times I d) * \mathcal{F}}(\phi)=\operatorname{Hol}_{\mathcal{F}}((h \times$ Id $\circ \circ \phi)=\operatorname{Hol}_{\mathcal{F}}((h, \varphi))$, Proposition 4.2 implies immediately the following transformation property of the Wess-Zumino amplitudes: 
Theorem 4.3. Under the gauge transformation induced by a map $h: \Sigma \rightarrow \Gamma$,

$$
\boldsymbol{A}_{W Z}(h \varphi, h A)=\boldsymbol{A}_{W Z}(\varphi, A) \operatorname{Hol}_{\mathcal{F}}((h, \varphi)) .
$$

One can be more specific. Note that from Eq. (4.8) it follows that

$$
\operatorname{Hol}_{\mathcal{F}}((h, \varphi))=\operatorname{Hol}_{\mathcal{G}}(h \varphi) \operatorname{Hol}_{\mathcal{G}}(\varphi)^{-1} \exp \left(-i \int_{\Sigma}(h, \varphi)^{*} \rho\right) .
$$

In particular, taking $h=1$, we infer that $\operatorname{Hol}_{\mathcal{F}}((1, \varphi))=1$. Indeed, the 2-form $(1, \varphi)^{*} \rho$ on $\Sigma$ vanishes because the 2 -form $\rho$ is composed of terms of degree $\leq 1$ in the direction of $M$. More generally, since the flat-gerbe holonomies of homotopic fields coincide by virtue of the holonomy property $(2.4), \operatorname{Hol}_{\mathcal{F}}((h, \varphi))=1$ if $h$ is homotopic to 1 .

Corollary 4.4. The Feynman amplitudes (3.10) are invariant under gauge transformations homotopic to 1 .

The gauge transformations homotopic to 1 are often called small. The remaining issue is the invariance of the amplitudes (3.10) under large gauge transformations that are not homotopic to 1 . The holonomy of the flat gerbe $\mathcal{F}$ on $\Gamma \times M$ defines a cohomology class $[\mathcal{F}] \in H^{2}(\Gamma \times M, U(1))$ which is trivial if and only if the flat gerbe $\mathcal{F}$ is 1 -isomorphic to the trivial gerbe $\mathcal{I}_{0}$. By virtue of definition (4.8), the latter holds if and only if the gerbes $\mathcal{G}_{12}$ and $\mathcal{I}_{\rho} \otimes \mathcal{G}_{2}$ over $\Gamma \times M$ are 1-isomorphic. Consequently,

Corollary 4.5. The amplitudes (3.10) are invariant under all gauge transformations if and only if the gerbes $\mathcal{G}_{12}$ and $\mathcal{I}_{\rho} \otimes \mathcal{G}_{2}$ over $\Gamma \times M$ are 1-isomorphic.

The class $[\mathcal{F}]$, that will be more carefully studied in Sect. 6 , is the obstruction to the invariance of the Feynman amplitudes (3.10) under large gauge transformations. In other words, a non-triviality of the class $[\mathcal{F}]$ leads to a global gauge anomaly in the two-dimensional sigma model with the Wess Zumino term corresponding to the gerbe $\mathcal{G}$ and coupled to topologically trivial gauge fields.

In the above analysis, we kept fixed the $\Gamma$-equivariant extension $\hat{H}+v(X)$ of the curvature $H$ of the gerbe $\mathcal{G}$. A natural question arises whether one may use the freedom in the choice of $v(X)$ to annihilate the global gauge anomaly. Clearly, the answer is that this may be done if and only if there exists a 1-form $w(X)$ that is $\Gamma$-equivariantly closed for which $[\mathcal{F}]=[\sigma]$, where $[\sigma]$ denotes the cohomology class in $H^{2}(\Gamma \times M, U(1))$ induced by the closed 2-form $\sigma$ of Eq. (3.30). In many contexts, however, e.g., in applications to WZW and coset models of conformal field theory, that we shall discuss below, $v(X)$ is a part of the structure tied to the symmetries of the theories and should not be changed.

Similarly, one may ask whether it is possible to annihilate the global gauge anomaly by an appropriate choice of gerbe $\mathcal{G}$, keeping the curvature form fixed. Since this involves tensoring $\mathcal{G}$ with flat gerbes whose 1-isomorphism classes belong to $H^{2}(M, U(1))$, the answer is that this is possible if and only if $[\mathcal{F}]=[b]_{12}-[b]_{2}$ for some class $[b] \in H^{2}(M, U(1))$. A change of $\mathcal{G}$ to another non 1-isomorphic gerbe, however, implies a non-trivial change of the Feynman amplitudes of the ungauged sigma model, i.e. of the model itself. 
4.2. Global gauge anomalies in WZW amplitudes. As an example, let us consider the case when $M=G$, where $G$ is a connected compact semi-simple Lie group, not necessarily simply connected. One has: $G=\tilde{G} / Z$, where $\tilde{G}=\times_{l} \tilde{G}_{l}$ is the covering group of $G$ that decomposes into the product of simple factors, and $Z$ is a subgroup of the center $\tilde{Z}=\times_{l} \tilde{Z}_{l}$ of $\tilde{G}$. The factors $\tilde{Z}_{l}$ are cyclic except for those equal to $\mathbb{Z}_{2}^{2}$ corresponding to $\tilde{G}_{l}=\operatorname{Spin}(4 r)$. The Lie algebra $\mathbf{g}$ of $\tilde{G}$ decomposes as $\oplus_{l} \mathbf{g}_{l}$ into the direct sum of simple factors. Let $\mathbf{h}$ be a Lie subalgebra of $\mathbf{g}$ corresponding to a connected but not necessarily simply connected closed subgroup $\tilde{H} \subset \tilde{G}$ that maps onto a closed connected subgroup $\Gamma$ of $\tilde{G} / \tilde{Z}$. Clearly, $\mathbf{h}$ is also the Lie algebra of $\Gamma$ and $\Gamma=\tilde{H} / Z_{\Gamma}$ with $Z_{\Gamma}=\tilde{H} \cap \tilde{Z}$. We shall consider $G$ with the adjoint action of $\Gamma$.

Definition 4.6. Below, we shall call a $\Gamma$-space $M=G$ as above the one of the cosetmodel context.

In the simplest case, $\mathbf{h}=\mathbf{g}$ and $\Gamma=\tilde{G} / \tilde{Z}$. In what follows, the reader may think about this example.

Over the group $G=\tilde{G} / Z$, we shall consider gerbes $\mathcal{G}_{k}$ with the curvature 3-forms

$$
H_{k}=\frac{1}{12 \pi} k \operatorname{tr} \Theta^{3},
$$

where $\Theta=g^{-1} d g$ is the g-valued Maurer-Cartan 1-form on $G$ and $k \operatorname{tr} X Y:=$ $\sum k_{l} \operatorname{tr}_{l} X^{l} Y^{l}$ stands for the $a d$-invariant negative-definite bilinear form on $\mathbf{g}$ given by the sum of such forms on $\mathbf{g}_{l}$. We assume that the latter are normalized so that, if $G=\tilde{G}$, then the form $H_{k}$ has periods in $2 \pi \mathbb{Z}$ if and only if the level $k=\left(k_{l}\right)$ is composed of integers. For non-simply connected groups $G, k$ has to satisfy more stringent selection rules to assure the integrality of periods of $\frac{1}{2 \pi} H[15,24,41]$. The holonomy of gerbes $\mathcal{G}_{k}$ provides the Wess-Zumino part of amplitudes for the WZW sigma models of conformal field theory [59], see the next section.

Definition 4.7. We shall call $\mathcal{G}_{k}$ a WZW gerbe.

There may be several non-1-isomorphic WZW gerbes $\mathcal{G}_{k}$ over $G$ (their 1-isomorphism classes are counted by elements of $H^{2}(Z, U(1))$ in the discrete group $Z$ cohomology [4]). The adjoint action of group $\tilde{G} / \tilde{Z}$ leaves the 3 -forms $H_{k}$ invariant. For $X \in \mathbf{g}$, the vector field $\bar{X}$ on $G$ induced by the infinitesimal adjoint action: $\bar{X}(g)=\left.\frac{d}{d t}\right|_{t=0} A d_{\mathrm{e}^{-t X}}(g)$ satisfies the relation $\iota_{\bar{X}} \Theta(g)=X-A d_{g^{-1}}(X)$. Hence,

$$
\iota_{\bar{X}} H_{k}=\frac{1}{8 \pi} k \operatorname{tr} X\left(1-A d_{g}\right)([\Theta(g), \Theta(g)])=-\frac{1}{4 \pi} d k \operatorname{tr} X\left(1+A d_{g}\right)(\Theta(g))
$$

so that, upon setting

$$
v_{k}(X)=-\frac{1}{4 \pi} k \operatorname{tr} X\left(1+A d_{g}\right)(\Theta(g)),
$$

the left one of conditions (3.6) is satisfied. The 1-forms $v_{k}(X)$ satisfy also the other conditions of (3.6). Indeed,

$$
\begin{aligned}
\iota_{\bar{X}} v_{k}(Y) & =-\frac{1}{4 \pi} k \operatorname{tr} Y\left(-A d_{g-1}(X)+A d_{g}(X)\right) \\
& =\frac{1}{4 \pi} \operatorname{tr} X\left(-A d_{g-1}(Y)+A d_{g}(Y)\right)=-\iota_{\bar{Y}} v_{k}(X),
\end{aligned}
$$




$$
\begin{aligned}
\mathcal{L}_{\bar{X}} v_{k}(Y) & =-\left.\frac{d}{d t}\right|_{t=0} \frac{1}{4 \pi} k \operatorname{tr} Y A d_{\mathrm{e}^{-t X}}^{*}\left(\left(1+A d_{g}\right)(\Theta(g))\right) \\
& =-\left.\frac{d}{d t}\right|_{t=0} \frac{1}{4 \pi} k \operatorname{tr} Y A d_{\mathrm{e}^{-t X}}\left(\left(1+A d_{g}\right)(\Theta(g))\right) \\
& =-\left.\frac{d}{d t}\right|_{t=0} \frac{1}{4 \pi} k \operatorname{tr} A d_{\mathrm{e}^{t X}}(Y)\left(1+A d_{g}\right)(\Theta(g))=v_{k}([X, Y]) .
\end{aligned}
$$

Of course, we may restrict $X, Y$ above to take values in the subalgebra $\mathbf{h} \subset \mathbf{g}$. The 2-form $\rho_{k, A}$ on $\Sigma \times \Gamma$ defined by Eq. (3.9) and the 2-form $\rho_{k}$ on $\Gamma \times G$ defined by Eq. (3.26) are given now by the formulae

$$
\begin{aligned}
\rho_{k, A} & =\frac{1}{4 \pi} k \operatorname{tr}\left(\left(1+A d_{g}\right)(\Theta(g))+A d_{g^{-1}}(A)\right) A, \\
\rho_{k} & =\frac{1}{4 \pi} k \operatorname{tr}\left(\left(1+A d_{g}\right)(\Theta(g))+A d_{g^{-1}}(\Theta(\gamma))\right) \Theta(\gamma),
\end{aligned}
$$

where $\Theta(\gamma)=\gamma^{-1} d \gamma$ is the Maurer-Cartan form on $\Gamma$. The 2-form $\rho_{k, A}$ enters the coupling, described in Definition 3.3, of the Wess-Zumino action to the h-valued 1-form $A$ on $\Sigma$.

Let us compute the holonomy of the flat gerbe $\mathcal{F}_{k}=\left(\mathcal{G}_{k}\right)_{12} \otimes\left(\mathcal{G}_{k}\right)_{2}^{*} \otimes \mathcal{I}_{-\rho_{k}}$ over $\Gamma \times G$, see Eq. (4.8). Recall that the non-triviality of such holonomy obstructs the invariance of the Wess-Zumino amplitudes of Definition 3.3 under large gauge transformations. By Eq. (4.10), for $h: \Sigma \rightarrow \Gamma$ and $\varphi: \Sigma \rightarrow G$,

$$
\operatorname{Hol}_{\mathcal{F}_{k}}((h, \varphi))=\operatorname{Hol}_{\mathcal{G}_{k}}\left(\operatorname{Ad}_{h}(\varphi)\right) \operatorname{Hol}_{\mathcal{G}_{k}}(\varphi)^{-1} \exp \left(-i \int_{\Sigma}(h, \varphi)^{*} \rho_{k}\right)=: c_{h, \varphi} .
$$

Since $\mathcal{F}_{k}$ is flat, the above holonomy depends only on the homotopy classes $[h]$ and $[\varphi]$ of the maps $h$ and $\varphi$. Besides it does not depend on whether we treat $h$ as a map with values in $\Gamma$ or in $\tilde{G} / \tilde{Z}$. In the latter case, the homotopy classes of the maps $h$ are in one-to-one relation with the elements of $Z_{\Gamma}^{2 \omega}$, where $\omega$ is the genus of $\Sigma$. The element $\left(\tilde{z}_{1}, \tilde{z}_{2}, \ldots, \tilde{z}_{2 \omega-1}, \tilde{z}_{2 \omega}\right)$ corresponding to $[h]$ is given by the windings of $h$ described by the holonomies

$$
\tilde{z}_{2 j-1}=\mathcal{P} \exp \left(\int_{a_{j}} h^{*} \Theta\right), \quad \tilde{z}_{2 j}=\mathcal{P} \exp \left(\int_{b_{j}} h^{*} \Theta\right)
$$

of the non-Abelian flat gauge field $h^{*}(\Theta)$ on $\Sigma$. Above, $\mathcal{P}$ stands for the path-ordering (from left to right) along paths $a_{j}, b_{j}, j=1, \ldots, \omega$, that generate a fixed marking of the surface $\Sigma$, the latter assumed here to be connected, see Fig. 1. Similarly for elements $\left(z_{1}, \ldots, z_{2} \omega\right)$ describing the windings of $\varphi$ belonging to $Z^{2 \omega}$. By pinching off the handles of the surface the same way as in Sec. III of [25], one notes, using the commutativity of the fundamental groups of $\tilde{G} / \tilde{Z}$ and of $G$, that

$$
c_{h, \varphi} \equiv c_{\left(\tilde{z}_{1}, \ldots, \tilde{z}_{2 \omega}\right),\left(z_{1}, \ldots, z_{2 \omega}\right)}=\prod_{j=1}^{\omega} c_{\left(\tilde{z}_{2 j-1}, \tilde{z}_{2 j}\right),\left(z_{2 j-1}, z_{2 j}\right)} .
$$




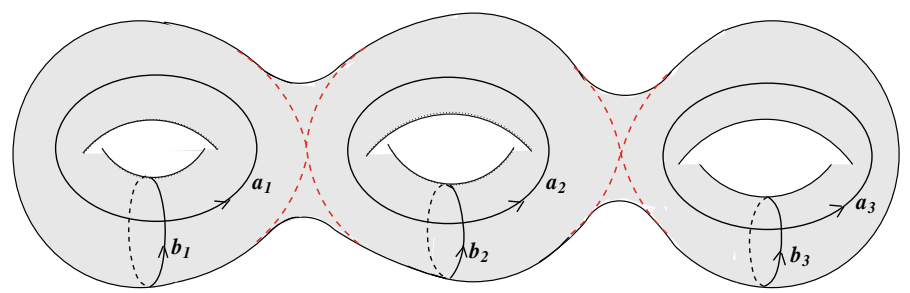

Fig. 1. Genus 3 surface with a marking; crossed broken lines (red online) indicate the contours of its version with pinched handles

Hence, the calculation of $c_{h, \varphi}$ reduces to the genus 1 case with $\Sigma=S^{1} \times S^{1}$. Let us choose the Cartan subalgebras $\mathbf{t}_{\mathbf{h}} \subset \mathbf{h}$ and $\mathbf{t}_{\mathbf{g}} \subset \mathbf{g}$ so that $\mathbf{t}_{\mathbf{h}} \subset \mathbf{t}_{\mathbf{g}}$. On $\Sigma=S^{1} \times S^{1}$, one may take $h=h_{\tilde{p}_{1}^{\vee}, \tilde{p}_{2}^{\vee}}$ and $\varphi=\varphi_{p_{1}^{\vee} \cdot p_{2}^{\vee}}$ with

$$
h_{\tilde{p}_{1}^{\vee}, \tilde{p}_{2}^{\vee}}\left(e^{i \sigma_{1}}, \mathrm{e}^{i \sigma_{2}}\right)=\mathrm{e}^{i\left(\sigma_{1} \tilde{p}_{1}^{\vee}+\sigma_{2} \tilde{p}_{2}^{\vee}\right)}, \quad \varphi_{p_{1}^{\vee}, p_{2}^{\vee}}\left(e^{i \sigma_{1}}, \mathrm{e}^{i \sigma_{2}}\right)=\mathrm{e}^{i\left(\sigma_{1} p_{1}^{\vee}+\sigma_{2} p_{2}^{\vee}\right)},
$$

where $\tilde{p}_{i}^{\vee} \in i \mathbf{t}_{\mathbf{h}}$ and $p_{i}^{\vee} \in i \mathbf{t}_{\mathbf{g}}$ are such that the windings $\tilde{z}_{i}=\mathrm{e}^{2 \pi i \tilde{p}_{i}^{\vee}} \in Z_{\Gamma}$ and $z_{i}=\mathrm{e}^{2 \pi i p_{i}^{\vee}} \in Z$. Note that $\tilde{p}_{i}^{\vee}$ and $p_{i}^{\vee}$ have to belong to the coweight lattice $P_{\mathbf{g}}^{\vee}$ composed of elements $p^{\vee} \in i \mathbf{t}_{\mathbf{g}}$ such that $\mathrm{e}^{2 \pi i p^{\vee}} \in \tilde{Z}$. Since $A d_{\tilde{p}_{\tilde{p}_{1}}^{\vee} \tilde{p}_{2}^{\vee}}\left(\varphi_{p_{1}^{\vee}, p_{2}^{\vee}}\right)=\varphi_{p_{1}^{\vee}, p_{2}^{\vee}}$, the formula (4.18) gives

$$
\begin{aligned}
c_{\left(\tilde{z}_{1}, \tilde{z}_{2}\right),\left(z_{1}, z_{2}\right)} & =\exp \left(-i \int_{S^{1} \times S^{1}}\left(h_{\tilde{p}_{1}^{\vee}, \tilde{p}_{2}^{\vee}}, \varphi_{p_{1}^{\vee}, p_{2}^{\vee}}\right)^{*} \rho_{k}\right) \\
& =\exp \left(\frac{i}{2 \pi} \int_{0}^{2 \pi} \int_{0}^{2 \pi} k \operatorname{tr}\left(d \sigma_{1} p_{1}^{\vee}+d \sigma_{2} p_{2}^{\vee}\right)\left(d \sigma_{1} \tilde{p}_{1}^{\vee}+d \sigma_{2} \tilde{p}_{2}^{\vee}\right)\right) \\
& =\exp \left(2 \pi i k \operatorname{tr}\left(p_{1}^{\vee} \tilde{p}_{2}^{\vee}-\tilde{p}_{1}^{\vee} p_{2}^{\vee}\right)\right) .
\end{aligned}
$$

That the right hand side depends only on the windings is assured by the integrality of the level $k$. The holonomy of the flat gerbe $\mathcal{F}_{k}$ is trivial if and only if the above expression is always equal to 1 for the windings restricted as above (compare to a similar discussion in [25]). From Corollary 4.3, we obtain

Proposition 4.8. For the $\Gamma$-space $M=G$ in the coset-model context, see Definition 4.6, the WZ Feynman amplitudes (3.10) are invariant under all gauge transformations if and only if the phases (4.22) are trivial.

When $G=\tilde{G}$, one may take $p_{i}^{\vee}=0$ so that the phases (4.22) are trivial. We obtain this way

Corollary 4.9. For the simply connected $\Gamma$-space $M=\tilde{G}$ in the coset-model context, the WZ Feynman amplitudes (3.10) are invariant under all gauge transformations.

For non-simply connected groups $G=\tilde{G} / Z$, examples where the phases (4.22) are non-trivial are numerous. They include $G=\Gamma=\tilde{G} / \tilde{Z}$ for $\tilde{G}=S U(r+1)$ with $r$ even and $k=1$ or with $r \geq 3$ odd and $k=2$. Another example is $G=\Gamma=\operatorname{Spin}(2 r) / \mathbb{Z}_{2}^{2}$ with $r$ divisible by 4 and $k=1$. In all those cases (and many others), the amplitudes (3.10) of Definition 3.3 exhibit a global gauge anomaly. 
The best known case with a non-simple group $\tilde{G}$ is $\tilde{G}=S U(2) \times S U(2)$. The restrictions on the level $k=\left(k_{1}, k_{2}\right)$ imposed by the existence of the gerbe $\mathcal{G}_{k}$ with curvature $H_{k}$ on $G=\tilde{G} / Z$ depend on $Z \subset \tilde{Z}=\mathbb{Z}_{2} \times \mathbb{Z}_{2}$.

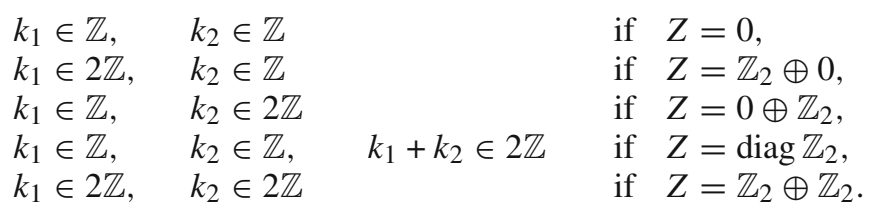

For $\Gamma=\tilde{G} / \tilde{Z}=S O(3) \times S O(3)$, with the adjoint action on $G$ and $\tilde{p}_{i}, \tilde{p}_{i}^{\prime}, p_{i}, p_{i}^{\prime} \in \mathbb{Z}$,

$$
\begin{aligned}
& c_{\left(\left((-1) \tilde{p}_{1,(-1)} \tilde{p}_{1),((-1)}^{\prime} \tilde{p}_{2,(-1)} \tilde{p}_{2}^{\prime}\right)\right),\left(\left((-1)^{p_{1,(-1)}} p_{1),((-1)}^{\prime} p_{2,(-1)} p_{2))}^{\prime}\right.\right.} \\
& \quad=(-1)^{k_{1}\left(p_{1} \tilde{p}_{2}-\tilde{p}_{1} p_{2}\right)+k_{2}\left(p_{1}^{\prime} \tilde{p}_{2}^{\prime}-\tilde{p}_{1}^{\prime} p_{2}^{\prime}\right)} .
\end{aligned}
$$

We infer from this expression that the only case with a global anomaly of the gauged WZ amplitudes (3.10) of Definition 3.3 is the one with $G=(S U(2) \times S U(2)) / \operatorname{diag} \mathbb{Z}_{2}$ with odd $k_{1}, k_{2}$. If one restricts, however, the group $\Gamma$ to the diagonal $S O(3)$ subgroup of $S O(3) \times S O(3)$ then the global gauge anomaly disappears. Another anomalous example with a non-simple group is $G=(S U(3) \times S U(3)) /\left(\mathbb{Z}_{3} \times \mathbb{Z}_{3}\right)$ at level $k=(1,1)$ with the adjoint action of $\Gamma=\operatorname{diag}\left(S U(3) / \mathbb{Z}_{3}\right)$.

The non-anomalous gauging of the adjoint action of the diagonal $S O$ (3) subgroup in the WZW model with groups $(S U(2) \times S U(2)) / Z$ is used in the coset model construction [30] of the unitary minimal models of conformal field theory $[18,22,33]$. Other coset theories involve other versions of gauged WZW amplitudes and may suffer from global anomalies, as will be discussed below.

4.3. Anomalies and WZW partition functions. The results of the calculation of the global-gauge-anomaly phases in the last section are consistent with the exact solution for the toroidal partition functions of the WZW models of conformal field theory in an external gauge field.

Let us start by considering the level $k$ WZW sigma model on a closed Riemann surface $\Sigma$ with the Lie group $G=\tilde{G} / Z$ as the target manifold. The Feynman amplitude of a field $\varphi: \Sigma \rightarrow G$ in the background of the external gauge field described by a g-valued 1-form $A$ on $\Sigma$ is given by the formula

$$
\boldsymbol{A}_{W Z W}(\varphi, A)=\exp \left(\frac{i}{4 \pi} \int_{\Sigma} k \operatorname{tr}\left(\varphi^{-1} \partial_{A} \varphi\right)\left(\varphi^{-1} \bar{\partial}_{A} \varphi\right)\right) \mathcal{A}_{W Z}(\varphi, A),
$$

where $\partial_{A}=\partial+a d_{A^{10}}$ and $\bar{\partial}_{A}=\bar{\partial}+\operatorname{ad}_{A^{01}}$ are the minimally coupled Dolbeault differentials relative to the complex structure of $\Sigma$, for $A=A^{10}+A^{01}$. The WZ amplitude $\boldsymbol{A}_{W Z}(\varphi, A)$ is related to the holonomy of the WZW gerbe $\mathcal{G}_{k}$ on $G$, with the adjoint action of the group $\Gamma=\tilde{G} / \tilde{Z}$ gauged as described previously.

Let $\Sigma=\mathcal{T}_{\tau}:=\mathbf{C} /(2 \pi \mathbb{Z}+2 \pi \tau \mathbb{Z})$ be the complex torus with the modular parameter $\tau=\tau_{1}+i \tau_{2}$, where the imaginary part $\tau_{2}>0$. The toroidal partition function is formally defined by the functional integral over the space of maps $\varphi: \mathcal{T}_{\tau} \rightarrow G$

$$
\mathcal{Z}^{G}(\tau, A)=\int_{M a p\left(\mathcal{T}_{\tau}, G\right)} \boldsymbol{A}_{W Z W}(\varphi, A) D \varphi .
$$


Its exact form may be found from (formal) symmetry properties of the functional integral. The result has a specially simple form for the gauge fields

$$
A_{u}=\frac{\bar{u} d w-u d \bar{w}}{2 \tau_{2}}
$$

with $u$ in the complexified Cartan algebra $\mathbf{t}_{\mathbf{g}}^{\mathbf{C}}$ of $\mathbf{g}$ and $w$ the coordinate on the complex plane (for other gauge fields, it is then determined by chiral gauge transformations [19]). When the group $G$ is simply connected, i.e. $G=\tilde{G}$, one has

$$
\mathcal{Z}^{\tilde{G}}\left(\tau, A_{u}\right)=\sum_{\Lambda \in P_{k}^{+}(\mathbf{g})}\left|\chi_{k, \Lambda}^{\hat{\mathbf{g}}}(\tau, u)\right|^{2} \exp \left[\frac{\pi k \operatorname{tr}(u-\bar{u})^{2}}{2 \tau_{2}}\right],
$$

where $\chi_{k, \Lambda}^{\hat{\mathbf{g}}}$ are the affine characters,

$$
\chi_{k, \Lambda}^{\hat{\mathbf{g}}}(\tau, u)=\operatorname{tr}_{V_{k, \Lambda}^{\hat{\mathbf{g}}}} \exp \left(2 \pi i\left[\tau\left(L_{0}^{\hat{\mathbf{g}}}-\frac{c_{k}^{\hat{\mathbf{g}}}}{24}\right)+u\right]\right),
$$

of the unitary highest-weight modules $V_{k, \Lambda}^{\hat{\mathbf{g}}}$ of level $k$ and highest weight $\Lambda$ of the affine algebra $\hat{\mathbf{g}}$ associated to the Lie algebra $\mathbf{g}[28,39] . L_{0}^{\hat{\mathbf{g}}}$ stands for the corresponding Sugawara-Virasoro generator and $c_{k}^{\hat{\mathbf{g}}}$ for the Virasoro central charge. The admissible highest weights $\Lambda$ form a finite set $P_{k}^{+}(\mathbf{g})$. We consider weights as elements of $i \mathbf{t}_{\mathbf{g}}$, identifying the latter space with its dual by means of the bilinear form tr.

For non-simply connected groups $G=\tilde{G} / Z$, the toroidal partition functions take a more complicated form [15]. The space of (regular) maps from $\mathcal{T}_{\tau}$ to $G$ has different connected components that may be labeled by the windings:

$$
\operatorname{Map}\left(\mathcal{T}_{\tau}, G\right)=\underset{\left(z_{1}, z_{2}\right) \in Z^{2}}{\sqcup} \operatorname{Map} p_{z_{1}, z_{2}}\left(\mathcal{T}_{\tau}, \tilde{G}\right),
$$

where for $z_{i}=\mathrm{e}^{2 \pi i p_{i}^{\vee}}, \operatorname{Map}_{z_{1}, z_{2}}$ contains the maps homotopic to $\varphi_{p_{1}^{\vee}, p_{2}^{\vee}}$ of Eq. (4.21) (viewed as a map on $\mathcal{T}_{\tau}$ via the parametrization of the complex plane by $w=\sigma_{1}+\tau \sigma_{2}$ ). Let

$$
\mathcal{Z}_{z_{1}, z_{2}}^{G}(\tau, A)=\int_{\operatorname{Map}_{z_{1}, z_{2}}\left(\mathcal{T}_{\tau}, G\right)} \boldsymbol{A}_{W Z W}(\varphi, A) D \varphi
$$

so that

$$
\mathcal{Z}^{G}(\tau, A)=\sum_{\left(z_{1}, z_{2}\right) \in Z^{2}} \mathcal{Z}_{z_{1}, z_{2}}^{G}(\tau, A) .
$$

By writing $\varphi=\varphi_{p_{1}^{\vee}}, p_{2}^{\vee} \tilde{\varphi}$, where $\tilde{\varphi}$ has trivial windings and may be lifted to a map from $\mathcal{T}_{\tau}$ to $\tilde{G}$, one may relate the functional integral for $\mathcal{Z}_{z_{1}, z_{2}}^{G}(\tau, A)$ to the one for $Z^{\tilde{G}}(\tau, A)$ using the chiral Ward identities [19]. One obtains this way the formula

$$
\begin{aligned}
\mathcal{Z}_{z_{1}, z_{2}}^{G}\left(\tau, A_{u}\right)= & \frac{1}{|Z|} \sum_{\Lambda \in P_{k}^{+}(\mathbf{g})} \operatorname{Hol}_{\mathcal{G}_{k}}\left(\varphi_{p_{1}^{\vee}, p_{2}^{\vee}}\right) \exp \left[-i k \operatorname{tr} p_{1}^{\vee}\left(p_{2}^{\vee}-\tau p_{1}^{\vee}\right)-2 \pi i k \operatorname{tr} u p_{1}^{\vee}\right] \\
& \cdot \chi_{k, \Lambda}^{\hat{\mathbf{g}}}\left(\tau, u+p_{2}^{\vee}-\tau p_{1}^{\vee}\right) \overline{\chi_{k, \Lambda}^{\hat{\mathbf{g}}}(\tau, u)} \exp \left[\frac{\pi k \operatorname{tr}(u-\bar{u})^{2}}{2 \tau_{2}}\right],
\end{aligned}
$$


where $|Z|$ stands for the cardinality of $Z$ and the values of $\operatorname{Hol}_{\mathcal{G}_{k}}\left(\varphi_{p_{1}}, p_{2}^{\vee}\right)$ may be found in Sec. IV of [25]. There exists a spectral flow $\Lambda \mapsto z \Lambda$ on $P_{k}^{+}$(g) (and on the set of the corresponding highest-weight modules of $\hat{\mathbf{g}}$ ) induced by the elements $z$ of the center $\tilde{Z}$ of $\tilde{G}[15]$. The highest weight $z \Lambda$ is uniquely fixed by the property that

$$
\mathrm{e}^{2 \pi i k^{-1} z \Lambda}=z A d_{w_{z}}\left(\mathrm{e}^{2 \pi i k^{-1} \Lambda}\right)
$$

for some $w_{z}$ in the normalizer of the Cartan subgroup of $\tilde{G}$. The characters of the $\hat{\mathbf{g}}$-modules with the highest weights connected by the spectral flow satisfy the relation

$$
\begin{aligned}
& \exp \left[-\pi i k \operatorname{tr}\left(p_{2}^{\vee}-\tau p_{1}^{\vee}\right) p_{1}^{\vee}-2 \pi i k \operatorname{tr} u p_{1}^{\vee}\right] \chi_{k, \Lambda}^{\hat{\mathbf{g}}}\left(\tau, u+p_{2}^{\vee}-\tau p_{1}^{\vee}\right) \\
& \quad=\exp \left[2 \pi i \operatorname{tr} p_{2}^{\vee} \Lambda-\pi i k \operatorname{tr} p_{1}^{\vee} p_{2}^{\vee}\right] \chi_{k, z_{1}^{-1} \Lambda}^{\mathbf{g}}(\tau, u)
\end{aligned}
$$

for any $p_{1}^{\vee}$ and $p_{2}^{\vee}$ in the coweight lattice $P_{\mathbf{g}}^{\vee}$. As a result, Eq. (4.32) may be rewritten in the form

$$
\begin{aligned}
& \mathcal{Z}_{z_{1}, z_{2}}^{G}\left(\tau, A_{u}\right) \\
& \quad=\frac{1}{|Z|} \sum_{\Lambda \in P_{k}^{+}(\mathbf{g})} \epsilon_{z_{1}, z_{2}}(\Lambda) \chi_{k, z_{1}^{-1} \Lambda}^{\hat{\mathbf{g}}}(\tau, u) \overline{\chi_{k, \Lambda}^{\hat{\mathbf{g}}}(\tau, u)} \exp \left[\frac{\pi k \operatorname{tr}(u-\bar{u})^{2}}{2 \tau_{2}}\right],
\end{aligned}
$$

where

$$
\epsilon_{z_{1}, z_{2}}(\Lambda)=\operatorname{Hol}_{\mathcal{G}_{k}}\left(\varphi_{p_{1}^{\vee}, p_{2}^{\vee}}\right) \exp \left[2 \pi i \operatorname{tr} p_{2}^{\vee} \Lambda-\pi i k \operatorname{tr} p_{1}^{\vee} p_{2}^{\vee}\right]
$$

defines a character on $Z$ through its dependence on $z_{2}$. Let, for $z \in Z$,

$$
C_{z}:=\left\{\Lambda \in P_{k}^{+}(\mathbf{g}) \mid \epsilon_{z, z_{2}}(\Lambda)=1 \text { for all } z_{2} \in Z\right\} .
$$

Summing both sides of Eq. (4.35) over $z_{1}$ and $z_{2}$, one obtains the following formula for the complete partition function of the group $G$ WZW model at level $k$ :

$$
\mathcal{Z}^{G}\left(\tau, A_{u}\right)=\sum_{z \in Z} \sum_{\Lambda \in P_{k}^{+}(\mathbf{g}) \cap C_{z}} \chi_{k, z^{-1} \Lambda}^{\hat{\mathbf{g}}}(\tau, u) \overline{\chi_{k, \Lambda}^{\hat{\mathbf{g}}}(\tau, u)} \exp \left[\frac{\pi k \operatorname{tr}(u-\bar{u})^{2}}{2 \tau_{2}}\right] .
$$

Note that, for non-trivial $Z$, the affine characters and their complex conjugates are combined non-diagonally in the latter expression, in contrast with the formula (4.27). The space of states of the model that can be read off from Eq. (4.38) has the form $[15,41]$

$$
\mathbb{H}^{G}=\underset{z \in Z}{\oplus}\left(\underset{\Lambda \in P_{k}^{+}(\mathbf{g}) \cap C_{z}}{\oplus} V_{k, z^{-1} \Lambda}^{\hat{\mathbf{g}}} \otimes \overline{V_{k, \Lambda}^{\hat{\mathbf{g}}}}\right) .
$$

The transformation properties of the WZW partition function (4.35) under large gauge transformations $h_{\tilde{p}_{1}^{\vee}, \tilde{p}_{2}^{\vee}}$ of Eq. (4.21) are determined by the equality

$$
h_{\tilde{p}_{1}^{\vee}, \tilde{p}_{2}^{\vee}} A_{u}=A_{u-\tilde{p}_{2}^{\vee}+\tau \tilde{p}_{1}^{\vee}}
$$


and by identity (4.34) for the affine characters. With the help of these relations, one obtains

$$
\begin{aligned}
& \mathcal{Z}_{z_{1}, z_{2}}^{G}\left(\tau, h_{\tilde{p}_{1}^{\vee}, \tilde{p}_{2}^{\vee}} A_{u}\right)=\mathcal{Z}_{z_{1}, z_{2}}^{G}\left(\tau, A_{\left.u-\tilde{p}_{2}^{\vee}+\tau \tilde{p}_{1}^{\vee}\right)}\right. \\
& =\frac{1}{|Z|} \sum_{\Lambda \in P_{k}^{+}(\mathbf{g})} \epsilon_{z_{1}, z_{2}}(\Lambda) \exp \left[-2 \pi i \operatorname{tr} \tilde{p}_{2}^{\vee}\left(z_{1}^{-1} \Lambda-\Lambda\right)\right] \\
& \cdot \chi_{k, \tilde{z}_{1} z_{1}^{-1} \Lambda}^{\hat{\mathbf{g}}}(\tau, u) \overline{\chi_{k, \tilde{z}_{1} \Lambda}^{\hat{\mathbf{g}}}(\tau, u)} \exp \left[\frac{\pi k \operatorname{tr}(u-\bar{u})^{2}}{2 \tau_{2}}\right],
\end{aligned}
$$

where, as before, $\tilde{z}_{i}=\mathrm{e}^{2 \pi i \tilde{p}_{i}^{\vee}}$. It is easy to see, using Eq. (4.33), that

$$
\exp \left[-2 \pi i \operatorname{tr} \tilde{p}_{2}^{\vee}\left(\tilde{z}_{1}^{-1} \Lambda-\Lambda\right)\right]=\exp \left(2 \pi i k \operatorname{tr} \tilde{p}_{1}^{\vee} \tilde{p}_{2}^{\vee}\right)
$$

for any $\Lambda \in P_{k}^{+}(\mathbf{g})$. Replacing $\Lambda$ by $\tilde{z}_{1}^{-1} \Lambda$ on the right-hand side of Eq. (4.41) and using the relation

$$
\epsilon_{z_{1}, z_{2}}\left(\tilde{z}_{1}^{-1} \Lambda\right)=\exp \left[-2 \pi i k \operatorname{tr} \tilde{p}_{1}^{\vee} p_{2}^{\vee}\right] \epsilon_{z_{1}, z_{2}}(\Lambda)
$$

that follows from Eq. (4.42), one obtains

Proposition 4.10. The transformation law of the toroidal partition function (4.35) under large gauge transformations is described by the identity

$$
\mathcal{Z}_{z_{1}, z_{2}}^{G}\left(\tau, h_{\tilde{p}_{1}^{\vee}, \tilde{p}_{2}^{\vee}} A_{u}\right)=c_{\left(\tilde{z}_{1}, \tilde{z}_{2}\right),\left(z_{1}, z_{2}\right)} \mathcal{Z}_{z_{1}, z_{2}}^{G}\left(\tau, A_{u}\right),
$$

where the phases $c_{\left(\tilde{z}_{1}, \tilde{z}_{2}\right),\left(z_{1}, z_{2}\right)}$ are given by Eq. (4.22).

If we assume the gauge invariance $D \varphi=D(h \varphi)$ of the formal functional integral measure, then the above anomalous transformation property follows from the functional integral expression (4.30) and the relation

$$
\boldsymbol{A}_{W Z W}\left(h_{\tilde{p}_{1}^{\vee}, \tilde{p}_{2}^{\vee}} \varphi, h_{\tilde{p}_{1}^{\vee}, \tilde{p}_{2}^{\vee}} A\right)=c_{\left(\tilde{z}_{1}, \tilde{z}_{2}\right),\left(z_{1}, z_{2}\right)} \boldsymbol{A}_{W Z W}(\varphi, A)
$$

for $\varphi \in \operatorname{Map}_{z_{1}, z_{2}}\left(\mathcal{T}_{\tau}, G\right)$ which is a consequence of Eq. (4.9) (the minimally coupled term of the WZW action (4.24) is invariant under all gauge transformations).

As an example, let us consider the simplest gauged WZW model that exhibits a global gauge anomaly, namely the one with the target group $G=S U(3) / \mathbb{Z}_{3}$ at level $k=1$ and the gauged adjoint action of $\Gamma=G$. For the simple coweights of $s u(3)$ (identified with the simple weights), we may take

$$
\lambda_{1}^{\vee}=\operatorname{diag}\left[\frac{2}{3},-\frac{1}{3},-\frac{1}{3}\right]=\lambda_{1}, \quad \lambda_{2}^{\vee}=\operatorname{diag}\left[\frac{1}{3}, \frac{1}{3},-\frac{2}{3}\right]=\lambda_{2} .
$$

The element $z=\mathrm{e}^{2 \pi i \lambda_{1}^{\vee}}=\operatorname{diag}\left[\mathrm{e}^{\frac{4 \pi i}{3}}, \mathrm{e}^{-\frac{2 \pi i}{3}}, \mathrm{e}^{-\frac{2 \pi i}{3}}\right]$ generates the center $\mathbb{Z}_{3}$ of $S U(3)$. The set $P_{1}^{+}(s u(3))$ contains three weights $\Lambda=r_{1} \lambda_{1}+r_{2} \lambda_{2}$ with $\left(r_{1}, r_{2}\right)=(0,0),(1,0)$, $(0,1)$. We shall denote the corresponding level 1 affine characters by $\hat{\chi}_{\left(r_{1}, r_{2}\right)}$. The toroidal partition functions $\tilde{\mathcal{Z}}^{G}(\tau, u):=\mathcal{Z}^{G}(\tau, u) \exp \left[-\frac{\pi k \operatorname{tr}(u-\bar{u})^{2}}{2 \tau_{2}}\right]$ with fixed windings are, according to Eq. (4.35),

$$
\tilde{\mathcal{Z}}_{1,1}^{G}=\frac{1}{3}\left(\left|\hat{\chi}_{(0,0)}\right|^{2}+\left|\hat{\chi}_{(1,0)}\right|^{2}+\left|\hat{\chi}_{(0,1)}\right|^{2}\right)
$$




$$
\begin{aligned}
& \tilde{\mathcal{Z}}_{1, z}^{G}=\frac{1}{3}\left(\left|\hat{\chi}_{(0,0)}\right|^{2}+\mathrm{e}^{\frac{4 \pi i}{3}}\left|\hat{\chi}_{(1,0)}\right|^{2}+\mathrm{e}^{\frac{2 \pi i}{3}}\left|\hat{\chi}_{(0,1)}\right|^{2}\right), \\
& \tilde{\mathcal{Z}}_{1, z^{2}}^{G}=\frac{1}{3}\left(\left|\hat{\chi}_{(0,0)}\right|^{2}+\mathrm{e}^{\frac{2 \pi i}{3}}\left|\hat{\chi}_{(1,0)}\right|^{2}+\mathrm{e}^{\frac{4 \pi i}{3}}\left|\hat{\chi}_{(0,1)}\right|^{2}\right), \\
& \tilde{\mathcal{Z}}_{z, 1}^{G}=\frac{1}{3}\left(\hat{\chi}_{(0,1)} \overline{\hat{\chi}_{(0,0)}}+\hat{\chi}_{(0,0)} \overline{\hat{\chi}_{(1,0)}}+\hat{\chi}_{(1,0)} \overline{\hat{\chi}_{(0,1)}}\right), \\
& \tilde{\mathcal{Z}}_{z, z}^{G}=\frac{1}{3}\left(\mathrm{e}^{\frac{4 \pi i}{3}} \hat{\chi}_{(0,1)} \overline{\hat{\chi}_{(0,0)}}+\mathrm{e}^{\frac{2 \pi i}{3}} \hat{\chi}_{(0,0)} \overline{\hat{\chi}_{(1,0)}}+\hat{\chi}_{(1,0)} \overline{\hat{\chi}_{(0,1)}}\right), \\
& \tilde{\mathcal{Z}}_{z, z^{2}}^{G}=\frac{1}{3}\left(\mathrm{e}^{\frac{2 \pi i}{3}} \hat{\chi}_{(0,1)} \overline{\hat{\chi}_{(0,0)}}+\mathrm{e}^{\frac{4 \pi i}{3}} \hat{\chi}_{(0,0)} \overline{\hat{\chi}_{(1,0)}}+\hat{\chi}_{(1,0)} \overline{\hat{\chi}_{(0,1)}}\right), \\
& \tilde{\mathcal{Z}}_{z^{2}, 1}^{G}=\frac{1}{3}\left(\hat{\chi}_{(1,0)} \overline{\hat{\chi}_{(0,0)}}+\hat{\chi}_{(0,1)} \overline{\hat{\chi}_{(1,0)}}+\hat{\chi}_{(0,0)} \overline{\hat{\chi}_{(0,1)}}\right), \\
& \tilde{\mathcal{Z}}_{z^{2}, z}^{G}=\frac{1}{3}\left(\mathrm{e}^{\frac{2 \pi i}{3}} \hat{\chi}_{(1,0)} \overline{\hat{\chi}_{(0,0)}}+\hat{\chi}_{(0,1)} \overline{\hat{\chi}_{(1,0)}}+\mathrm{e}^{\frac{4 \pi i}{3}} \hat{\chi}_{(0,0)} \overline{\hat{\chi}_{(0,1)}}\right), \\
& \tilde{\mathcal{Z}}_{z^{2}, z^{2}}^{G}=\frac{1}{3}\left(\mathrm{e}^{\frac{4 \pi i}{3}} \hat{\chi}_{(1,0)} \overline{\hat{\chi}_{(0,0)}}+\hat{\chi}_{(0,1)} \overline{\hat{\chi}_{(1,0)}}+\mathrm{e}^{\frac{2 \pi i}{3}} \hat{\chi}_{(0,0)} \overline{\hat{\chi}_{(0,1)}}\right) .
\end{aligned}
$$

Since

$$
c_{\left(z^{\tilde{p}_{1}}, z^{\tilde{p}_{2}}\right),\left(z^{p_{1}}, z^{\left.p_{2}\right)}\right.}=\exp \left(\frac{4 \pi i}{3}\left(p_{1} \tilde{p}_{2}-\tilde{p}_{1} p_{2}\right)\right)
$$

the transformation rule (4.44) implies that all the sectors with non-trivial windings suffer from global gauge anomalies. Summing over the windings, one obtains the total partition function of the level $1 \mathrm{WZW}$ theory for the target group $G=S U(3) / \mathbb{Z}_{3}$ :

$$
\tilde{\mathcal{Z}}^{G}=\left|\hat{\chi}_{(0,0)}\right|^{2}+\hat{\chi}_{(1,0)} \overline{\hat{\chi}_{(0,1)}}+\hat{\chi}_{(0,1)} \overline{\hat{\chi}_{(1,0)}} \text {. }
$$

It should be contrasted with the anomaly-free level 1 partition function for the covering group $\tilde{G}=S U(3)$ :

$$
\tilde{\mathcal{Z}}^{\tilde{G}}=\left|\hat{\chi}_{(0,0)}\right|^{2}+\left|\hat{\chi}_{(1,0)}\right|^{2}+\left|\hat{\chi}_{(0,1)}\right|^{2}
$$

4.4. Implications for coset models. Consider now the group $\Gamma=\tilde{H} / Z_{\Gamma}$, where $\tilde{H}$ is a connected closed subgroup of $\tilde{G}$ with Lie algebra $\mathbf{h} \subset \mathbf{g}$ and $Z_{\Gamma}=\tilde{H} \cap \tilde{Z} . \Gamma=\tilde{\Gamma} / \tilde{Z}_{\Gamma}$, where, $\tilde{\Gamma}$ is the covering group of $\Gamma$ (and of $\tilde{H}$ ) and $\tilde{Z}_{\Gamma}$ is the subgroup of its center composed of elements that project to $Z_{\Gamma} \subset \tilde{H}$. Of course, one has to distinguish between $\tilde{Z}_{\Gamma}$ and $Z_{\Gamma}$ only if the subgroup $\tilde{H}$ is not simply connected. The so-called $G / \Gamma$ coset model of the conformal field theory is obtained by gauging the adjoint action of $\Gamma$ on $G=\tilde{G} / Z$ in the group $G$ level $k$ WZW model and by integrating out the gauge fields in the functional integral $[2,22,33,40]$. In particular, the contribution of the topologically trivial gauge fields to the toroidal partition function of the $G / \Gamma$ coset model is formally given by

$$
\mathcal{Z}^{G / \Gamma}(\tau)=\int \mathcal{Z}^{G}(\tau, A) D A
$$


where $A$ are 1 -forms on $\mathcal{T}_{\tau}$ with values in the Lie algebra $\mathbf{h}$. Clearly, due to the decomposition (4.31),

$$
\mathcal{Z}^{G / \Gamma}(\tau)=\sum_{\left(z_{1}, z_{2}\right) \in Z^{2}} \mathcal{Z}_{z_{1}, z_{2}}^{G / \Gamma}(\tau) \text { with } \mathcal{Z}_{z_{1}, z_{2}}^{G / \Gamma}(\tau)=\int \mathcal{Z}_{z_{1}, z_{2}}^{G}(\tau, A) D A
$$

The functional integral (4.50) may be computed by an appropriate parametrization of the gauge fields $A$ [22]. In particular, when $\mathbf{h}$ is semi-simple, the result is [22,33]

$$
\mathcal{Z}_{z_{1}, z_{2}}^{G / \Gamma}(\tau)=\frac{1}{\left|\tilde{Z}_{\Gamma}\right||Z|} \sum_{\Lambda \in P_{k}^{+}(\mathbf{g})} \sum_{\lambda \in P_{\tilde{k}}^{+}(\mathbf{h})} \epsilon_{z_{1}, z_{2}}(\Lambda) b_{k, z_{1}^{-1} \Lambda, \lambda}^{\hat{\mathbf{g}}, \hat{\mathbf{h}}}(\tau) \overline{b_{k, \Lambda, \lambda}^{\hat{\mathbf{g}}, \hat{\mathbf{h}}}(\tau)}
$$

where $b_{k, \Lambda, \lambda}^{\hat{\mathbf{g}}, \hat{\mathbf{h}}}(\tau)$ are the branching functions that are the characters of the coset Virasoro modules $V_{k, \Lambda, \lambda}^{\hat{\mathbf{g}}, \hat{\mathbf{h}}}$. The latter appear in the decomposition [29]

$$
V_{k, \Lambda}^{\hat{\mathbf{g}}}=\underset{\lambda \in P_{\tilde{k}}^{+}(\hat{\mathbf{h}})}{\bigoplus} V_{k, \Lambda, \lambda}^{\hat{\mathbf{g}, \hat{\mathbf{h}}}} \otimes V_{\tilde{k}, \lambda}^{\hat{\mathbf{h}}}
$$

of the level $k$ unitary highest-weight modules of the affine algebra $\hat{\mathbf{g}}$ into similar modules of the affine subalgebra $\hat{\mathbf{h}} \subset \hat{\mathbf{g}}$ at the level $\tilde{k}$ induced by restricting the bilinear form $k \operatorname{tr}$ on $\mathbf{g}$ to $\mathbf{h}$. By definition,

$$
b_{k, \Lambda, \lambda}^{\hat{\mathbf{g}}, \hat{\mathbf{h}}}(\tau)=\operatorname{tr}_{V_{k, \Lambda, \lambda}^{\hat{\mathbf{g}}, \hat{\mathbf{h}}}} \exp \left(2 \pi i \tau\left(L_{0}^{\hat{\mathbf{g}}}-L_{0}^{\hat{\mathbf{h}}}-\frac{c_{k}^{\hat{\mathbf{g}}}-c_{\tilde{k}}^{\hat{\mathbf{h}}}}{24}\right)\right) .
$$

The decomposition (4.53) implies the one for the characters:

$$
\chi_{k, \Lambda}^{\hat{\mathbf{g}}}(\tau, u)=\sum_{\lambda \in P_{\tilde{k}}^{+}(\mathbf{h})} b_{k, \Lambda, \lambda}^{\hat{\mathbf{g}, \hat{\mathbf{h}}}}(\tau) \chi_{\tilde{k}, \lambda}^{\hat{\mathbf{h}}}(\tau, u)
$$

for $u$ in the complexified Cartan algebra $\mathbf{t}_{\mathbf{h}}^{\mathbf{C}}$ of $\mathbf{h}$.

From the gauge transformation rule (4.44), we should expect that the sectors with fixed windings $\left(z_{1}, z_{2}\right)$ of the group $G$ WZW theory which transform in the anomalous

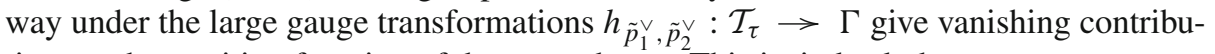
tions to the partition function of the coset theory. This is, indeed, the case.

Proposition 4.11. If $c_{\left(\tilde{z}_{1}, \tilde{z}_{2}\right),\left(z_{1}, z_{2}\right)} \neq 1$ for some $\left(\tilde{z}_{1}, \tilde{z}_{2}\right) \in Z_{\Gamma}^{2}$ then the partition function $\mathcal{Z}_{z_{1}, z_{2}}^{G / \Gamma}$ given by Eq. (4.52) vanishes.

Proof. Denote by $P_{\Gamma}^{\vee}$ the subset of the set $P_{\mathbf{h}}^{\vee} \subset i \mathbf{t}_{\mathbf{h}} \subset i \mathbf{t}_{\mathbf{g}}$ of coweights of $\mathbf{h}$ composed of such $\tilde{p}^{\vee}$ that $\tilde{z}=\mathrm{e}^{2 \pi i \tilde{p}^{\vee}} \in \tilde{Z}_{\Gamma}$ when viewed as elements of $\tilde{\Gamma}$ (or that $\tilde{z} \in Z_{\Gamma}$ when viewed as elements of $\tilde{H} \subset \tilde{G})$. Clearly $P_{\Gamma}^{\vee} \subset P_{\mathbf{g}}^{\vee}$. The vanishing result is a consequence of the following well known properties of the branching functions [16]:

$$
b_{k, \Lambda, \lambda}^{\hat{\mathbf{g}}, \hat{\mathbf{h}}}=0 \quad \text { if } \quad \exp \left[2 \pi i \operatorname{tr} \tilde{p}^{\vee} \Lambda\right] \neq \exp \left[2 \pi i \operatorname{tr} \tilde{p}^{\vee} \lambda\right] \quad \text { for some } \quad \tilde{p}^{\vee} \in P_{\Gamma}^{\vee},
$$




$$
b_{k, \tilde{z} \Lambda, \tilde{z} \lambda}^{\hat{\mathbf{g}}, \hat{\mathbf{h}}}=b_{k, \Lambda, \lambda}^{\hat{\mathbf{g}}, \hat{\mathbf{h}}} \quad \text { for } \quad \tilde{z}=\mathrm{e}^{2 \pi i \tilde{p}^{\vee}} \quad \text { and } \quad \tilde{p}^{\vee} \in P_{\Gamma}^{\vee}
$$

The first of these relations follows from the fact that the central elements $\mathrm{e}^{2 \pi i \tilde{p}^{\vee}}$ act by multiplication by the same scalars in the modules $V_{k, \Lambda}^{\hat{\mathbf{g}}}$ and $V_{\tilde{k}, \lambda}^{\hat{\mathbf{h}}}$ appearing in the decomposition (4.53). The second one is a consequence of the isomorphism between the coset Virasoro modules $V_{k, \Lambda, \lambda}^{\hat{\mathbf{g}}, \hat{\mathbf{h}}}$ with the weights related by the spectral flows under elements $\mathrm{e}^{2 \pi i \tilde{p}^{\vee}}$. Note that both relations are consistent with the fact that the identity (4.34) is satisfied by the characters of both affine algebras $\hat{\mathbf{g}}$ and $\hat{\mathbf{h}}$.

If $c_{\left(\tilde{z}_{1}, \tilde{z}_{2}\right),\left(z_{1}, z_{2}\right)} \neq 1$, then either $\exp \left[2 \pi i k \operatorname{tr} p_{1}^{\vee} \tilde{p}_{2}^{\vee}\right] \neq 1$ or $\exp \left[-2 \pi i k \operatorname{tr} \tilde{p}_{1}^{\vee} p_{2}^{\vee}\right] \neq 1$ for some $\tilde{p}_{i}^{\vee} \in P_{\Gamma}^{\vee}$, see Eq. (4.22). Relation (4.56) implies that if $\exp \left[2 \pi i k \operatorname{tr} p_{1}^{\vee} \tilde{p}_{2}^{\vee}\right]=$ $\exp \left[-2 \pi i \operatorname{tr} \tilde{p}_{2}^{\vee}\left(z_{1}^{-1} \Lambda-\Lambda\right)\right] \neq 1$ for some $\tilde{p}_{2}^{\vee} \in P_{\Gamma}^{\vee}$, then, for each pair $(\Lambda, \lambda)$, either $b_{k, z_{1}^{-1} \Lambda, \lambda}^{\hat{\mathbf{g}}, \hat{\mathbf{h}}}=0$ or $b_{k, \Lambda, \lambda}^{\hat{\mathbf{g}}, \hat{\mathbf{h}}}=0$ so that $\mathcal{Z}_{z_{1}, z_{2}}^{G / \Gamma}$ vanishes. Similarly, using relation (4.57) and Eq. (4.43), we infer that if $\exp \left[-2 \pi i k \operatorname{tr} \tilde{p}_{1}^{\vee} p_{2}^{\vee}\right] \neq 1$ for some $\tilde{p}_{1}^{\vee} \in P_{\Gamma}^{\vee}$, then $\mathcal{Z}_{z_{1}, z_{2}}^{G / \Gamma}$ vanishes too.

As we see, global gauge anomalies in the WZW model lead to selection rules for the contributions to the partition functions of the $G / \Gamma$ coset model.

Let $Z^{\prime} \subset Z$ be the non-anomalous subgroup that is composed of the elements $z=$ $\mathrm{e}^{2 \pi i p^{\vee}} \in Z$ such that $\exp \left[2 \pi i k \operatorname{tr} p^{\vee} \tilde{p}^{\vee}\right]=1$ for all $\tilde{p}^{\vee} \in P_{\Gamma}^{\vee}$, and let $G^{\prime}=\tilde{G} / Z^{\prime}$ be the corresponding quotient of $\tilde{G}$. Proposition 4.11 and Eqs. (4.52) imply that

$$
\mathcal{Z}^{G / \Gamma}(\tau)=\frac{\left|Z^{\prime}\right|}{|Z|} \mathcal{Z}^{G^{\prime} / \Gamma}(\tau)
$$

Upon summation over windings in $\left(Z^{\prime}\right)^{2}$, the partition function on the right-hand side may be rewritten in the form

$$
\mathcal{Z}^{G^{\prime} / \Gamma}(\tau)=\frac{1}{\left|\tilde{Z}_{\Gamma}\right|} \sum_{z \in Z^{\prime}} \sum_{\Lambda \in P_{k}^{+}(\mathbf{g}) \cap C_{z}^{\prime}} \sum_{\lambda \in P_{\tilde{k}}^{+}(\mathbf{h})} b_{k, z^{-1} \Lambda, \lambda}^{\hat{\mathbf{g}, \hat{\mathbf{h}}}}(\tau) \overline{b_{k, \Lambda, \lambda}^{\hat{\mathbf{g}}, \hat{\mathbf{h}}}(\tau)},
$$

where $C_{z}^{\prime}$ is defined as in (4.37) but with the subgroup $Z$ replaced by $Z^{\prime}$. Due to relation (4.56), we may restrict the sum on the right-hand side to pairs $(\Lambda, \lambda)$ such that the elements of $\mathrm{e}^{2 \pi i \tilde{p}^{\vee}}$ for $\tilde{p}^{\vee} \in P_{\Gamma}^{\vee}$ act by multiplication by the same scalar in $V_{k, \Lambda}^{\hat{\mathbf{g}}}$ and in $V_{\tilde{k}, \lambda}^{\hat{\mathbf{h}}}$. Then, also the pairs $\left(z^{-1} \Lambda, \lambda\right)$ for $z \in Z^{\prime}$ and $(\tilde{z} \Lambda, \tilde{z} \lambda)$ for $\tilde{z}=\mathrm{e}^{2 \pi i \tilde{p}^{\vee}}$ will have this property due to Eq. (4.42). Besides, it follows from Eq. (4.43) that if $\Lambda \in C_{z}^{\prime}$ then $\tilde{z} \Lambda \in C_{z}^{\prime}$ for all $\tilde{z} \in Z_{\Gamma}$ (unlike for $C_{z}$ if $Z^{\prime}$ is strictly smaller than $Z$ ). As a result of this observation and of relation (4.57), one may rewrite the sum over weights on the right-hand side of Eq. (4.59) as a sum over orbits $[\Lambda, \lambda]$ of the diagonal spectral flow of $\tilde{Z}_{\Gamma}$. Denoting by $\mathcal{P}_{z}$ the space of such orbits with $\Lambda \in C_{z}^{\prime}$, we infer that

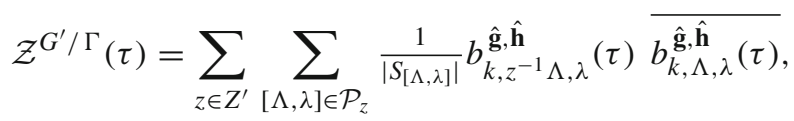

where $S_{[\Lambda, \lambda]} \subset \tilde{Z}_{\Gamma}$ denotes the stabilizer subgroup of the elements of the orbit $[\Lambda, \lambda]$. If $S_{[\Lambda, \lambda]}$ is trivial for all orbits $[\Lambda, \lambda]$ then the last expression for the partition function 
$\mathcal{Z}^{G^{\prime} / \Gamma}(\tau)$ is consistent with the following form of the space of states:

$$
\mathbb{H}^{G^{\prime} / \Gamma}=\bigoplus_{z \in Z^{\prime}}\left(\underset{[\Lambda, \lambda] \in \mathcal{P}_{z}}{\bigoplus} V_{k, z^{-1} \Lambda, \lambda}^{\hat{\mathbf{g}}, \hat{\mathbf{h}}} \otimes \overline{V_{k, \Lambda, \lambda}^{\hat{\mathbf{g}}, \hat{\mathbf{h}}}}\right)
$$

Identity (4.58) now implies that, on the contrary, barring further identifications of the coset Virasoro representations [9], the partition function $Z^{G / \Gamma}(\tau)$ lacks a Hilbert-space interpretation if the group $Z^{\prime}$ is strictly smaller than $Z$, i.e. if the group $G$ WZW model suffers from global gauge anomalies relative to the adjoint action of $\Gamma$. This points to the inconsistency of the $G / \Gamma$ coset model in that case. On the level of the partition function, this inconsistency is of a mild nature since one may turn the inconsistent partition function $\mathcal{Z}^{G / \Gamma}$ into the consistent one $\mathcal{Z}^{G^{\prime} / \Gamma}$ by changing the normalization.

In the case when $G=S U(3) / \mathbb{Z}_{3}=\Gamma$, the $G / \Gamma$ coset theory is topological and its partition function is $\tau$-independent. The branching functions vanish if $\Lambda \neq \lambda$ and are equal to 1 otherwise. At level 1 , all coset partition functions with non-trivial windings vanish and

$$
\mathcal{Z}^{G / \Gamma}=\mathcal{Z}_{1,1}^{G / \Gamma}=\frac{1}{3} .
$$

In a consistent two-dimensional topological field theory, the partition function is equal to the dimension of the space of states and cannot take a fractional value, confirming the inconsistency of the level $1 G / \Gamma$ coset model for $G=S U(3) / \mathbb{Z}_{3}=\Gamma$. On the other hand, the non-anomalous subgroup $Z^{\prime} \subset Z=\mathbb{Z}_{3}$ is trivial so that $G^{\prime}=\tilde{G}=S U(3)$ in that case, and for the anomaly-free level $1 \tilde{G} / \Gamma$ coset theory,

$$
\mathcal{Z}^{\tilde{G} / \Gamma}=1
$$

corresponding to a 1-dimensional space of states.

It was pointed out in [47] (for the diagonal coset models corresponding to simply connected groups $\left.G=\tilde{G}=G^{\prime}\right)$ that, in the presence of fixed points $\left(\Lambda_{0}, \lambda_{0}\right)$ of the diagonal spectral flow of $\tilde{Z}_{\Gamma}$, there is a further problem with the Hilbert space interpretation of the partition function (4.60) because of the appearance of the fraction $\frac{1}{{ }_{\left[\Lambda_{0}, \lambda_{0}\right]}}$. It was shown in [16] within an algebraic approach how to resolve such fixed points to repair this defect. Somewhat earlier, in [33], it was argued that the problem may be resolved on the Lagrangian level by adding to the partition function (4.60) contributions from the sectors with gauge fields in the topologically non-trivial principal $\Gamma$-bundles $P$ over $\mathcal{T}_{\tau}$ (it was also shown that such contributions vanish if there are no fixed points $\left(\Lambda_{0}, \lambda_{0}\right)$ of the diagonal spectral flow of $\left.\tilde{Z}_{\Gamma}\right)$. For the sectors with topologically non-trivial gauge fields, the WZW sigma model fields are sections of the associated bundle $P \times_{\Gamma} G$ with respect to the adjoint action of $\Gamma$, and the globally gauge invariant WZW amplitudes in the gauge field background may be defined with the help of a $\Gamma$-equivariant structure on the WZW gerbe $\mathcal{G}_{k}$ over $G$, as will be explained in the following section.

\section{Coupling to General Gauge Fields}

5.1. Equivariant gerbes. We showed in Sect. 3 that the invariance of the Feynman amplitudes (3.10) under all gauge transformations requires the existence of a 1-isomorphism between the gerbes $\mathcal{G}_{12} \equiv \ell^{*} \mathcal{G}$ and $\mathcal{I}_{\rho} \otimes \mathcal{G}_{2}$ over $\Gamma \times M$. Here, we shall strengthen this property by introducing the notion of $\Gamma$-equivariant gerbes in the way that will subsequently assure the gauge invariance of the Feynman amplitudes coupled to topologically non-trivial gauge fields. 
Definition 5.1. A gerbe $\mathcal{G}$ with the curvature $H$ possessing a $\Gamma$-equivariantly closed extension $\hat{H}(X)=H+v(X)$ will be called $\Gamma$-equivariant relative to the 2-form $\rho$ given by Eq. (3.26) if it is equipped with a pair $(\alpha, \beta)$, called a $\Gamma$-equivariant structure, such that

(i) $\alpha: \mathcal{G}_{12} \rightarrow \mathcal{I}_{\rho} \otimes \mathcal{G}_{2}$ is a 1-isomorphism of gerbes over $\Gamma \times M$;

(ii) $\beta:\left(I d \otimes \alpha_{2,3}\right) \circ \alpha_{1,23} \Rightarrow \alpha_{12,3}$ is a 2-isomorphism of 1-isomorphisms of gerbes over $\Gamma^{2} \times M$

(iii) the following diagram of 2-isomorphisms between 1-isomorphisms of gerbes over $\Gamma^{3} \times M$ is commutative:

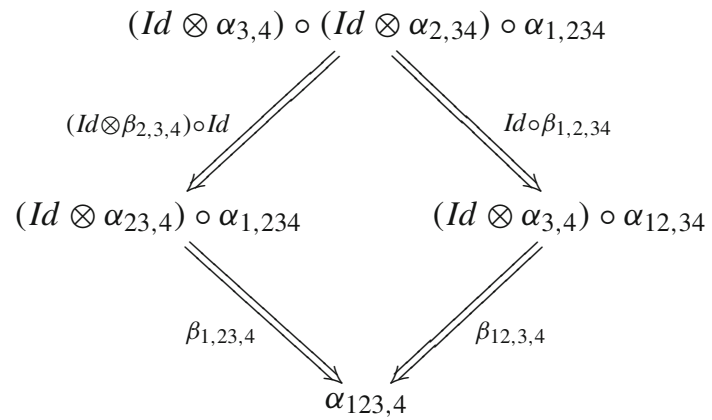

$\Gamma$-equivariant gerbes over $M$ form a 2-category $\operatorname{Gr} b^{\nabla}(M)^{G}$. A 1-isomorphism between two $\Gamma$-equivariant gerbes,

$$
(\chi, \eta):\left(\mathcal{G}^{a}, \alpha^{a}, \beta^{a}\right) \rightarrow\left(\mathcal{G}^{b}, \alpha^{b}, \beta^{b}\right),
$$

is a 1-isomorphism $\chi: \mathcal{G}^{a} \rightarrow \mathcal{G}^{b}$ and a 2-isomorphism $\eta:\left(\operatorname{Id} \otimes \chi_{2}\right) \circ \alpha^{a} \Rightarrow \alpha^{b} \circ \chi_{12}$ between 1 -isomorphisms of gerbes over $\Gamma \times M$, such that the diagram

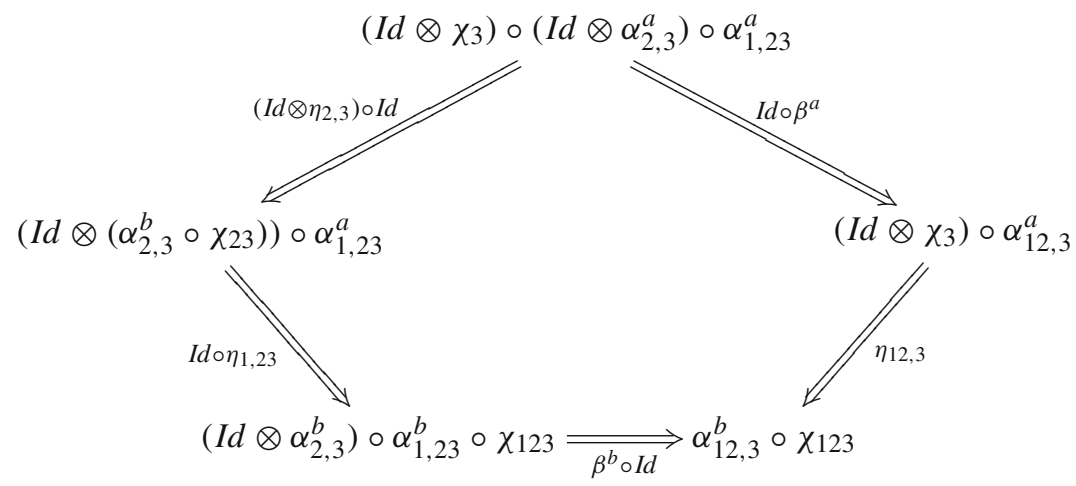

of 2-isomorphisms between 1-isomorphisms of gerbes over $\Gamma^{2} \times M$ is commutative. 1 -isomorphic $\Gamma$-equivariant gerbes necessarily correspond to the same curvature $H$ and to the same 2-form $\rho$ and, consequently, to the same $\Gamma$-equivariantly closed extension $\hat{H}$. The identity 1 -isomorphism of $\Gamma$-equivariant gerbes is given by the pair $(\chi, \eta)=$ $(I d, I d)$ for which the diagram (5.3) reduces to a trivially commutative one. Finally, a $\Gamma$-equivariant 2-isomorphism

$$
\epsilon:(\chi, \eta) \Rightarrow\left(\chi^{\prime}, \eta^{\prime}\right)
$$


is a 2-isomorphism $\epsilon: \chi \Rightarrow \chi^{\prime}$ such that the diagram

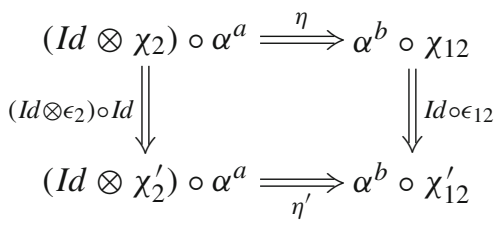

is commutative, which is trivially the case for the identity 2-isomorphism $I d: \chi \Rightarrow \chi$ when also $\eta^{\prime}=\eta$.

Remark 5.2. 1. We shall say that two $\Gamma$-equivariant structures $\left(\alpha^{a}, \beta^{a}\right)$ and $\left(\alpha^{b}, \beta^{b}\right)$ on the gerbe $\mathcal{G}$ are isomorphic if the $\Gamma$-equivariant gerbes $\left(\mathcal{G}, \alpha^{a}, \beta^{a}\right)$ and $\left(\mathcal{G}, \alpha^{b}\right.$, $\left.\beta^{b}\right)$ are 1-isomorphic.

2. If $\left(\mathcal{G}^{a}, \alpha^{a}, \beta^{a}\right)$ is a $\Gamma$-equivariant gerbe, then for each 1 -isomorphism of gerbes $\delta: \mathcal{G}^{a} \rightarrow \mathcal{G}^{b}$ there exists a $\Gamma$-equivariant structure $\left(\alpha^{b}, \beta^{b}\right)$ on $\mathcal{G}^{b}$ such that the $\Gamma$-equivariant gerbes $\left(\mathcal{G}^{a}, \alpha^{a}, \beta^{a}\right)$ and $\left(\mathcal{G}^{b}, \alpha^{b}, \beta^{b}\right)$ are 1 -isomorphic.

3. $\Gamma$-equivariant gerbes $(\mathcal{G}, \alpha, \beta)$ over a $\Gamma$-space $M$ may be pulled back to $\Gamma$-equivariant gerbes $\left(f^{*} \mathcal{G}, f_{2}^{*} \alpha, f_{3}^{*} \beta\right)$ over another $\Gamma$-space $N$ along $\Gamma$-equivariant maps $f: N \rightarrow M$. Similarly, their 1- and 2-isomorphisms may be pulled back.

4. For any subgroup $\Gamma^{\prime} \subset \Gamma$, the restriction induces a $\Gamma^{\prime}$-equivariant gerbe from a $\Gamma$-equivariant gerbe $(\mathcal{G}, \alpha, \beta)$.

5. The concept of equivariant (bundle) gerbes (with connection) introduced here is different, although not unrelated, to the one discussed in [42]. For discrete groups $\Gamma$, the above definitions of $\Gamma$-equivariant gerbes and their 1-isomorphisms and 2-isomorphisms are equivalent to those introduced in [26] (where the actions of $\Gamma$ that change the sign of the curvature 3-form $H$ were also considered).

There is a sub-2-category $\operatorname{Grb}^{\nabla}(M)_{0}^{G}$ composed of those $\Gamma$-equivariant gerbes $\mathcal{G}$ whose curvature $H$ is $\Gamma$-equivariantly closed and the 2 -form $\rho=0$. Below, we shall need the following result, a particular consequence of the general descent theory for gerbes:

Theorem 5.3. Suppose that $\Gamma$ acts on $M$ in such a way that $M^{\prime}=M / \Gamma$ is a smooth manifold and $M$ forms a smooth (left) principal $\Gamma$-bundle $\omega: M \rightarrow M^{\prime}$. Then, there exists a canonical equivalence

$$
G r b^{\nabla}(M)_{0}^{\Gamma} \cong G r b^{\nabla}\left(M^{\prime}\right) .
$$

In particular, a gerbe $\mathcal{G}$ over $M$ that is $\Gamma$-equivariant relative to the zero 2 -form descends to a gerbe $\mathcal{G}^{\prime}$ over $M^{\prime}$ whose pullback by $\omega$ is 1 -isomorphic to $\mathcal{G}$. The equivalence of Theorem 5.3 commutes with the pullback functors: $f^{*}$ of $G r b^{\nabla}(M)_{0}^{\Gamma}$ induced by a $\Gamma$-equivariant map $f: N \rightarrow M$ and $f^{\prime *}$ of $G r b^{\nabla}\left(M^{\prime}\right)$ induced by the projected $\operatorname{map} f^{\prime}: N^{\prime} \rightarrow M^{\prime}$.

We give a proof of Theorem 5.3 in Appendix 4, employing results of [50].

5.2. WZ amplitudes with topologically non-trivial gauge fields. In Sect. 3 , we discussed only topologically trivial two-dimensional gauge fields, i.e. connections in the trivial 
principal $\Gamma$-bundle over the worldsheet $\Sigma$. Here, we shall consider connections in a general principal $\Gamma$-bundle $\pi: P \rightarrow \Sigma$. Such connections correspond to g-valued 1-forms $\mathcal{A}$ on $P$ with the following defining property:

$$
\left(r^{*} \mathcal{A}\right)\left(p, \gamma^{-1}\right)=A d_{\gamma}(\mathcal{A}(p)-\Theta(\gamma)),
$$

where $r: P \times \Gamma \rightarrow P$ is the right action of $\Gamma$ on $P$. For a $\Gamma$-equivariantly closed 3-form $\hat{H}(X)=H+v(X)$, consider the 2 -form $\tilde{\rho}_{\mathcal{A}}$ on $\tilde{M}:=P \times M$ given by the formula

$$
\tilde{\rho}_{\mathcal{A}}:=-v(\mathcal{A})+\frac{1}{2} \iota \overline{\mathcal{A}} v(\mathcal{A})
$$

compare to the first of Eqs. (3.9). Below, the map $\tilde{\ell}: \Gamma \times \tilde{M} \rightarrow \tilde{M}$ will denote the left action of $\Gamma$ on $\tilde{M}$ :

$$
\tilde{\ell}(\gamma,(p, m)):=\left(r\left(p, \gamma^{-1}\right), \ell(\gamma, m)\right)=\left(p \gamma^{-1}, \gamma m\right) .
$$

For maps and forms on the product spaces $\Gamma^{n} \times \tilde{M}$, we shall use the notation from the beginning of Sect. 3.3, marking the subscript indices with a tilde. The subscript indices without a tilde will be reserved for the factors in the expanded expression $\Gamma^{n} \times P \times M$ for the same spaces. One has the following counterpart of Eq. (3.31):

Lemma 5.4. As forms on $\Gamma \times \tilde{M}=\Gamma \times P \times M$,

$$
\left(\tilde{\rho}_{\mathcal{A}}\right)_{\tilde{1} \tilde{2}}=\left(\tilde{\rho}_{\mathcal{A}}\right)_{2,3}-\rho_{1,3}=\left(\tilde{\rho}_{\mathcal{A}}\right)_{\tilde{2}}-\rho_{1,3}
$$

A proof of Lemma 5.4 is given in Appendix 5.

Let $\mathcal{G}$ be a gerbe over $M$ with the curvature $H$ which extends to the $\Gamma$-equivariantly closed form $\hat{H}=H+v(X)$. Define a gerbe $\tilde{\mathcal{G}}_{\mathcal{A}}$ over $\tilde{M}=P \times M$ by setting

$$
\tilde{\mathcal{G}}_{\mathcal{A}}:=\mathcal{I}_{\tilde{\rho}_{\mathcal{A}}} \otimes \mathcal{G}_{2}
$$

Note that the curvature of $\tilde{\mathcal{G}}_{\mathcal{A}}$ is given by the closed 3 -form

$$
\tilde{H}_{\mathcal{A}}:=d \tilde{\rho}_{\mathcal{A}}+H_{2}
$$

For the pullback of $\tilde{H}_{\mathcal{A}}$ under the action $\tilde{\ell}$ of $\Gamma$ on $\tilde{M}$, we obtain from Lemmas 5.4 and 3.11:

$$
\left(\tilde{H}_{\mathcal{A}}\right)_{1 \tilde{2}}=d\left(\tilde{\rho}_{\mathcal{A}}\right)_{\tilde{1} \tilde{2}}+\left(\ell^{*} H\right)_{1,3}=d\left(\tilde{\rho}_{\mathcal{A}}\right)_{\tilde{2}}-d \rho_{1,3}+d \rho_{1,3}+H_{3}=\left(\tilde{H}_{\mathcal{A}}\right)_{2} .
$$

It follows that $\tilde{H}_{\mathcal{A}}$ (without any further extension) is a $\Gamma$-equivariantly closed form on $\tilde{M}$.

Proposition 5.5. Let $(\mathcal{G}, \alpha, \beta)$ be a $\Gamma$-equivariant gerbe over $M$ in the sense of Definition 5.1 and let $P$ be a principal $\Gamma$-bundle over the surface $\Sigma$ with connection $\mathcal{A}$. Then the gerbe $\tilde{\mathcal{G}}_{\mathcal{A}}$ over $\tilde{M}=P \times M$ may be canonically equipped with the structure of a $\Gamma$-equivariant gerbe relative to the zero 2-form. 
Proof. First, we have to construct a 1-isomorphism $\tilde{\alpha}_{\mathcal{A}}$ of gerbes over $\Gamma \times \tilde{M}$ :

$$
\tilde{\alpha}_{\mathcal{A}}:\left(\tilde{\mathcal{G}}_{\mathcal{A}}\right)_{\tilde{1} \tilde{2}} \rightarrow\left(\tilde{\mathcal{G}}_{\mathcal{A}}\right)_{\tilde{2}}
$$

It is obtained as the composition

$$
\left(\tilde{\mathcal{G}}_{\mathcal{A}}\right)_{\tilde{1} \tilde{2}}=\mathcal{I}_{\left(\tilde{\rho}_{\mathcal{A}}\right)_{\tilde{1} \tilde{2}}} \otimes \mathcal{G}_{13} \stackrel{I d \otimes \alpha_{1,3}}{\longrightarrow} \mathcal{I}_{\left(\tilde{\rho}_{\mathcal{A}}\right)_{\tilde{1} \tilde{2}}} \otimes \mathcal{I}_{\rho_{1,3}} \otimes \mathcal{G}_{3}=\mathcal{I}_{\left(\tilde{\rho}_{\mathcal{A}}\right)_{2}} \otimes \mathcal{G}_{3}=\left(\tilde{\mathcal{G}}_{\mathcal{A}}\right)_{\tilde{2}},
$$

where we used Lemma 5.4. Hence, $\tilde{\alpha}_{\mathcal{A}}$ is the tensor product of the identity 1 -isomorphism of the gerbe $\mathcal{I}_{\left(\tilde{\rho}_{\mathcal{A}}\right)_{\tilde{1} 2}}$ with the 1-isomorphism $\alpha_{1,3}$.

Next, we have to construct a 2 -isomorphism $\tilde{\beta}_{\mathcal{A}}$ between 1 -isomorphisms of gerbes $\left(\tilde{\mathcal{G}}_{\mathcal{A}}\right)_{\tilde{1} \tilde{2} \tilde{3}}$ and $\left(\tilde{\mathcal{G}}_{\mathcal{A}}\right)_{\tilde{3}}$ over $\Gamma^{2} \times \tilde{M}$,

$$
\tilde{\beta}_{\mathcal{A}}:\left(\tilde{\alpha}_{\mathcal{A}}\right)_{\tilde{2}, \tilde{3}} \circ\left(\tilde{\alpha}_{\mathcal{A}}\right)_{\tilde{1}, \tilde{2} \tilde{3}} \Rightarrow\left(\tilde{\alpha}_{\mathcal{A}}\right)_{\tilde{1} \tilde{2}, \tilde{3}} .
$$

Note that $\left(\tilde{\alpha}_{\mathcal{A}}\right)_{\tilde{1}, \tilde{2} \tilde{3}}$ is the 1-isomorphism

$$
\begin{aligned}
\left(\tilde{\mathcal{G}}_{\mathcal{A}}\right)_{\tilde{1} \tilde{2} \tilde{3}} & =\mathcal{I}_{\left(\tilde{\rho}_{\mathcal{A}}\right)_{\tilde{1} \tilde{3} \tilde{3}}} \otimes \mathcal{G}_{124} \stackrel{I d \otimes \alpha_{1,24}}{\longrightarrow} \mathcal{I}_{\left(\tilde{\rho}_{\mathcal{A}}\right)_{\tilde{1} \tilde{2} \tilde{3}}} \otimes \mathcal{I}_{\rho_{1,24}} \otimes \mathcal{G}_{24} \\
& =\mathcal{I}_{\left(\tilde{\rho}_{\mathcal{A}}\right)_{\tilde{2} \tilde{3}}} \otimes \mathcal{G}_{24}=\left(\tilde{\mathcal{G}}_{\mathcal{A}}\right)_{\tilde{2} \tilde{3}},
\end{aligned}
$$

since Lemma 5.4 implies that $\left(\tilde{\rho}_{\mathcal{A}}\right)_{\tilde{1} \tilde{2} \tilde{3}}+\rho_{1,24}=\left(\tilde{\rho}_{\mathcal{A}}\right)_{\tilde{2} \tilde{3}}$. Similarly, $\left(\tilde{\alpha}_{\mathcal{A}}\right)_{\tilde{2}, \tilde{3}}$ is the 1-isomorphism

$$
\begin{aligned}
\left(\tilde{\mathcal{G}}_{\mathcal{A}}\right)_{\tilde{2} \tilde{3}} & =\mathcal{I}_{\left(\tilde{\rho}_{\mathcal{A}}\right)_{\tilde{2} \tilde{3}}} \otimes \mathcal{G}_{24} \stackrel{I d \otimes \alpha_{2,4}}{\longrightarrow} \mathcal{I}_{\left(\tilde{\rho}_{\mathcal{A}}\right)_{\tilde{2} \tilde{3}}} \otimes \mathcal{I}_{\rho_{2,4}} \otimes \mathcal{G}_{4} \\
& =C I_{\left(\tilde{\rho}_{\mathcal{A}}\right)_{\tilde{3}}} \otimes \mathcal{G}_{4}=\left(\tilde{\mathcal{G}}_{\mathcal{A}}\right)_{\tilde{3}},
\end{aligned}
$$

where we used the relation $\left(\tilde{\rho}_{\mathcal{A}}\right)_{\tilde{2} \tilde{3}}+\rho_{2,4}=\left(\tilde{\rho}_{\mathcal{A}}\right)_{\tilde{3}}$, again following from Lemma 5.4. Hence, $\left(\tilde{\alpha}_{A}\right)_{2}, \tilde{3}$ ○ $\left(\tilde{\alpha}_{\mathcal{A}}\right)_{\tilde{1}, \tilde{2} \tilde{3}}$ is the 1-isomorphism

$$
\begin{aligned}
& \mathcal{I}_{\left(\tilde{\rho}_{\mathcal{A}}\right)_{\tilde{1} \tilde{2} \tilde{3}}} \otimes \mathcal{G}_{124} \stackrel{I d \otimes \alpha_{1,24}}{\longrightarrow} \mathcal{I}_{\left(\tilde{\rho}_{\mathcal{A}}\right)_{\tilde{1} \tilde{2} \tilde{3}}} \otimes \mathcal{I}_{\rho_{1,24}} \otimes \mathcal{G}_{24} \\
& \quad \stackrel{I d \otimes \alpha_{2,4}}{\longrightarrow} \mathcal{I}_{\left(\tilde{\rho}_{\mathcal{A}}\right)_{\tilde{1} \tilde{2} \tilde{3}}} \otimes \mathcal{I}_{\rho_{1,24}} \otimes \mathcal{I}_{\rho_{2,4}} \otimes \mathcal{G}_{4}=\mathcal{I}_{\left(\tilde{\rho}_{\mathcal{A}}\right)_{3}} \otimes \mathcal{G}_{4},
\end{aligned}
$$

that is the tensor product of the identity 1-isomorphism of the gerbe $\mathcal{I}_{\left(\tilde{\rho}_{\mathcal{A}}\right)_{\tilde{1} \tilde{2} \tilde{3}}}$ with the 1-isomorphism $\left(I d \otimes \alpha_{2,4}\right) \circ \alpha_{1,24}$. On the other hand, $\left(\tilde{\alpha}_{\mathcal{A}}\right)_{\tilde{1} \tilde{2}, \tilde{3}}$ is the 1 -isomorphism given by

$$
\begin{aligned}
\left(\tilde{\mathcal{G}}_{\mathcal{A}}\right)_{\tilde{1} \tilde{2} \tilde{3}} & =\mathcal{I}_{\left(\tilde{\rho}_{\mathcal{A}}\right)_{\tilde{1} \tilde{2} \tilde{3}}} \otimes \mathcal{G}_{124} \stackrel{I d \otimes \alpha_{12,4}}{\longrightarrow} \mathcal{I}_{\left(\tilde{\rho}_{\mathcal{A}}\right)_{\tilde{1} \tilde{2} \tilde{3}}} \otimes \mathcal{I}_{\rho_{12,4}} \otimes \mathcal{G}_{4} \\
& =\mathcal{I}_{\left(\rho_{\mathcal{A}}\right)_{\tilde{3}}} \otimes \mathcal{G}_{4}=\left(\tilde{\mathcal{G}}_{\mathcal{A}}\right)_{\tilde{3}}
\end{aligned}
$$

because $\left(\tilde{\rho}_{\mathcal{A}}\right)_{\tilde{1} 2} \tilde{3}+\rho_{12,4}=\left(\tilde{\rho}_{\mathcal{A}}\right)_{\tilde{3}}$, once again by virtue of Lemma 5.4. Comparison between (5.19) and (5.20), and Definition 5.1 (ii) show that we may take for $\tilde{\beta}_{\mathcal{A}}$ the 2 -isomorphism obtained by tensoring the identity 2 -isomorphism between the identity 1-isomorphisms of the gerbe $\mathcal{I}_{\left(\tilde{\rho}_{\mathcal{A}}\right)_{\tilde{1} \tilde{2} \tilde{3}}}$ with the 2-isomorphism $\beta_{1,2,4}$ :

$$
\tilde{\beta}_{\mathcal{A}}:=I d \otimes \beta_{1,2,4} .
$$


We have to check that the 1-isomorphism $\tilde{\alpha}_{\mathcal{A}}$ and 2-isomorphism $\tilde{\beta}_{\mathcal{A}}$ make the diagram

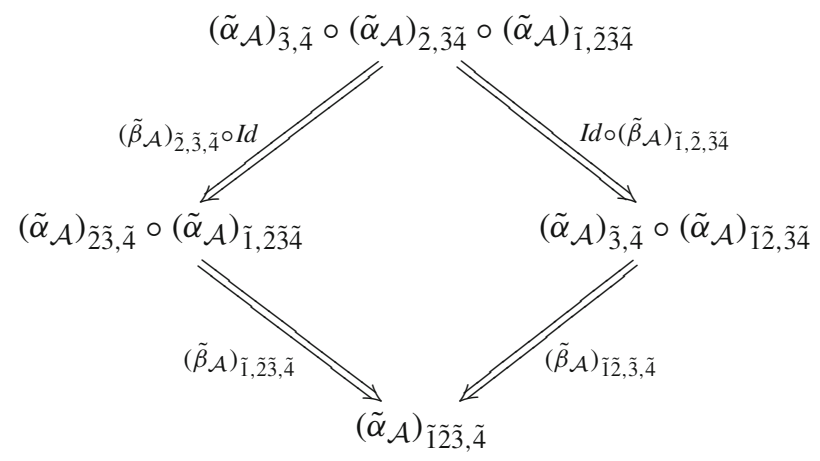

commutative. It is easy to see that the above diagram may be identified with the tensor product of the identity 2 -isomorphism between the identity 1 -isomorphisms of the gerbe $\mathcal{I}_{\left(\tilde{\rho}_{\mathcal{A}}\right)_{\tilde{1} \tilde{2} \tilde{3} \tilde{4}}}$ by the pullback of diagram (5.1) along the projection from $\Gamma^{3} \times P \times M$ to $\Gamma^{3} \times M$. This assures its commutativity, completing the proof of Proposition 5.5.

The action (5.9) of $\Gamma$ on $\tilde{M}$ is free and the quotient space $\tilde{M} / \Gamma=P \times_{\Gamma} M=: P_{M}$ is the associated bundle over $\Sigma$ with the typical fiber $M$. The space $\tilde{M}$ may be viewed as a (left) principal $\Gamma$-bundle $\tilde{\omega}: \tilde{M} \rightarrow P_{M}$. Theorem 5.3 and Proposition 5.5 have as the immediate consequence

Corollary 5.6. The gerbe $\tilde{\mathcal{G}}_{\mathcal{A}}$ on $\tilde{M}$ descends to a gerbe $\mathcal{G}_{\mathcal{A}}$ on $P_{M}$ whose pullback along $\tilde{\omega}$ is 1-isomorphic to $\tilde{\mathcal{G}}_{\mathcal{A}}$. In particular, the curvature of $\mathcal{G}_{\mathcal{A}}$ is equal to the closed 3-form $H_{\mathcal{A}}$ on $P_{M}$ whose pullback to $\tilde{M}$ coincides with $\tilde{H}_{\mathcal{A}}$.

In order to couple the sigma model with target $M$ to a gauge field $\mathcal{A}$ in the principal $\Gamma$-bundle $\pi: P \rightarrow \Sigma$, one has to modify also the sigma-model fields. In the gauged model, they become global sections $\Phi: \Sigma \rightarrow P_{M}$ of the associated bundle rather than maps from $\Sigma$ to $M$.

Definition 5.7. Let $(\mathcal{G}, \alpha, \beta)$ be a $\Gamma$-equivariant gerbe over $M$ and $P$ a principal $\Gamma$-bundle with connection $\mathcal{A}$ over a closed oriented surface $\Sigma$. The Wess-Zumino contribution of a field $\Phi: \Sigma \rightarrow P_{M}$ to the gauged Feynman amplitude is defined by

$$
\boldsymbol{A}_{W Z}(\Phi, \mathcal{A}):=\operatorname{Hol}_{\mathcal{G}_{\mathcal{A}}}(\Phi)
$$

Remark 5.8. The above constructions are functorial with respect to isomorphisms of principal bundles $P$. If $P$ is trivial, i.e. $P=\Sigma \times \Gamma$, then the gauge fields $\mathcal{A}$ may be related to $g$-valued 1-forms $A$ on $M$ by the formula $\mathcal{A}\left(x, \gamma^{-1}\right)=A d_{\gamma}(A(x)-\Theta(\gamma))$. In this case, the associated bundle $P_{M}$ may be naturally identified with $\Sigma \times M$, and the gerbe $\mathcal{G}_{\mathcal{A}}$ with the gerbe $\mathcal{G}_{A}$ defined by relation (3.9). One recovers this way the coupling to the topologically trivial gauge fields discussed previously, see Definition 3.3.

5.3. General gauge invariance. For the general case of gauge fields $\mathcal{A}$ corresponding to connections in a principal $\Gamma$-bundle $\pi: P \rightarrow \Sigma$, the general gauge transformations $h$ are defined as sections of the associated bundle $A d(P)=P \times_{A d} \Gamma$. The latter is 
composed of the orbits $\left\{\left(p \gamma^{\prime-1}, A d_{\gamma}^{\prime}(\gamma)\right) \mid \gamma^{\prime} \in \Gamma\right\}:=[(p, \gamma)]$ of the action of $\Gamma$ on $P \times \Gamma$. Orbits $\left[\left(p, \gamma_{1}\right)\right]$ and $\left[\left(p, \gamma_{2}\right)\right]$ may be multiplied to $\left[\left(p, \gamma_{1} \gamma_{2}\right)\right]$ so that $A d(P)$ is a bundle of groups. Consequently, sections of $A d(P)$ may be multiplied point-wise, forming the group of gauge transformations. An orbit $[(p, \gamma)]$ acts (from the left) on the fiber $\pi^{-1}(\pi(p)) \subset P$ by the mapping

$$
p \gamma^{\prime} \longmapsto p \gamma \gamma^{\prime}=:[(p, \gamma)] \cdot p \gamma^{\prime}
$$

This action induces a left action of gauge transformations $h$ on $P$ by principal $\Gamma$-bundle automorphisms $\lambda_{h}$ given by

$$
P \ni p \stackrel{\lambda_{h}}{\longrightarrow} h(x) \cdot p
$$

Gauge transformations of the gauge field $\mathcal{A}$ are defined as

$$
\mathcal{A} \longmapsto h \mathcal{A}:=\lambda_{h^{-1}}^{*} \mathcal{A} .
$$

Note that the maps

$$
\tilde{L}_{h}:=\lambda_{h} \times I d
$$

from $\tilde{M}=P \times M$ into itself are $\Gamma$-equivariant, i.e. they commute with the action (5.9) of $\Gamma$ on $\tilde{M}$. Consequently, they descend to automorphisms $L_{h}$ of the associated bundle $P_{M}=P \times_{\Gamma} M$. Gauge transformations of sections $\Phi$ of $P_{M}$ are defined by the formula

$$
\Phi \longmapsto L_{h} \circ \Phi=: h \Phi .
$$

In the case of the trivial bundle $P$, the associated bundle $A d(P)$ is also trivial and the sections $h$ of $A d(P)$ reduce to maps from $\Sigma$ to $\Gamma$. Their action on gauge fields $\mathcal{A}$ agrees with the action (4.3) on the 1-forms $A$ related to $\mathcal{A}$ as in Remark 5.8. Similarly, their action on sections $\Phi$ of the trivial associated bundle agrees with the one considered in Eq. (4.2). The invariance of the amplitudes $\boldsymbol{A}_{W Z}(\Phi, \mathcal{A})$ from Definition 5.7 in the case of the trivial bundle $P$ is assured by the assumption of the $\Gamma$-equivariance of the gerbe $\mathcal{G}$. Indeed, as follows from Corollary 4.5, only property $(i)$ of Definition 5.1 is needed in that case to guarantee the gauge invariance under general gauge transformations. Here, we shall prove for a general principal $\Gamma$-bundles $P$,

Theorem 5.9. The amplitudes $\boldsymbol{A}_{W Z}(\Phi, \mathcal{A})$ of Definition 5.7 are invariant under all gauge transformations, i.e.

$$
\boldsymbol{A}_{W Z}(h \Phi, h \mathcal{A})=\boldsymbol{A}_{W Z}(\Phi, \mathcal{A})
$$

for all sections $h$ of the bundle Ad(P).

Proof. We have to show that

$$
\operatorname{Hol}_{\mathcal{G}_{h \mathcal{A}}}\left(L_{h} \circ \Phi\right)=\operatorname{Hol}_{L_{h}^{*} \mathcal{G}_{h \mathcal{A}}}(\Phi)=\operatorname{Hol}_{\mathcal{G}_{\mathcal{A}}}(\Phi)
$$

for all $h, \Phi$ and $\mathcal{A}$. This follows if there exists a 1-isomorphism between gerbes $L_{h}^{*} \mathcal{G}_{h \mathcal{A}}$ and $\mathcal{G}_{\mathcal{A}}$. Recall that gerbe $\mathcal{G}_{\mathcal{A}}$ over $P_{M}$ descended from the $\Gamma$-equivariant gerbe $\left(\tilde{\mathcal{G}}_{\mathcal{A}}, \tilde{\alpha}_{\mathcal{A}}, \tilde{\beta}_{\mathcal{A}}\right)$ over $\tilde{M}$, see Proposition 5.5 and Corollary 5.6. Since maps $\tilde{L}_{h}$ 
of $\tilde{M}$ are $\Gamma$-equivariant, gerbe $L_{h}^{*} \mathcal{G}_{A}$ descends, in turn, from the $\Gamma$-equivariant gerbe $\left(\tilde{L}_{h}^{*} \tilde{\mathcal{G}}_{\mathcal{A}},\left(\tilde{L}_{h}\right)_{\tilde{2}}^{*} \tilde{\alpha}_{\mathcal{A}},\left(\tilde{L}_{h}\right)_{\tilde{3}}^{*} \tilde{\beta}_{\mathcal{A}}\right)$, see Theorem 5.3. We claim that the two gerbes

$$
\left(\tilde{L}_{h}^{*} \tilde{\mathcal{G}}_{\mathcal{A}},\left(\tilde{L}_{h}\right)_{\tilde{2}}^{*} \tilde{\alpha}_{\mathcal{A}},\left(\tilde{L}_{h}\right)_{\tilde{3}}^{*} \tilde{\beta}_{\mathcal{A}}\right) \text { and }\left(\tilde{\mathcal{G}}_{h^{-1} \mathcal{A}}, \tilde{\alpha}_{h^{-1} \mathcal{A}}, \tilde{\beta}_{h^{-1} \mathcal{A}}\right)
$$

coincide. The claim implies, by virtue of Theorem 5.3, that the descended gerbes $L_{h}^{*} \mathcal{G}_{\mathcal{A}}$ and $\mathcal{G}_{h^{-1} \mathcal{A}}$ over $P_{M}$ coincide as well and, hence, so do $L_{h}^{*} \mathcal{G}_{h \mathcal{A}}$ and $\mathcal{G}_{\mathcal{A}}$.

It remains to prove the above claim. From definitions (5.8) of the form $\tilde{\rho}_{A},(5.26)$ of $h \mathcal{A}$ and (5.27) of the action $\tilde{L}_{h}$ on $\tilde{M}$, using, in particular, the fact that $\tilde{L}_{h}$ acts trivially on the factor $M$ in $P \times M$, it follows immediately that

$$
\tilde{L}_{h}^{*} \tilde{\rho}_{\mathcal{A}}=\tilde{\rho}_{h^{-1} \mathcal{A}}
$$

This, in conjunction with definition (5.11), implies, in turn, the equality of gerbes

$$
\tilde{L}_{h}^{*} \tilde{\mathcal{G}}_{\mathcal{A}}=\tilde{\mathcal{G}}_{h^{-1} \mathcal{A}}
$$

Recall from the proof of Proposition 5.5 that $\tilde{\alpha}_{\mathcal{A}}$ is the tensor product of the identity 1-isomorphism of the gerbe $\mathcal{I}_{\left(\tilde{\rho}_{\mathcal{A}}\right)_{\tilde{1} \tilde{2}}}$ with the 1-isomorphism $\alpha_{1,3}$. Now, the map $\left(\tilde{L}_{h}\right)_{2}$ of $\Gamma \times \tilde{M}=\Gamma \times P \times M$ acts only on the factor $P$. Besides,

$$
\left(\tilde{L}_{h}\right)_{\tilde{2}}^{*}\left(\tilde{\rho}_{\mathcal{A}}\right)_{\tilde{1} \tilde{2}}=\left(\tilde{L}_{h}^{*} \tilde{\rho}_{\mathcal{A}}\right)_{\tilde{1} \tilde{2}}=\left(\tilde{\rho}_{h^{-1} \mathcal{A}}\right)_{\tilde{1} \tilde{2}}
$$

We infer this way that the 1-isomorphism $\left(\tilde{L}_{h}\right)_{\tilde{2}}^{*} \tilde{\alpha}_{\mathcal{A}}$ is the tensor product of the identity 1 -isomorphism of the gerbe $\mathcal{I}_{\tilde{\rho}_{h^{-1} \mathcal{A}}}$ with the 1-isomorphism $\alpha_{1,3}$ so that

$$
\left(\tilde{L}_{h}\right)_{\tilde{2}}^{*} \tilde{\alpha}_{\mathcal{A}}=\tilde{\alpha}_{h^{-1} \mathcal{A}}
$$

Additionally, equalities (5.32) and (5.34) allow to relate the 2-isomorphisms $\left(\tilde{L}_{h}\right)_{\tilde{3}}^{*} \tilde{\beta}_{\mathcal{A}}$ and $\tilde{\beta}_{h^{-1} \mathcal{A}}$. Indeed, both are tensor products of the identity 2 -isomorphism between the identity 1-isomorphisms of the gerbe $\left.\left(\tilde{L}_{h}\right)_{\tilde{3}}^{*} \mathcal{I}_{\left(\tilde{\rho}_{\mathcal{A}}\right)_{\tilde{1} \tilde{2} \tilde{3}}}=\mathcal{I}_{\left(\tilde{\rho}_{h}-1 \mathcal{A}\right.}\right)_{\tilde{1} \tilde{2} \tilde{3}}$ with the 2 -isomorphism $\beta_{1,2,4}$, see Eq. (5.21). Hence,

$$
\left(\tilde{L}_{h}\right)_{\tilde{3}}^{*} \tilde{\beta}_{\mathcal{A}}=\tilde{\beta}_{h^{-1} \mathcal{A}},
$$

and the claim is established.

\section{Obstructions and Classification of Equivariant Structures}

In this section, we shall treat the obstructions to the existence and the classification of equivariant structures on gerbe $\mathcal{G}$ over a $\Gamma$-space, see Definition 5.1. We shall start by discussing subsequently the obstructions to the three parts of the structure: 1-isomorphism $\alpha, 2$-isomorphism $\beta$, and the commutative diagram (5.1). 
6.1. Obstructions to 1-isomorphisms $\alpha$. The first obstruction concerns the existence of 1-isomorphism $\alpha: \mathcal{G}_{12} \rightarrow \mathcal{I}_{\rho} \otimes \mathcal{G}_{2}$ or, equivalently, the triviality of 1-isomorphism class $[\mathcal{F}] \in H^{2}(\Gamma \times M, U(1))$ of the flat gerbe $\mathcal{F}=\mathcal{G}_{12} \otimes \mathcal{G}_{2}^{*} \otimes \mathcal{I}_{-\rho}$ over $\Gamma \times M$. It coincides with the obstruction to the general gauge invariance of the WZ amplitudes (3.10) coupled to topologically trivial gauge fields, see Corollary 4.5. By the Universal Coefficient Theorem, $H^{2}(\Gamma \times M, U(1))=H o m\left(H_{2}(\Gamma \times M), U(1)\right)$. In the latter presentation, class $[\mathcal{F}]$ is given by the holonomy of the flat gerbe $\mathcal{F}$ along maps $(h, \varphi): \Sigma \rightarrow \Gamma \times M$ defining singular 2-cycles, and its triviality is equivalent to the triviality of the holonomy. By the Künneth Theorem,

$$
H_{2}(\Gamma \times M)=H_{2}(\Gamma) \otimes H_{0}(M) \oplus H_{1}(\Gamma) \otimes H_{1}(M) \oplus H_{0}(\Gamma) \otimes H_{2}(M) .
$$

Subgroup $H_{2}(\Gamma) \otimes H_{0}(M) \cong H_{2}(\Gamma)^{\pi_{0}(M)}$ is generated by the singular 2-cycles corresponding to maps $(h, \varphi)$ with $\varphi$ taking a constant value in one of the connected components of $M\left(\pi_{0}(M)\right.$ is the set of such components). Similarly for $H_{0}(\Gamma) \otimes H_{2}(M) \cong$ $H_{2}(M)^{\pi_{0}(\Gamma)}$. Subgroup $H_{1}(\Gamma) \otimes H_{1}(M)$ is generated by the maps

$$
S^{1} \times S^{1} \ni\left(\mathrm{e}^{i \sigma_{1}}, \mathrm{e}^{i \sigma_{2}}\right) \longmapsto\left(h\left(\mathrm{e}^{i \sigma_{1}}\right), \varphi\left(\mathrm{e}^{i \sigma_{2}}\right)\right) \in \Gamma \times M
$$

with $h$ and $\varphi$ giving rise to singular 1-cycles in $\Gamma$ and $M$, respectively. Thus,

$$
\begin{aligned}
& H^{2}(\Gamma \times M, U(1)) \\
&= \operatorname{Hom}\left(H_{2}(\Gamma)^{\pi_{0}(M)}, U(1)\right) \oplus \operatorname{Hom}\left(H_{1}(\Gamma) \otimes H_{1}(M), U(1)\right) \\
& \oplus \operatorname{Hom}\left(H_{2}(M)^{\pi_{0}(\Gamma)}, U(1)\right) \\
&= H^{2}(\Gamma, U(1))^{\pi_{0}(M)} \oplus \operatorname{Hom}\left(H_{1}(\Gamma) \otimes H_{1}(M), U(1)\right) \oplus H^{2}(M, U(1))^{\pi_{0}(\Gamma)} .
\end{aligned}
$$

Accordingly, we obtain

Proposition 6.1. Class $[\mathcal{F}] \in H^{2}(\Gamma \times M, U(1))$ that obstructs the existence of 1 -isomorphism $\alpha$ of Definition 5.1 decomposes as

$$
[\mathcal{F}]=[\mathcal{F}]_{20}+[\mathcal{F}]_{11}+[\mathcal{F}]_{02},
$$

with the summands $[\mathcal{F}]_{20} \in H^{2}(\Gamma, U(1))^{\pi_{0}(M)},[\mathcal{F}]_{11} \in \operatorname{Hom}\left(H_{1}(\Gamma) \otimes H_{1}(M), U(1)\right)$ and $[\mathcal{F}]_{02} \in H^{2}(M, U(1))^{\pi_{0}(\Gamma)}$.

Components of $[\mathcal{F}]_{20}$ are the 1-isomorphism classes of flat gerbes $r_{m}^{*} \mathcal{G} \otimes \mathcal{I}_{-\rho_{m}}$ over $\Gamma$ for fixed points $m$ in different connected components of $M$ with $r_{m}(\gamma)=\gamma m=\ell_{\gamma}(m)$ and $\rho_{m}=\frac{1}{2}\left(\iota^{a} v^{b}\right)(m) \Theta^{a} \Theta^{b}$. Components of $[\mathcal{F}]_{02}$ are the 1-isomorphism classes of flat gerbes $\ell_{\gamma}^{*} \mathcal{G} \otimes \mathcal{G}^{*}$ for fixed points $\gamma$ in different connected components of $\Gamma$. Finally, the bihomomorphism $[\mathcal{F}]_{11} \in \operatorname{Hom}\left(H_{1}(\Gamma) \otimes H_{1}(M), U(1)\right)$ is given by the gerbe $\mathcal{F}$ holonomy of the maps (6.2).

Corollary 6.2. If the connected components of $M$ and $\Gamma$ are 2-connected, then there is no obstruction to the existence of 1-isomorphism $\alpha$ of Definition 5.1.

This applies to the case, studied in [26,27], of $\Gamma$-equivariant structures on the WZW gerbe $\mathcal{G}_{k}$ over $\tilde{G}$ for $\Gamma=Z \subset \tilde{Z}$ acting on $\tilde{G}$ by multiplication. 
For the $\Gamma$-space $M=G$ in the coset-model context, see Definition 4.6, and with a WZW gerbe $\mathcal{G}_{k}$ over $G$, the flat gerbe $\mathcal{F}$ was denoted $\mathcal{F}_{k}$, see Sect. 4.2. In decomposition (6.4) of cohomology class $\left[\mathcal{F}_{k}\right] \in H^{2}(\Gamma \times G, U(1))$, terms $\left[\mathcal{F}_{k}\right]_{20}$ and $\left[\mathcal{F}_{k}\right]_{02}$ are

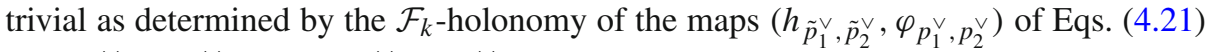
with $p_{1}^{\vee}=p_{2}^{\vee}=0$ or $\tilde{p}_{1}^{\vee}=\tilde{p}_{2}^{\vee}=0$, respectively, whereas the bihomomorphism $\left[\mathcal{F}_{k}\right]_{11} \in \operatorname{Hom}\left(\tilde{Z}_{\Gamma} \otimes Z, U(1)\right)$ is determined by the holonomy with $\tilde{p}_{2}^{\vee}=p_{1}^{\vee}=0$, i.e. by

$$
b_{\tilde{z}_{1}, z_{2}}=\exp \left[-2 \pi i k \operatorname{tr} \tilde{p}_{1}^{\vee} p_{2}^{\vee}\right],
$$

see Eq. (4.22), and may be non-trivial.

6.2. Local description of gerbes. In order to discuss further obstructions to the existence of a $\Gamma$-equivariant structure on gerbe $\mathcal{G}$ over $\Gamma$-space $M$, it will be convenient to use local data for gerbes and their 1- and 2-isomorphisms. We shall follow the discussion in the first part of Sec. VII of [25]. The local data live in the Deligne complex $\mathcal{D}(2)$

$$
0 \longrightarrow A^{0}(\mathcal{O}) \stackrel{D_{0}}{\longrightarrow} A^{1}(\mathcal{O}) \stackrel{D_{1}}{\longrightarrow} A^{2}(\mathcal{O}) \stackrel{D_{2}}{\longrightarrow} A^{3}(\mathcal{O})
$$

associated to an open covering $\mathcal{O}$ of $M$. With $\mathcal{U}$ standing for the sheaf of smooth $U(1)$ valued functions and $\Lambda^{n}$ for the sheaf of $n$-forms, the groups of the Deligne complex are

$$
\begin{aligned}
& A^{0}(\mathcal{O})=C^{0}(\mathcal{O}, \mathcal{U}), \\
& A^{1}(\mathcal{O})=C^{0}\left(\mathcal{O}, \Lambda^{1}\right) \oplus C^{1}(\mathcal{O}, \mathcal{U}), \\
& A^{2}(\mathcal{O})=C^{0}\left(\mathcal{O}, \Lambda^{2}\right) \oplus C^{1}\left(\mathcal{O}, \Lambda^{1}\right) \oplus C^{2}(\mathcal{O}, \mathcal{U}), \\
& A^{3}(\mathcal{O})=C^{1}\left(\mathcal{O}, \Lambda^{2}\right) \oplus C^{2}\left(\mathcal{O}, \Lambda^{1}\right) \oplus C^{3}(\mathcal{O}, \mathcal{U}),
\end{aligned}
$$

where $C^{\ell}(\mathcal{O}, \mathcal{S})$ denotes the $\ell^{\text {th }}$ Čech cochain group of the open cover $\mathcal{O}$, with values in a sheaf $\mathcal{S}$ of Abelian groups. The differentials are

$$
\begin{aligned}
& D_{0}\left(f_{i}\right)=\left(-\mathrm{i} f_{i}^{-1} d f_{i}, f_{j}^{-1} f_{i}\right), \quad D_{1}\left(\Pi_{i}, \chi_{i j}\right) \\
& \quad=\left(d \Pi_{i},-\mathrm{i} \chi_{i j}^{-1} d \chi_{i j}+\Pi_{j}-\Pi_{i}, \chi_{j k}^{-1} \chi_{i k} \chi_{i j}^{-1}\right), \\
& D_{2}\left(B_{i}, A_{i j}, g_{i j k}\right) \\
& \quad=\left(d A_{i j}-B_{j}+B_{i},-\mathrm{i} g_{i j k}^{-1} d g_{i j k}+A_{j k}-A_{i k}+A_{i j}, g_{j k l}^{-1} g_{i k l} g_{i j l}^{-1} g_{i j k}\right) .
\end{aligned}
$$

A refinement $r: \mathcal{O}^{\prime} \rightarrow \mathcal{O}$ of covering $\mathcal{O}$ induces a restriction map on complexes (6.6). Local data for gerbe $\mathcal{G}$ over $M$ form a cocycle $c \in A^{2}(\mathcal{O}), D_{2} c=0$, for a sufficiently fine covering $\mathcal{O}$ of $M$. Local data for 1-isomorphism $\alpha: \mathcal{G}_{1} \rightarrow \mathcal{G}_{2}$ of gerbes with the respective local data $c_{i} \in A^{2}\left(\mathcal{O}_{i}\right)$ are given by a cochain $b \in A^{1}(\mathcal{O})$ for $\mathcal{O}$ a common refinement of $\mathcal{O}_{1}$ and $\mathcal{O}_{2}$ such that, upon restricting the $c_{i}$ to it, $c_{2}=c_{1}+D_{1} b$ (we use the additive notation for the Abelian group law in all $A^{n}(\mathcal{O})$ ). Finally, local data for 2-isomorphism $\beta: \alpha_{1} \Rightarrow \alpha_{2}$ are given by a cochain $a \in A^{0}(\mathcal{O})$ for a sufficiently fine covering $\mathcal{O}$ such that, given local data $b_{i}$ for 1-isomorphisms $\alpha_{i}$ restricted to $\mathcal{O}, b_{2}=b_{1}+D_{0} a$. For sufficiently fine $\mathcal{O}$, the cohomology of the complex (6.6) is

$$
\begin{aligned}
\mathbb{H}^{2}(\mathcal{O}, \mathcal{D}(2))= & \frac{\operatorname{ker} D_{2}}{\operatorname{Im} D_{1}}, \quad \mathbb{H}^{1}(\mathcal{O}, \mathcal{D}(2))=\frac{\operatorname{ker} D_{1}}{\operatorname{Im} D_{0}} \cong H^{1}(M, U(1)), \\
& \mathbb{H}^{0}(\mathcal{O}, \mathcal{D}(2))=\operatorname{ker} D_{0} \cong H^{0}(M, U(1)) .
\end{aligned}
$$


These groups may be identified, respectively, with the group of 1-isomorphism classes of gerbes, the group of isomorphism classes of flat line bundles, and the group of locally constant $U(1)$-valued functions on $M$.

In the following, we want to consider local data for gerbes and their 1- and 2-isomorphisms over the spaces $\Gamma^{p} \times M$ that form a simplicial manifold with face maps $\Delta_{q}^{p}: \Gamma^{p} \times M \rightarrow \Gamma^{p-1} \times M$ for all $p \geq 1$ and $0 \leq q \leq p$ given by

$$
\Delta_{q}^{p}\left(\gamma_{1}, \ldots, \gamma_{p}, m\right):= \begin{cases}\left(\gamma_{2}, \ldots, \gamma_{p}, m\right) & \text { for } q=0 \\ \left(\gamma_{1}, \ldots, \gamma_{q} \gamma_{q+1}, \ldots, \gamma_{p}, m\right) & \text { for } 1 \leq q<p \\ \left(\gamma_{1}, \ldots, \gamma_{p-1}, \gamma_{p} m\right) & \text { for } q=p\end{cases}
$$

The face maps satisfy the simplicial relations

$$
\Delta_{r}^{p-1} \circ \Delta_{q}^{p}=\Delta_{q-1}^{p-1} \circ \Delta_{r}^{p}
$$

for all $r<q$. We shall use simplicial sequences $\left\{\mathcal{O}^{p}\right\}$ of open coverings $\mathcal{O}^{p}=\left\{O_{i}^{p}\right\}_{i \in I^{p}}$ of the spaces $\Gamma^{p} \times M$ such that there are face maps $\Delta_{q}^{p}: I^{p} \rightarrow I^{p-1}$ of the index sets satisfying (6.14), and such that

$$
\Delta_{q}^{p}\left(O_{i}^{p}\right) \subset O_{\Delta_{q}^{p}(i)}^{p-1}
$$

for all $p \geq 1$, all $0 \leq q \leq p$ and all $i \in I^{p}$. A construction of Ref. [52], reviewed in the Appendix of [25], permits to build a simplicial sequence $\left\{\mathcal{O}^{p}\right\}$ whose coverings $\mathcal{O}^{p}$ refine the coverings of any given sequence of coverings of $\Gamma^{p} \times M$. Given a simplicial sequence $\left\{\mathcal{O}^{p}\right\}$ of coverings of $\Gamma^{p} \times M$, one has induced cochain maps

$$
\left(\Delta_{q}^{p}\right)^{*}: C^{\ell}\left(\mathcal{O}^{p-1}, \mathcal{S}\right) \rightarrow C^{\ell}\left(\mathcal{O}^{p}, \mathcal{S}\right) \text { defined by }\left(\left(\Delta_{q}^{p}\right)^{*} f\right)_{i}=\left(\Delta_{q}^{p}\right)^{*}\left(f_{\Delta_{q}^{p}(i)}\right)
$$

satisfying the co-simplicial relations

$$
\left(\Delta_{q}^{p}\right)^{*} \circ\left(\Delta_{r}^{p-1}\right)^{*}=\left(\Delta_{r}^{p}\right)^{*} \circ\left(\Delta_{q-1}^{p-1}\right)^{*}
$$

for $r<q$. On the groups $A^{n}\left(\mathcal{O}^{p}\right)$, besides the Deligne differentials

$$
D_{n, p}: A^{n}\left(\mathcal{O}^{p}\right) \rightarrow A^{n+1}\left(\mathcal{O}^{p}\right)
$$

one has the simplicial operators

$$
\Delta_{n, p}: A^{n}\left(\mathcal{O}^{p}\right) \rightarrow A^{n}\left(\mathcal{O}^{p+1}\right) \quad \text { with } \quad \Delta_{n, p}:=\sum_{q=0}^{p+1}(-1)^{q}\left(\Delta_{q}^{p+1}\right)^{*}
$$

whose definition uses the lift (6.16) of the face maps to groups $A^{n}\left(\mathcal{O}^{p}\right)$. Due to the co-simplicial relations (6.17), we have $\Delta_{n, p+1} \circ \Delta_{n, p}=0$. The differentials $D_{n, p}$ commute with pullbacks, and thus also with operators $\Delta_{n, p}$. This endows the family $\mathcal{K}=$ $\left(A^{n}\left(\mathcal{O}^{p}\right)\right)$ of Abelian groups with the structure of a double complex. 
6.3. Obstructions to 2-isomorphism $\beta$. If cocycle $c \in A^{2}\left(\mathcal{O}^{0}\right)$ describes local data for gerbe $\mathcal{G}$ over $M$ then $-\left(\Delta_{2,0} c+\rho\right) \in A^{2}\left(\mathcal{O}^{1}\right)$, where $\rho$ is identified with the cochain $\left(\left.\rho\right|_{O_{i}^{1}}, 0,1\right)$ for $i \in I^{1}$, represents local data for the flat gerbe $\mathcal{F}=\mathcal{G}_{12} \otimes \mathcal{G}_{2}^{*} \otimes \mathcal{I}_{-\rho}$. The triviality of 1-isomorphism class $[\mathcal{F}]$, discussed in Sect. 6.1, means that, for a sufficiently fine simplicial sequence of coverings $\left\{\mathcal{O}^{p}\right\}$,

$$
\Delta_{2,0} c+\rho=D_{1,1} b
$$

for some $b \in A^{1}\left(\mathcal{O}^{1}\right)$. The cochain $b$ provides local data for a 1-isomorphism $\alpha$ : $\mathcal{G}_{12} \rightarrow \mathcal{I}_{\rho} \otimes \mathcal{G}_{2}$, see Definition 5.1. It is defined modulo the addition $b \mapsto b+b^{\prime}$, where $D_{1,1} b^{\prime}=0$. This freedom corresponds to the freedom of choice of $\alpha$ and of local data for it. The cochains $\left(\Delta_{0}^{2}\right)^{*} b,\left(\Delta_{1}^{2}\right)^{*} b$ and $\left(\Delta_{2}^{2}\right)^{*} b$ provide, in turn, local data for 1-isomorphisms $\alpha_{2,3}, \alpha_{12,3}$ and $\alpha_{1,23}$, respectively. The existence of 2 -isomorphism $\beta:\left(I d \otimes \alpha_{2,3}\right) \circ \alpha_{1,23} \Rightarrow \alpha_{12,3}$ is equivalent to the requirement that, for sufficiently fine $\left\{\mathcal{O}^{p}\right\}$,

$$
\Delta_{1,1} b=-D_{0,2} a
$$

with $a \in A^{0}\left(\mathcal{O}^{2}\right)$ representing local data for $\beta$. Let us first note that

$$
D_{1,2} \Delta_{1,1} b=\Delta_{2,1} D_{1,1} b=\Delta_{2,1} \Delta_{2,0} c+\Delta_{2,1} \rho=0
$$

where the last equality is a consequence of relations $\Delta_{2,1} \circ \Delta_{2,0}=0$ and $\Delta_{2,1} \rho=$ $\left(\left.\left(\rho_{2,3}-\rho_{12,3}+\rho_{1,23}\right)\right|_{O_{i}^{2}}, 0,0\right)$, and of Eq. (3.31) of Lemma 3.13. It follows that $\Delta_{1,1} b$ defines a cohomology class

$$
\left[\Delta_{1,1} b\right] \in \frac{\operatorname{ker} D_{1,2}}{\operatorname{Im} D_{0,2}} \cong H^{1}\left(\Gamma^{2} \times M, U(1)\right)
$$

that obstructs the solution of Eq. (6.21). However, since $b$ was defined up to $D_{1,1}$-cocycles $b^{\prime} \in A^{1}\left(\mathcal{O}^{1}\right)$, the class $\left[\Delta_{1,1} b\right]$ is defined modulo the image $\mathcal{H}^{1,2}$ of the map $\left[\Delta_{1,1}\right]: H^{1}(\Gamma \times M, U(1)) \rightarrow H^{1}\left(\Gamma^{2} \times M, U(1)\right)$ that sends class [ $\left.b^{\prime}\right]$ to class $\left[\Delta_{1,1} b^{\prime}\right]$. We obtain this way

Proposition 6.3. Let $\alpha: \mathcal{G}_{12} \rightarrow \mathcal{I}_{\rho} \otimes \mathcal{G}_{2}$ be a 1-isomorphism with local data $b \in$ $A^{1}\left(\mathcal{O}^{1}\right)$ for a sufficiently fine family of coverings $\left\{\mathcal{O}^{p}\right\}$. Then there exists 2-isomorphism $\beta$ for a, possibly modified, choice of 1-isomorphism $\alpha$ if and only if the obstruction class

$$
\left[\Delta_{1,1} b\right]+\mathcal{H}^{1,2} \in H^{1}\left(\Gamma^{2} \times M, U(1)\right) / \mathcal{H}^{1,2}
$$

vanishes.

In the particular case with simply connected components of $\Gamma$ and $M$, groups $H^{1}\left(\Gamma^{p} \times M, U(1)\right)$ are trivial and we obtain

Corollary 6.4. If the connected components of $\Gamma$ and $M$ are simply connected then the class (6.24) obstructing the existence of 2-isomorphism $\beta$ is trivial. 
This applies to the case of $Z$-equivariant structures on gerbes $\mathcal{G}_{k}$ over groups $\tilde{G}$ discussed in [26,27].

In the general situation, a more precise description of spaces $H^{1}\left(\Gamma^{2} \times M, U(1)\right) \supset$ $\mathcal{H}^{1,2}$ may be provided with the help of the Universal Coefficient and Künneth Theorems. One has

$$
H^{1}(\Gamma \times M, U(1)) \cong H^{1}(\Gamma, U(1))^{\pi_{0}(M)} \oplus H^{1}(M, U(1))^{\pi_{0}(\Gamma)} .
$$

The element $\left[b^{\prime}\right] \in H^{1}(\Gamma \times M, U(1))$ is represented by the sequences with elements

$$
\left[b^{\prime}\right]_{1}([m]):=\left(\iota_{m}^{1}\right)^{*}\left[b^{\prime}\right] \in H^{1}(\Gamma, U(1)), \quad\left[b^{\prime}\right]_{2}([\gamma]):=\left(\iota_{\gamma}^{2}\right)^{*}\left[b^{\prime}\right] \in H^{1}(M, U(1)),
$$

where $m$, resp. $\gamma$, are chosen points in the connected components $[m] \in \pi_{0}(M)$, resp. $[\gamma] \in \pi_{0}(\Gamma)$, and $\iota_{m}^{1}: \Gamma \rightarrow \Gamma \times M$, resp. $\iota_{\gamma}^{2}: M \rightarrow \Gamma \times M$, are the injections with $\iota_{m}^{1}(\gamma)=\iota_{\gamma}^{2}(m)=(\gamma, m)$. Similarly,

$$
\begin{aligned}
& H^{1}\left(\Gamma^{2} \times M, U(1)\right) \\
& \cong H^{1}(\Gamma, U(1))^{\pi_{0}(\Gamma) \times \pi_{0}(M)} \oplus H^{1}(\Gamma, U(1))^{\pi_{0}(\Gamma) \times \pi_{0}(M)} \oplus H^{1}(M, U(1))^{\pi_{0}(\Gamma)^{2}} .
\end{aligned}
$$

An element $[d] \in H^{1}\left(\Gamma^{2} \times M, U(1)\right)$ is represented by the sequences with elements

$$
\begin{aligned}
& {[d]_{1}\left(\left[\gamma_{2}\right],[m]\right):=\left(\iota_{\gamma_{2}, m}^{1}\right)^{*}[d] \in H^{1}(\Gamma, U(1)),} \\
& {[d]_{2}\left(\left[\gamma_{1}\right],[m]\right):=\left(\iota_{\gamma_{1}, m}^{2}\right)^{*}[d] \in H^{1}(\Gamma, U(1)),} \\
& {[d]_{3}\left(\left[\gamma_{1}\right],\left[\gamma_{2}\right]\right):=\left(\iota_{\gamma_{1}, \gamma_{2}}^{3}\right)^{*}[d] \in H^{1}(M, U(1)),}
\end{aligned}
$$

where $\iota_{\gamma_{2}, m}^{1}, \iota_{\gamma_{1}, m}^{2}: \Gamma \rightarrow \Gamma^{2} \times M$ and $\iota_{\gamma_{1}, \gamma_{2}}^{3}: M \rightarrow \Gamma^{2} \times M$ are the injections with $\iota_{\gamma_{2}, m}^{1}\left(\gamma_{1}\right)=\iota_{\gamma_{1}, m}^{2}\left(\gamma_{2}\right)=\iota_{\gamma_{1}, \gamma_{2}}^{3}(m)=\left(\gamma_{1}, \gamma_{2}, m\right)$. Compositions of the above injections with simplicial maps $\Delta_{q}^{2}$ are

$$
\begin{aligned}
& \Delta_{0}^{2} \circ \iota_{\gamma_{2}, m}^{1}\left(\gamma_{1}\right)=\Delta_{0}^{2} \circ \iota_{\gamma_{1}, m}^{2}\left(\gamma_{2}\right)=\Delta_{0}^{2} \circ \iota_{\gamma_{1}, \gamma_{2}}^{3}(m)=\left(\gamma_{2}, m\right), \\
& \Delta_{1}^{2} \circ \iota_{\gamma_{2}, m}^{1}\left(\gamma_{1}\right)=\Delta_{1}^{2} \circ \iota_{\gamma_{1}, m}^{2}\left(\gamma_{2}\right)=\Delta_{1}^{2} \circ \iota_{\gamma_{1}, \gamma_{2}}^{3}(m)=\left(\gamma_{1} \gamma_{2}, m\right), \\
& \Delta_{2}^{2} \circ \iota_{\gamma_{2}, m}^{1}\left(\gamma_{1}\right)=\Delta_{2}^{2} \circ \iota_{\gamma_{1}, m}^{2}\left(\gamma_{2}\right)=\Delta_{2}^{2} \circ \iota_{\gamma_{1}, \gamma_{2}}^{3}(m)=\left(\gamma_{1}, \gamma_{2} m\right) .
\end{aligned}
$$

Since $\Delta_{1,1}=\left(\Delta_{0}^{2}\right)^{*}-\left(\Delta_{1}^{2}\right)^{*}+\left(\Delta_{2}^{2}\right)^{*}$, it follows that

$$
\begin{aligned}
& {\left[\Delta_{1,1} b\right]_{1}\left(\left[\gamma_{2}\right],[m]\right)=\left[-R_{\gamma_{2}}^{*}\left(\iota_{m}^{1}\right)^{*} b+\left(\iota_{\gamma_{2} m}^{1}\right)^{*} b\right],} \\
& {\left[\Delta_{1,1} b\right]_{2}\left(\left[\gamma_{1}\right],[m]\right)=\left[\left(\iota_{m}^{1}\right)^{*} b-L_{\gamma_{1}}^{*}\left(\iota_{m}^{1}\right)^{*} b+\left(\iota_{\gamma_{1}}^{2} \circ r_{m}\right)^{*} b\right],} \\
& {\left[\Delta_{1,1} b\right]_{3}\left(\left[\gamma_{1}\right],\left[\gamma_{2}\right]\right)=\left[\left(\iota_{\gamma_{2}}^{2}\right)^{*} b-\left(\iota_{\gamma_{1} \gamma_{2}}^{2}\right)^{*} b+\ell_{\gamma_{2}}^{*}\left(\iota_{\gamma_{1}}^{2}\right)^{*} b\right],}
\end{aligned}
$$

where $L_{\gamma}, R_{\gamma}: \Gamma \rightarrow \Gamma$ denote, respectively, the left and the right multiplication by $\gamma, r_{m}(\gamma)=\gamma m$ (as before), and we used the fact that the class in $H^{1}(\Gamma, U(1))$ of the pullback of $A^{1}\left(\mathcal{O}^{1}\right)$ along a constant map is trivial. When the group $\Gamma$ is connected, we may choose its identity element as its special point and the above equations reduce to

$$
\begin{aligned}
& {\left[\Delta_{1,1} b\right]_{1}([1],[m])=0, \quad\left[\Delta_{1,1} b\right]_{2}([1],[m])=\left[\left(\iota_{1}^{2} \circ r_{m}\right)^{*} b\right],} \\
& {\left[\Delta_{1,1} b\right]_{3}([1],[1])=\left[\left(\iota_{1}^{2}\right)^{*} b\right] .}
\end{aligned}
$$


In the case of a $\Gamma$-space $M=G$ in the coset-model context of Definition 4.6, we may take $m=1 \in G$ in the last formulae which reduce then further to the relations

$$
\left[\Delta_{1,1} b\right]_{1}([1],[1])=0, \quad\left[\Delta_{1,1} b\right]_{2}([1],[1])=0, \quad\left[\Delta_{1,1} b\right]_{3}([1],[1])=\left[\left(\iota_{1}^{2}\right)^{*} b\right],
$$

because $\iota_{1}^{2} \circ r_{1}$ is a constant map. In particular, for $b=b^{\prime}$ with $D_{1,1} b^{\prime}=0$,

$$
\left[\Delta_{1,1} b^{\prime}\right]_{1}([1],[1])=0, \quad\left[\Delta_{1,1} b^{\prime}\right]_{2}([1],[1])=0, \quad\left[\Delta_{1,1} b^{\prime}\right]_{3}([1],[1])=\left[b^{\prime}\right]_{2}([1]) .
$$

Since $\left[b^{\prime}\right]_{2}([1])$ runs through arbitrary elements of $H^{1}(M, U(1))$, it follows that the obstruction class (6.24) vanishes and, for an appropriate choice of $b^{\prime}$ with $D_{1,1} b^{\prime}=0$, one has $\left[\Delta_{1,1}\left(b+b^{\prime}\right)\right]=0$ so that $\Delta_{1,1}\left(b+b^{\prime}\right)=-D_{0,2} a$ for some $a \in A^{0}\left(\mathcal{O}^{2}\right)$. We obtain this way

Corollary 6.5. For the $\Gamma$-space $M=G$ in the coset-model context, an appropriate choice of 1-isomorphism $\alpha$ of Definition 5.1 assures the existence of 2-isomorphism $\beta$.

6.4. Obstructions to the commutativity of diagram (5.1). By Proposition 6.3, the vanishing of obstruction (6.24) guarantees in the general case that 2-isomorphism $\beta$ exists for a suitable choice of 1 -isomorphism $\alpha$. In terms of local data, the condition $\left[\Delta_{1,1} b\right] \in \mathcal{H}^{1,2}$ assures that after a modification of local data $b$ by an appropriate $D_{1,1}$-cocycle $b^{\prime}$, determined up to the change $b^{\prime} \mapsto b^{\prime}-D_{0,1} a^{\prime \prime}$, there exists $a \in A^{0}\left(\mathcal{O}^{2}\right)$ such that

$$
\Delta_{1,1}\left(b+b^{\prime}\right)=-D_{0,2} a .
$$

In view of the freedom of choice of $b^{\prime}$, the cochain $a$ is determined up to the replacement $a \mapsto a+\Delta_{0,1} a^{\prime \prime}+a^{\prime \prime \prime}$ for $a^{\prime \prime} \in A^{0}\left(\mathcal{O}^{1}\right)$ and $a^{\prime \prime \prime} \in \operatorname{ker} D_{0,2} \cong H^{0}\left(\Gamma^{2} \times M, U(1)\right)$. Cocycle $a^{\prime \prime \prime}$ describes the possible choices of 2-isomorphism $\beta$. The commutativity of the diagram (5.1) of 2-isomorphisms of gerbes over $\Gamma^{3} \times M$ is now equivalent to the condition that, after the restriction to a sufficiently fine simplicial sequence of coverings,

$$
\Delta_{0,2} a=0 .
$$

Note that, in any case,

$$
D_{0,3} \Delta_{0,2} a=\Delta_{1,2} D_{0,2} a=-\Delta_{1,2} \Delta_{1,1}\left(b+b^{\prime}\right)=0
$$

so that $\Delta_{0,2} a \in \operatorname{ker} D_{0,3} \cong H^{0}\left(\Gamma^{3} \times M, U(1)\right)$. Let us denote by $\mathcal{H}^{0,3}$ the image of the map $\left[\Delta_{0,2}\right]: H^{0}\left(\Gamma^{2} \times M, U(1)\right) \rightarrow H^{0}\left(\Gamma^{3} \times M, U(1)\right)$ that sends $a^{\prime \prime \prime}$ to $\Delta_{0,2} a^{\prime \prime \prime}$. Using the freedom in the choice of the cochain $a$ and the relation

$$
\Delta_{0,2}\left(a+\Delta_{0,1} a^{\prime \prime}+a^{\prime \prime \prime}\right)=\Delta_{0,2} a+\Delta_{0,2} a^{\prime \prime \prime},
$$

we infer

Proposition 6.6. 2-isomorphism $\beta$ may be chosen so that the diagram (5.1) of Definition 5.1 is commutative if and only if the obstruction class

$$
\Delta_{0,2} a+\mathcal{H}^{0,3} \in H^{0}\left(\Gamma^{3} \times M, U(1)\right) / \mathcal{H}^{0,3}
$$

vanishes. 
Elements $f^{p} \in H^{0}\left(\Gamma^{p} \times M, U(1)\right)$ are locally constant $U(1)$-valued functions on $\Gamma^{p} \times M$. One may identify them with $p$-chains $v^{p}$ on the group $\pi_{0}(\Gamma)$ with values in the $\pi_{0}(\Gamma)$-module $U(1)^{\pi_{0}(M)} \cong H^{0}(M, U(1))$ of $U(1)$-valued functions on $\pi_{0}(M)$, where the action of $\pi_{0}(\Gamma)$ on $U(1)^{\pi_{0}(M)}$ is induced from the action of $\Gamma$ on $M$. If the identification is done by the formula

$$
f^{p}\left(\gamma_{1}, \ldots, \gamma_{p}, m\right)=v_{\left[\gamma_{p}^{-1}\right], \ldots,\left[\gamma_{1}^{-1}\right]}^{p}([m]),
$$

then the induced maps $\left[\Delta_{0, p}\right]: H^{0}\left(\Gamma^{p} \times M, U(1)\right) \rightarrow H^{0}\left(\Gamma^{p+1} \times M, U(1)\right)$ become the coboundary operators $\delta^{p}$ of the group $\pi_{0}(\Gamma)$ cohomology:

$$
\left(\Delta_{0, p} f^{p}\right)\left(\gamma_{1}, \ldots, \gamma_{p}, \gamma_{p+1}, m\right)=(-1)^{p+1}\left(\delta^{p} v^{p}\right)_{\left[\gamma_{p+1}^{-1}\right],\left[\gamma_{p}^{-1}\right], \ldots,\left[\gamma_{1}^{-1}\right]}([m]) .
$$

Corollary 6.7. Under identification (6.45), the cochain $\Delta_{0,2}$ a generates a 3-cocycle $v^{3}$ of the group $\pi_{0}(\Gamma)$ taking values in $U(1)^{\pi_{0}(M)}$ and the obstruction coset $(6.44)$ is the cohomology class $\left[v^{3}\right] \in H^{3}\left(\pi_{0}(\Gamma), U(1)^{\pi_{0}(M)}\right)$.

In particular, when $\Gamma$ is discrete and $M$ is connected, then $\left[v^{3}\right] \in H^{3}(\Gamma, U(1))$. That is the situation for the $Z$-equivariant structures on gerbes $\mathcal{G}_{k}$ over groups $\tilde{G}$ discussed in $[26,27]$ and mentioned already above. The obstruction cohomology classes $\left[v^{3}\right] \in H^{3}(Z, U(1))$ were computed for these cases and simple $\tilde{G}$ in [24].

Since the cohomology groups $H^{p}\left(\pi_{0}(\Gamma), U(1)^{\pi_{0}(M)}\right)$ for $p>1$ are trivial if $\pi_{0}(\Gamma)$ is a trivial group, we obtain

Corollary 6.8. If the symmetry group $\Gamma$ is connected and 2-isomorphism $\beta$ of Definition 5.1 exists, then it may always be chosen so that the diagram (5.1) commutes.

Putting together Proposition 4.8 and Corollaries 6.5 and 6.8, we summarize the results for the situation discussed in Sect. 4.2:

Theorem 6.9. For a $\Gamma$-space $M=G$ in the coset-model context of Definition 4.6, $a$ $\Gamma$-equivariant structure on the WZW gerbe $\mathcal{G}_{k}$ over $G$ exists if and only if the globalanomaly phases (4.22) are trivial, as, e.g., for $G=\tilde{G}$.

6.5. Classification of equivariant structures. Suppose now that we are given two equivariant structures $\left(\alpha_{i}, \beta_{i}\right), i=1,2$, on gerbe $\mathcal{G}$ with local data $c \in A^{2}\left(\mathcal{O}^{0}\right), D_{2,0} c=0$, for a sufficiently fine simplicial sequence of coverings $\left\{\mathcal{O}^{p}\right\}$. Their local data are $\left(b_{i}, a_{i}\right)$, with $b_{i} \in A^{1}\left(\mathcal{O}^{1}\right)$ and $a_{i} \in A^{0}\left(\mathcal{O}^{2}\right)$, that satisfy

$$
\Delta_{2,0} c+\rho=D_{1,1} b_{i}, \quad \Delta_{1,1} b_{i}=-D_{0,2} a_{i}, \quad \Delta_{0,2} a_{i}=0 .
$$

The difference $\left(b^{\prime}, a^{\prime}\right)=\left(b_{2}-b_{1}, a_{2}-a_{1}\right)$ gives local data for a $\Gamma$-equivariant structure on the trivial gerbe $\mathcal{I}_{0}$ (relative to $\rho=0$ ). It satisfies the homogeneous equations

$$
D_{1,1} b^{\prime}=0, \quad \Delta_{1,1} b^{\prime}=-D_{0,2} a^{\prime}, \quad \Delta_{0,2} a^{\prime}=0 .
$$

There is an isomorphism $(\chi, \eta)$ between the equivariant structures $\left(\alpha_{i}, \beta_{i}\right)$ if there exist: a cocycle $e \in A^{1}\left(\mathcal{O}^{0}\right), D_{1,0} e=0$ (providing local data for 1-isomorphism $\chi: \mathcal{G} \rightarrow \mathcal{G}$ ) and a cochain $f \in A^{0}\left(\mathcal{O}^{1}\right)$ (giving local data for 2-isomorphism $\eta$ ) such that

$$
b^{\prime}=\Delta_{1,0} e+D_{0,1} f, \quad a^{\prime}=-\Delta_{0,1} f .
$$


These identities represent the definition of $\eta$ and the commutativity of diagram (5.3), respectively. They imply Eqs. (6.48). Classes of solutions to Eqs. (6.48) modulo solutions to Eqs. (6.49) form the $2^{\text {nd }}$ hypercohomology group $\mathbb{H}^{2}(\mathcal{J})$ of the double complex $\mathcal{J}$

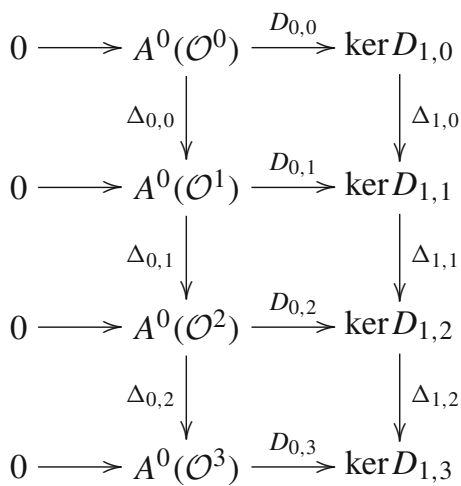

obtained from the double complex $\mathcal{K}=\left(A^{n}\left(\mathcal{O}^{p}\right)\right)$. $\mathbb{H}^{2}(\mathcal{J})$ is the group of isomorphism classes of $\Gamma$-equivariant structures on the trivial gerbe $\mathcal{I}_{0}$. It acts freely and transitively on the set of isomorphism classes of $\Gamma$-equivariant structures on gerbe $\mathcal{G}$. In other words,

Proposition 6.10. The set of isomorphisms classes of $\Gamma$-equivariant structures on gerbe $\mathcal{G}$ is a torsor for the Abelian group $\mathbb{H}^{2}(\mathcal{J})$.

Denote by $\mathcal{H}^{1,1}$ the image of the map $\left[\Delta_{1,0}\right]: H^{1}(M, U(1)) \rightarrow H^{1}(\Gamma \times M, U(1))$ that sends class $[e]$ to class $\left[\Delta_{1,0} e\right]$. In terms of the decomposition (6.25) and (6.26),

$$
\left[\Delta_{1,0} e\right]_{1}([m])=-\left[r_{m}^{*} e\right], \quad\left[\Delta_{1,0} e\right]_{2}([\gamma])=[e]-\left[\ell_{\gamma}^{*} e\right] .
$$

Since $b^{\prime}$ is a $D_{1,1}$-cocycle, one may consider the map

$$
\left(b^{\prime}, a^{\prime}\right) \longmapsto\left[b^{\prime}\right]+\mathcal{H}^{1,1} \in H^{1}(\Gamma \times M, U(1)) / \mathcal{H}^{1,1} .
$$

Since $\left[b^{\prime}\right] \in \mathcal{H}^{1,1}$ for $\left(b^{\prime}, a^{\prime}\right)$ of the form (6.49), the map (6.52) induces a homomorphism

$$
\kappa: \mathbb{H}^{2}(\mathcal{J}) \rightarrow H^{1}(\Gamma \times M, U(1)) / \mathcal{H}^{1,1}
$$

of Abelian groups. To describe the image and the kernel of $\kappa$, we shall do some tracing of diagrams.

If $\left[b^{\prime \prime}\right]+\mathcal{H}^{1,1}$ is in the image of $\kappa$, then $b^{\prime \prime}=b^{\prime}+\Delta_{1,0} e+D_{0,1} f$ for some $\left(b^{\prime}, a^{\prime}\right)$ as above, some $e \in A^{1}\left(\mathcal{O}^{0}\right)$ with $D_{1,0} e=0$, and some $f \in A^{0}\left(\mathcal{O}^{1}\right)$. Consequently, $\Delta_{1,1} b^{\prime \prime}=-D_{0,2} a^{\prime}+\Delta_{1,1} D_{0,1} f=-D_{0,2}\left(a^{\prime}-\Delta_{0,1} f\right)$ so that $\left[\Delta_{1,1}\right]\left[b^{\prime \prime}\right]=0$. For any $\left[b^{\prime \prime}\right]$ that satisfies the latter equation, i.e. such that $\Delta_{1,1} b^{\prime \prime}=-D_{0,2} a^{\prime \prime}$ for some $a^{\prime \prime} \in$ $A^{0}\left(\mathcal{O}^{2}\right)$, we have $D_{0,3} \Delta_{0,2} a^{\prime \prime}=\Delta_{1,2} D_{0,2} a^{\prime \prime}=0$, hence $\Delta_{0,2} a^{\prime \prime} \in H^{0}\left(\Gamma^{3} \times M, U(1)\right)$ and it generates, via Eq. (6.45), a 3-cocycle $v^{3}$ on group $\pi_{0}(\Gamma)$ with values in $U(1)^{\pi_{0}(M)}$. If $\left[b^{\prime \prime}\right]+\mathcal{H}^{1,1}$ is in the image of $\kappa$, then, for $a^{\prime \prime \prime}:=a^{\prime}-\Delta_{0,1} f-a^{\prime \prime}$, we have $D_{0,2} a^{\prime \prime \prime}=0$ so that $a^{\prime \prime \prime} \in H^{0}\left(\Gamma^{2} \times M, U(1)\right)$ generates, again via Eq. (6.45), a 2-cochain $u^{2}$ on $\pi_{0}(\Gamma)$ with values in $U(1)^{\pi_{0}(M)}$. The relation $\Delta_{0,2} a^{\prime \prime \prime}=-\Delta_{0,2} a^{\prime \prime}$ implies then that $\delta^{2} u^{2}=v^{3}$ so that the cohomology class of $\left[v^{3}\right] \in H^{3}\left(\pi_{0}(\Gamma), U(1)^{\pi_{0}(M)}\right)$ vanishes. Conversely, if this is the case, then $\Delta_{0,2} a^{\prime \prime}=-\Delta_{0,2} a^{\prime \prime \prime}$ for some $a^{\prime \prime \prime} \in H^{0}\left(\Gamma^{2} \times M, U(1)\right)$ so that, for $a^{\prime}=a^{\prime \prime}+a^{\prime \prime \prime}$, one has $\Delta_{1,1} b^{\prime \prime}=-D_{0,2} a^{\prime}$ and $\Delta_{0,2} a^{\prime}=0$. We have proven this way 
Lemma 6.11. $\left[b^{\prime \prime}\right]+\mathcal{H}^{1,1}$ is in the image of $\kappa$ if and only if

1. $\left[\Delta_{1,1}\right]\left[b^{\prime \prime}\right]=0$ so that $\Delta_{1,1} b^{\prime \prime}=-D_{0,2} a^{\prime \prime}$,

2. the cohomology class $\left[v^{3}\right] \in H^{3}\left(\pi_{0}(\Gamma), U(1)^{\pi_{0}(M)}\right)$ of the 3-cocycle $v^{3}$ corresponding, via Eq. (6.45), to $\Delta_{0,2} a^{\prime \prime} \in H^{0}\left(\Gamma^{3} \times M, U(1)\right)$ vanishes.

Now, let us study the kernel of $\kappa$. If $\left[b^{\prime}\right] \in \mathcal{H}^{1,1}$, i.e. $b^{\prime}=\Delta_{1,0} e+D_{0,1} f^{\prime}$ for $e \in$ $A^{1}\left(\mathcal{O}^{0}\right)$ with $D_{1,0} e=0$ and $f^{\prime} \in A^{0}\left(\mathcal{O}^{1}\right)$, then $\Delta_{1,1} b^{\prime}=\Delta_{1,1} D_{0,1} f^{\prime}=D_{0,2} \Delta_{0,1} f^{\prime}$ so that

$$
a^{\prime}+\Delta_{0,1} f^{\prime} \in \operatorname{ker} D_{0,2} \cong H^{0}\left(\Gamma^{2} \times M, U(1)\right) .
$$

Since $\Delta_{0,2}\left(a^{\prime}+\Delta_{0,1} f^{\prime}\right)=0$, the cochain $a^{\prime}+\Delta_{0,1} f^{\prime}$ may be identified, by means of Eq. (6.45), with a 2-cocycle $v^{2}$ on group $\pi_{0}(\Gamma)$ with values in $U(1)^{\pi_{0}(M)}$. All 2-cocycles $v^{2}$ may be obtained this way by changing $a^{\prime}$ to $a^{\prime}+a^{\prime \prime}$ with $D_{0,2} a^{\prime \prime}=0=\Delta_{0,2} a^{\prime \prime}$. Since $f^{\prime}$ is defined modulo $f^{\prime \prime} \in \operatorname{ker} D_{0,1} \cong H^{0}(\Gamma \times M, U(1)), 2$-cocycle $v^{2}$ is defined modulo coboundaries of the 1 -cochains $u^{1}$ corresponding to $f^{\prime \prime}$ so that the cohomology class $\left[v^{2}\right] \in H^{2}\left(\pi_{0}(\Gamma), U(1)^{\pi_{0}(M)}\right)$ is well defined by the pair $\left(b^{\prime}, a^{\prime}\right)$ with $\left[b^{\prime}\right] \in \mathcal{H}^{1,1}$. The class $\left[v^{2}\right]$ vanishes if and only if $\left(b^{\prime}, a^{\prime}\right)$ is of the form (6.49). This shows

Lemma 6.12. The kernel of the map $\kappa$ of (6.53) may be identified with the cohomology group $H^{2}\left(\pi_{0}(\Gamma), U(1)^{\pi_{0}(M)}\right)$.

Let us look at some special cases. First, if $H^{1}(\Gamma, U(1))=\{0\}=H^{1}(M, U(1))$, then the homomorphism $\kappa$ vanishes and we obtain from Lemma 6.12:

Corollary 6.13. In the case when the connected components of $\Gamma$ and $M$ are simply connected, $\mathbb{H}^{2}(\mathcal{J}) \cong H^{2}\left(\pi_{0}(\Gamma), U(1)^{\pi_{0}(M)}\right)$.

This is the result that gives, e.g., the classification of $Z$-equivariant structures on gerbe $\mathcal{G}_{k}$ over $\tilde{G}$ for $Z \subset \tilde{Z}$ acting by multiplication, see [24,26,27].

Suppose now that $\Gamma$ is connected so that $\Gamma=\tilde{\Gamma} / \tilde{Z}_{\Gamma}$, where $\tilde{\Gamma}$ is a simply connected Lie group and $\tilde{Z}_{\Gamma}$ is a subgroup of the center of $\tilde{\Gamma}$. One has $H_{1}(\Gamma) \cong \pi_{1}(\Gamma) \cong \tilde{Z}_{\Gamma}$. Lemma 6.12 implies in that case that $\kappa$ is injective onto its image which, by Lemma 6.11 and Eq. (6.37), is composed of the cosets $\left[b^{\prime \prime}\right]+\mathcal{H}^{1,1}$ such that $\left[b^{\prime \prime}\right]_{2}([1])=0$ in the decomposition (6.25) and (6.26). From the explicit form (6.51) of $\mathcal{H}^{1,1}$, we then infer

Corollary 6.14. If the group $\Gamma$ and manifold $M$ are connected, then

$$
\mathbb{H}^{2}(\mathcal{J}) \cong H^{1}(\Gamma, U(1)) /\left[r_{m}^{*}\right]\left(H^{1}(M, U(1))\right) \cong Z_{M}^{*},
$$

where $Z_{M}^{*}$ is the group of characters of the kernel $Z_{M}$ of the homomorphism from $H_{1}(\Gamma)$ to $H_{1}(M)$ induced by the map $\gamma \stackrel{r_{m}}{\longmapsto} \gamma m$.

In particular, we have

Corollary 6.15. For the $\Gamma$-space $M=G$ in the coset-model context, see Definition 4.6, $Z_{M}=\tilde{Z}_{\Gamma}$ so that

$$
\mathbb{H}^{2}(\mathcal{J}) \cong H^{1}(\Gamma, U(1)) \cong \tilde{Z}_{\Gamma}^{*}
$$

and the $\Gamma$-equivariant structures on the WZW gerbes $\mathcal{G}_{k}$ over $G$ are classified by the group of characters of $H_{1}(\Gamma) \cong \pi_{1}(\Gamma) \cong \tilde{Z}_{\Gamma}$. 
Let us analyze closer the case when the $\Gamma$-space $M$ is a (left) principal $\Gamma$-bundle $\omega: M \rightarrow M^{\prime}$. By the descent Theorem 5.3, each $\Gamma$-equivariant structure on the trivial gerbe $\mathcal{I}_{0}$ relative to the vanishing 2-form descends to a flat gerbe on $M^{\prime}$ whose pullback to $M$ is 1-isomorphic to $\mathcal{I}_{0}$. Passing to isomorphism classes, one obtains the canonical injective homomorphism

$$
v: \mathbb{H}^{2}(\mathcal{J}) \rightarrow H^{2}\left(M^{\prime}, U(1)\right)
$$

that maps into the kernel of the pullback map $\left[\omega^{*}\right]: H^{2}\left(M^{\prime}, U(1)\right) \rightarrow H^{2}(M, U(1))$. Now, suppose that we are given a flat gerbe on $M^{\prime}$ whose class is in the kernel of $\left[\omega^{*}\right]$. It is easy to see, using Theorem 5.3 and Remark 5.2(2), that such a gerbe is 1-isomorphic to a gerbe that descends from the trivial gerbe $\mathcal{I}_{0}$ equipped with a $\Gamma$-equivariant structure (relative to $\rho=0$ ). This shows that $v$ maps onto the kernel of $\left[\omega^{*}\right]$. We obtain this way

Corollary 6.16. In the case when $M$ is a principal $\Gamma$-bundle, there is an exact sequence of Abelian groups

$$
0 \longrightarrow \mathbb{H}^{2}(\mathcal{J}) \stackrel{v}{\longrightarrow} H^{2}\left(M^{\prime}, U(1)\right) \stackrel{\left[\omega^{*}\right]}{\longrightarrow} H^{2}(M, U(1))
$$

that induces an isomorphism between $\mathbb{H}^{2}(\mathcal{J})$ and the kernel of $\left[\omega^{*}\right]$ in $H^{2}\left(M^{\prime}, U(1)\right)$.

If $\Gamma$ and $M$ are connected, then the exact sequence (6.58) is induced, by virtue of Corollary 6.14 , by the cohomology exact sequence for the $\Gamma$-bundle $\omega: M \rightarrow M^{\prime}$ $[10,49]$

$H^{1}(M, U(1)) \stackrel{\left[r_{m}^{*}\right]}{\longrightarrow} H^{1}(\Gamma, U(1)) \stackrel{\tau}{\longrightarrow} H^{2}\left(M^{\prime}, U(1)\right) \stackrel{\left[\omega^{*}\right]}{\longrightarrow} H^{2}(M, U(1))$.

The middle arrow $\tau$ may be easily described in terms of the classifying space $B \Gamma$ of group $\Gamma$. The transgression map $H^{2}(B \Gamma, U(1)) \rightarrow H^{1}(\Gamma, U(1))$ is an isomorphism for connected $\Gamma$. Its composition with $\tau$ is given by the pullback map from $H^{2}(B \Gamma, U(1))$ to $H^{2}\left(M^{\prime}, U(1)\right)$ along the classifying map $f_{\omega}: M^{\prime} \rightarrow B \Gamma$ for the principal bundle $\omega: M \rightarrow M^{\prime}$. In Appendix 6, we describe an equivalent construction of homomorphism $\tau$. That construction, carried out in terms of line bundles and gerbes, will be used below.

6.6. Ambiguity of gauged amplitudes. Let us recall from Sect. 5.2 how the WZ amplitudes coupled to a topologically non-trivial gauge field $\mathcal{A}$ in the principal $\Gamma$-bundle $P$ over the worldsheet $\Sigma$ were defined. They were given by the holonomy of gerbe $\mathcal{G}_{\mathcal{A}}$ over the associated bundle $P_{M}=P \times_{\Gamma} M$, see Definition 5.7. That gerbe was obtained via Theorem 5.3 from gerbe $\tilde{\mathcal{G}}_{\mathcal{A}}=\mathcal{I}_{\tilde{\rho}_{\mathcal{A}}} \otimes \mathcal{G}_{2}$ over $\tilde{M}=P \times M$ equipped with a $\Gamma$-equivariant structure (relative to $\rho=0$ ) induced from that of $\mathcal{G}$. Let us use the subscript $M$ or $\tilde{M}$ to distinguish between the two cases of $\Gamma$-spaces. If we change the isomorphism class of a $\Gamma$-equivariant structure on $\mathcal{G}$ by a class $K \in \mathbb{H}^{2}\left(\mathcal{J}_{M}\right)$, then a quick inspection of the proof of Proposition 5.5 shows that the isomorphism class of the induced $\Gamma$-equivariant structure on $\tilde{\mathcal{G}}_{\mathcal{A}}$ changes by the class $K_{2} \in \mathbb{H}^{2}\left(\mathcal{J}_{\tilde{M}}\right)$ obtained by the pullbacks along the projection $\mathrm{pr}_{2}$ of $P \times M$ on the second factor. The isomorphism class of the descended gerbe $\mathcal{G}_{\mathcal{A}}$ changes then, according to the discussion from Sect. 6.5, by

$$
v_{\tilde{M}}\left(K_{2}\right) \in H^{2}\left(P_{M}, U(1)\right) \cong H \operatorname{Hom}\left(H_{2}\left(P_{M}\right), U(1)\right) .
$$


Viewed as a character of $H_{2}\left(P_{M}\right)$, class $v_{\tilde{M}}\left(K_{2}\right)$ describes the change of the holonomy of the gerbe $\mathcal{G}_{\mathcal{A}}$. We obtain this way

Corollary 6.17. Under the change of the isomorphism class of a $\Gamma$-equivariant structure on gerbe $\mathcal{G}$ over $M$ by a class $K \in \mathbb{H}^{2}\left(\mathcal{J}_{M}\right)$, the WZ amplitude (5.23) of a section $\Phi: \Sigma \rightarrow P_{M}$ of the associated bundle is multiplied by the $U(1)$ phase $\left\langle[\Phi], v_{\tilde{M}}\left(K_{2}\right)\right\rangle$, where $[\Phi]$ denotes the homology class of $\Phi$.

Remark 6.18. The dependence of the gauged WZ amplitudes on the choice of an equivariant structure is another manifestation of the phenomenon of "discrete torsion" [53].

In the particular situation where manifolds $\Gamma, M$ and $\Sigma$ are connected, Corollary 6.14 implies that

$$
\mathbb{H}^{2}\left(\mathcal{J}_{M}\right) \cong Z_{M}^{*}, \quad \mathbb{H}^{2}\left(\mathcal{J}_{\tilde{M}}\right) \cong Z_{\tilde{M}}^{*}
$$

We shall denote by $\chi_{K}$ the character of $Z_{M}$ corresponding to $K \in \mathbb{H}^{2}\left(\mathcal{J}_{M}\right)$ and by $\chi_{\tilde{K}}$ the one of $Z_{\tilde{M}}$ corresponding to $\tilde{K} \in \mathbb{H}^{2}\left(\mathcal{J}_{\tilde{M}}\right)$. The relation $p r_{2} \circ r_{(p, m)}=r_{m}$ for $(p, m) \in \tilde{M}$ implies the inclusion $Z_{\tilde{M}} \subset Z_{M}$. The map $Z_{M}^{*} \ni \chi_{K} \mapsto \chi_{K_{2}} \in Z_{\tilde{M}}^{*}$ is now given by the restriction of the characters, whereas the homomorphism $v_{\tilde{M}}$ is induced by the map $\tau_{\tilde{M}}: H^{1}(\Gamma, U(1)) \rightarrow H^{2}\left(P_{M}, U(1)\right)$ of the exact sequence (6.59). The problem of ambiguities of the gauged WZ amplitudes may be completely settled in this case employing a construction of homomorphism $\tau_{\tilde{M}}$ along the lines of Appendix 6 and an explicit description of principal $\Gamma$-bundles over $\Sigma$ [33].

Up to isomorphism, such bundles may be obtained by gluing $D \times \Gamma$ and $(\Sigma \backslash \dot{D}) \times \Gamma$, where $D$ is a closed unit disc embedded into $\Sigma$ and $\dot{D}$ its interior, via the identification

$$
D \times \Gamma \ni\left(\mathrm{e}^{i \sigma}, \gamma\left(\mathrm{e}^{i \sigma}\right) \gamma\right)=\left(\mathrm{e}^{i \sigma}, \gamma\right) \in(\Sigma \backslash \dot{D}) \times \Gamma
$$

for a transition loop $S^{1} \ni e^{i \sigma} \mapsto \gamma\left(\mathrm{e}^{i \sigma}\right) \in \Gamma$ that we assume based at the unit element: $\gamma(1)=1$. The $\Gamma$-bundle $P$ depends, up to isomorphism, only on the element $z_{P} \in \tilde{Z}_{\Gamma} \cong \pi_{1}(\Gamma)$ corresponding to the homotopy class of the transition loop. The associated bundle $P_{M}$ is then obtained by gluing $D \times M$ and $(\Sigma \backslash \dot{D}) \times M$ via the identification

$$
D \times M \ni\left(\mathrm{e}^{i \sigma}, \gamma\left(\mathrm{e}^{i \sigma}\right) m\right)=\left(\mathrm{e}^{i \sigma}, m\right) \in(\Sigma \backslash \dot{D}) \times M
$$

A global section $\Phi: \Sigma \rightarrow P_{M}$ is given by two maps

$$
D \ni x \mapsto \phi_{1}(x) \in M \text { and }(\Sigma \backslash \dot{D}) \ni x \mapsto \phi_{2}(x) \in M
$$

such that

$$
\phi_{1}\left(\mathrm{e}^{i \sigma}\right)=\gamma\left(\mathrm{e}^{i \sigma}\right) \phi_{2}\left(\mathrm{e}^{i \sigma}\right) .
$$

According to Appendix 6, the homomorphism $\tau_{\tilde{M}}$, mapping $H^{1}(\Gamma, U(1)) \cong \tilde{Z}_{\Gamma}^{*}$ to $H^{2}\left(P_{M}, U(1)\right)$, associates to a character $\chi \in \tilde{Z}_{\Gamma}^{*}$ the 1 -isomorphism class of a flat gerbe $\mathcal{G}_{\chi}$ on $P_{M}$. Consequently, the phase $\left\langle[\Phi], v_{\tilde{M}}\left(K_{2}\right)\right\rangle$ is equal to the holonomy $\operatorname{Hol}_{\mathcal{G}_{\chi}}(\Phi)$ for a character $\chi$ of $\tilde{Z}_{\Gamma}$ extending $\chi_{K}$. The flat gerbe $\mathcal{G}_{\chi}$ may be trivialized over $D \times M$ 
and $(\Sigma \backslash \dot{D}) \times M$. It is then given by a transition line bundle [32] over $S^{1} \times M$ obtained by pulling back the flat line bundle $L_{\chi}$ over $\Gamma$, described in Appendix 6, along the map

$$
S^{1} \times M \ni\left(\mathrm{e}^{i \sigma}, m\right) \longmapsto \gamma\left(\mathrm{e}^{i \sigma}\right) \in \Gamma .
$$

Using such a presentation of gerbe $\mathcal{G}_{\chi}$, it is easy to see from the geometric definition of the holonomy of gerbes, see, e.g., [23], that the phase $\operatorname{Hol}_{\mathcal{G}_{\chi}}(\Phi)$ is given by the holonomy of the loop $\mathrm{e}^{i \sigma} \mapsto \gamma\left(\mathrm{e}^{i \sigma}\right)$ in the line bundle $L_{\chi}$ over $\Gamma$. The latter is equal to the value of the character $\chi$ on the element $z_{P} \in \tilde{Z}_{\Gamma}$.

The above phase should be independent of the extension $\chi$ of the character $\chi_{K}$ from the subgroup $Z_{M}$ to $\tilde{Z}_{\Gamma}$. This does not seem evident. Here is the resolution of the puzzle. Let $\phi_{i}$ be the maps representing section $\Phi$ of $P_{M}$. As a boundary value of a map from the disc to $M$, the 1-cycle

$$
S^{1} \ni \mathrm{e}^{i \sigma} \mapsto \phi_{2}\left(\mathrm{e}^{i \sigma}\right) \in M
$$

is homologous to a constant 1-cycle. Hence the 1-cycle

$$
S^{1} \ni \mathrm{e}^{i \sigma} \mapsto \phi_{1}\left(\mathrm{e}^{i \sigma}\right)=\gamma\left(\mathrm{e}^{i \sigma}\right) \phi_{2}\left(\mathrm{e}^{i \sigma}\right),
$$

which is a boundary value of a map from $\Sigma \backslash \dot{D}$ to $M$ and, as such, has a trivial class in $H_{1}(M)$, is homologous to

$$
S^{1} \ni \mathrm{e}^{i \sigma} \mapsto \gamma\left(\mathrm{e}^{i \sigma}\right) m
$$

for any point $m \in M$. But the triviality of the class in $H_{1}(M)$ of the latter 1-cycle is just the condition that $z_{P} \in Z_{M}$. Note that in the coset context, there always exists a section $\Phi \equiv 1$ of the associated bundle given by $\phi_{i} \equiv 1$. In that case $z_{P}$ always belongs to $Z_{M}=\tilde{Z}_{\Gamma}$, see Corollary 6.15 . We may summarize the above discussion in

Theorem 6.19. Let $\Gamma, M$, and $\Sigma$ be connected and $P$ be the principal $\Gamma$-bundle over $\Sigma$ associated to $z_{P} \in \pi_{1}(\Gamma)$. Then

1. if $z_{P} \notin Z_{M}$ then there are no global sections $\Phi$ of the associated bundle $P_{M}$;

2. for any global section $\Phi: \Sigma \rightarrow P_{M}$,

$$
\left\langle[\Phi], v_{\tilde{M}}\left(K_{2}\right)\right\rangle=\chi_{K}\left(z_{P}\right) \in U(1) .
$$

Corollary 6.20. Under the same assumptions, a change of the isomorphism class of a $\Gamma$-equivariant structure on gerbe $\mathcal{G}$ over $M$ by a class $K \in \mathbb{H}^{2}\left(\mathcal{J}_{M}\right)$ identified with a character $\chi_{K} \in Z_{M}^{*}$ multiplies the WZ amplitude (5.23) of a section $\Phi: \Sigma \rightarrow P_{M}$ by $\chi_{K}\left(z_{P}\right)$.

In particular, if $P$ is trivializable then $z_{P}=1$ and the gauged $\mathrm{WZ}$ amplitudes are independent of the choice of a $\Gamma$-equivariant structure (and may be defined in more general circumstances discussed in the first part of the paper). 
6.7. Fixed-point resolved coset partition functions. In [33], K. Hori studied an example of the coset theory based on the WZW model with simply connected group $\tilde{G}=$ $S U(2) \times S U(2)$ at level $(k, 2)$ and with gauged adjoint action of $\Gamma=\operatorname{diag}(S U(2)) /$ $\operatorname{diag}\left(\mathbf{Z}_{2}\right)$. Let $b_{\left(j_{1}, j_{2}\right), j^{\prime}}(\tau)$ denote the corresponding branching functions for spins $j_{1}=0, \frac{1}{2}, \ldots, \frac{k}{2}, j_{2}=0, \frac{1}{2}, 1$, and $j^{\prime}=0, \frac{1}{2}, \ldots, \frac{k}{2}+1$. As a consequence of Eqs. (4.56) and (4.57), functions $b_{\left(j_{1}, j_{2}\right), j^{\prime}}(\tau)$ vanish if $j_{1}+j_{2}-j^{\prime}$ is not an integer and are unchanged by the joint spectral flow

$$
\left(j_{1}, j_{2}, j^{\prime}\right) \longmapsto\left(\frac{k}{2}-j_{1}, 1-j_{2}, \frac{k+2}{2}-j^{\prime}\right) .
$$

It follows from Eq. (4.60) for $G^{\prime}=\tilde{G}$ that the contribution to the coset partition function of the sector with topologically trivial gauge fields is equal to

$$
\mathcal{Z}_{\text {triv }}=\sum_{\left[\left(j_{1}, j_{2}\right), j^{\prime}\right]} \frac{1}{\left|S_{\left[\left(j_{1}, j_{2}\right), j^{\prime}\right]}\right|}\left|b_{\left(j_{1}, j_{2}\right), j}(\tau)\right|^{2},
$$

where $\left[\left(j_{1}, j_{2}\right), j^{\prime}\right]$ runs through the orbits of the spectral flow $(6.71)$ and $S_{\left[\left(j_{1}, j_{2}\right), j^{\prime}\right]}$ denotes the corresponding stabilizer subgroups of $\tilde{Z}_{\Gamma} \cdot\left|S_{\left[\left(j_{1}, j_{2}\right), j^{\prime}\right]}\right|=1$ for the twopoint orbits and $\left|S_{\left[\left(j_{1}, j_{2}\right), j^{\prime}\right]}\right|=2$ for the one-point ones composed of fixed points of the spectral flow. For $k$ odd, one always has $\left|S_{\left[\left(j_{1}, j_{2}\right), j^{\prime}\right]}\right|=1$. In that case, the contribution $\mathcal{Z}_{\text {ntriv }}(\tau)$ of the sector with topologically non-trivial gauge fields to the partition function vanishes [33]. For $k$ even, however, there is one fixed point orbit $\left[\left(\frac{k}{4}, \frac{1}{2}\right), \frac{k+2}{4}\right]$ with $\left|S_{\left[\left(j_{1}, j_{2}\right), j^{\prime}\right]}\right|=2$. Using the supersymmetry present in the above coset model, Hori showed the decomposition

$$
b_{\left(\frac{k}{4}, \frac{1}{2}\right), \frac{k+2}{4}}(\tau)=b^{+}(\tau)+b^{-}(\tau) \quad \text { with } \quad b^{+}(\tau)-b^{-}(\tau)=1,
$$

where $b^{ \pm}(\tau)$ collects the contribution to $b_{\left(\frac{k}{4}, \frac{1}{2}\right), \frac{k+2}{4}}(\tau)$ of states with $(-1)^{F}= \pm 1$ for $F$ the fermion number. In the terminology of [16], decomposition (6.73) gives the resolution of the fixed-point branching function. Further analysis in [33] established that $\mathcal{Z}_{\text {ntriv }}(\tau)$ is $\tau$-independent and postulated the equality $Z_{\text {ntriv }}=\frac{1}{2}$. In that case, the sum of the fixed point contribution to $\mathcal{Z}_{\text {triv }}(\tau)$ and of $\mathcal{Z}_{\text {ntriv }}$ gives

$$
\frac{1}{2}\left|b^{+}(\tau)+b^{-}(\tau)\right|^{2}+\frac{1}{2}=\left|b^{+}(\tau)\right|^{2}+\left|b^{-}(\tau)\right|^{2},
$$

which is the diagonal sum of the resolved fixed-point branching functions, as proposed in [16]. On the other hand, Hori argued that a different $\theta$-vacuum of the coset theory should lead to $\mathcal{Z}_{\text {ntriv }}=-\frac{1}{2}$. In the latter case, one obtains

$$
\frac{1}{2}\left|b^{+}(\tau)+b^{-}(\tau)\right|^{2}-\frac{1}{2}=b^{+}(\tau) \overline{b^{-}(\tau)}+b^{-}(\tau) \overline{b^{+}(\tau)},
$$

i.e. a non-diagonal combination of the resolved fixed-point branching functions. The latter choice was not discussed in [16]. Since $\tilde{Z}_{\Gamma}=\mathbf{Z}_{\mathbf{2}}=Z_{M}$ in the present case, it follows from Corollary 6.20 that the sign ambiguity of $Z_{n t r i v}$ is due to the freedom of choice of an $S O(3)$-equivariant structure on gerbe $\mathcal{G}_{(k, 2)}$ over $S U(2) \times S U(2)$.

Based on the analysis of [16], one may generalize the above discussion and conjecture explicit expressions for the contributions $\mathcal{Z}_{P}^{G^{\prime} / \Gamma}(\tau)$ to the coset partition functions of gauge fields in principal $\Gamma$-bundles $P$ for groups $G^{\prime}=\tilde{G} / Z^{\prime}$ where $Z^{\prime} \subset \tilde{Z}$ is a 
non-anomalous subgroup, see Sect. 4.4. Let $b_{k, \Lambda, \lambda}^{\hat{\mathbf{g}}, \hat{\mathbf{h}}, \tilde{z}}(\tau)$ be the so-called twining branching functions introduced in [16] for $\tilde{z} \in \tilde{Z}_{\Gamma}$ with spectral flow fixing $(\Lambda, \lambda)$ and set to zero otherwise. The formula

$$
\mathcal{Z}_{P}^{G^{\prime} / \Gamma}(\tau)=\sum_{z \in Z^{\prime}} \sum_{[\Lambda, \lambda] \in \mathcal{P}_{z}} \frac{1}{\left|S_{[\Lambda, \lambda]}\right|} b_{k, z^{-1} \Lambda, \lambda}^{\hat{\mathbf{g}}, \hat{\mathbf{h}}, z P}(\tau) \overline{b_{k, \Lambda, \lambda}^{\hat{\mathbf{g}}, \hat{\mathbf{h}}, z_{P}}(\tau)}
$$

should hold for a special choice of the $\Gamma$-equivariant structure on the gerbe $\mathcal{G}_{k}$ over $G^{\prime}$ (for $G^{\prime}=\tilde{G}$, see Remark 7.2 below). Since for $z_{P}=1$ the twining branching functions coincide with the standard ones, the above expression gives correctly the contribution of the sector with topologically trivial gauge fields, see Eq. (4.60). Summing over the isomorphism classes of $\Gamma$-principal bundles, i.e. over $z_{P} \in \tilde{Z}_{\Gamma}$, one obtains, with the use of the Plancherel formula for the isotropy groups $S_{[\Lambda, \lambda]} \subset \tilde{Z}_{\Gamma}$, the expression for the total partition function

$$
\mathcal{Z}_{\text {tot }}^{G^{\prime} / \Gamma}(\tau)=\sum_{z \in Z^{\prime}} \sum_{[\Lambda, \lambda] \in \mathcal{P}_{z}} \sum_{\chi \in S_{[\Lambda, \lambda]}^{*}} b_{k, z^{-1} \Lambda, \lambda}^{\hat{\mathbf{g}}, \hat{\mathbf{h}}, \chi}(\tau) \overline{b_{k, \Lambda, \lambda}^{\hat{\mathbf{g}}, \hat{\mathbf{h}}, \chi}(\tau)}
$$

in terms of the resolved fixed-point branching functions [16]

$$
b_{k, \Lambda, \lambda}^{\hat{\mathbf{g}}, \hat{\mathbf{h}}, \chi}(\tau)=\frac{1}{\left|S_{[\Lambda, \lambda]}\right|} \sum_{\tilde{z} \in S_{[\Lambda, \lambda]}} \chi(\tilde{z})^{-1} b_{k, \Lambda, \lambda}^{\hat{\mathbf{g}}, \hat{\mathbf{h}}, \tilde{z}}(\tau) .
$$

satisfying the sum rule

$$
\sum_{\chi \in S_{[\Lambda, \lambda]}^{*}} b_{k, \Lambda, \lambda}^{\hat{\mathbf{g}}, \hat{\mathbf{h}}, \chi}(\tau)=b_{k, \Lambda, \lambda}^{\hat{\mathbf{g}}, \hat{\mathbf{h}}}(\tau)
$$

On the other hand, the twist of the $\Gamma$-equivariant structure by a character $\chi_{K} \in \tilde{Z}_{\Gamma}^{*}$ introduces the factor $\chi_{K}\left(z_{P}\right)$ on the right hand side of Eq. (6.76) giving rise to the modified total partition function

$$
\mathcal{Z}_{\text {tot }}^{G^{\prime} / \Gamma}(\tau)=\sum_{z \in Z^{\prime}} \sum_{[\Lambda, \lambda] \in \mathcal{P}_{z}} \sum_{\chi \in S_{[\Lambda, \lambda]}^{*}} b_{k, z^{-1} \Lambda, \lambda}^{\hat{\mathbf{g}}, \hat{\mathbf{h}}, \chi}(\tau) \overline{b_{k, \Lambda, \lambda}^{\hat{\mathbf{g}}, \hat{\mathbf{h}}, \chi_{K} \chi}(\tau)}
$$

For $G^{\prime}=\tilde{G}$, Eq. (6.77) gives the coset partition function in terms of the sum of squares of the fixed-point resolved branching functions, as postulated in [16].

\section{Ad-Equivariant WZW Gerbes Over Simply Connected Groups}

In order to illustrate the concept of $\Gamma$-equivariant gerbes, we shall return to the situation discussed in Sect. 4.2 involving the WZW gerbes $\mathcal{G}_{k}$ over connected compact simple groups $G=\tilde{G} / Z$ viewed as $\Gamma$-spaces for $\Gamma=\tilde{G} / \tilde{Z}$ acting by the adjoint action. Recall that Theorem 6.9 states that gerbes $\mathcal{G}_{k}$ possess $\Gamma$-equivariant structures whenever the phases (4.22) are trivial, so always for $G=\tilde{G}$. Such structures are composed of 1-isomorphism $\alpha$ and 2-isomorphism $\beta$, see Definition 5.1. They are classified by the dual 
group of $\tilde{Z}$, see Corollary 6.15. What follows is devoted to an explicit construction of $\Gamma$-equivariant structures on gerbes $\mathcal{G}_{k}$ over simply connected groups $\tilde{G}$.

Instead of the local data formalism used in Sect. 6, we shall employ below a geometric presentation of gerbes and their 1- and 2-isomorphisms, see, e.g., [27]. In such a presentation, a gerbe $\mathcal{G}$ over $M$ with curvature $H$ is a quadruple $(Y, B, L, \mu)$, where $\pi: Y \rightarrow M$ is a surjective submersion, $B$ is a 2 -form on $Y$, called curving, such that $d B=\pi^{*} H, L$ is a line bundle over the fiber-product $Y^{[2]}=Y \times_{M} Y$ with curvature $F=B_{2}-B_{1}$, and $\mu: L_{12} \otimes L_{23} \rightarrow L_{13}$ is an isomorphism of line bundles over $Y^{[3]}=Y \times_{M} Y \times_{M} Y$ that defines a groupoid structure on $L \rightrightarrows Y$ (the subscripts denote here the pullbacks along projections from $Y^{[p]} \rightarrow Y^{[q]}$ ). An explicit geometric construction of gerbes $\mathcal{G}_{k}$ over $M=\tilde{G}$ with $k \in \mathbb{Z}$ was given in [42] and is somewhat involved. We shall use here its description from [24], see also Sec. 4.1 of [20].

7.1. WZW gerbes over compact simply connected simple Lie groups. As before, coroots, coweights, roots and coroots will be considered as elements of the imaginary Cartan subalgebra $i \mathbf{t} \subset$ ig identified with its dual with the help of the bilinear form tr. The normalization of tr makes the length squared of long roots equal to 2. $\alpha_{i}, \alpha_{i}^{\vee}, \lambda_{i}$ and $\lambda_{i}^{\vee}$, where $i=1, \ldots, r$, will denote the simple roots, coroots, weights and coweights, respectively, with $r$ the rank of $\mathbf{g}$. The highest root $\phi=\sum_{i} k_{i} \alpha_{i}$, where the positive integers $k_{i}$ are the Kac labels. Denote by $\mathcal{A}_{W} \subset i$ t the positive Weyl alcove. $\mathcal{A}_{W}$ is a simplex with vertices $\tau_{i}=\frac{1}{k_{i}} \lambda_{i}^{\vee}, i=1, \ldots, r$, and $\tau_{0}=0$. For $i \in R:=\{0,1, \ldots, r\}$, let

$$
\begin{aligned}
& \mathcal{A}_{i}=\left\{\tau \in \mathcal{A}_{W} \mid \tau=\sum_{j} s_{j} \tau_{j} \text { with } s_{i}>0\right\}, \\
& O_{i}=\left\{g=A d_{h_{g}}\left(\mathrm{e}^{2 \pi i \tau}\right) \mid h_{g} \in \tilde{G}, \tau \in \mathcal{A}_{i}\right\},
\end{aligned}
$$

and, for $I \subset R$, let $\mathcal{A}_{I}=\cap_{i \in I} \mathcal{A}_{i}$ and $O_{I}=\cap_{i \in I} O_{i}$. Subsets $O_{I}$ of $\tilde{G}$ are open and $A d$-invariant. They are composed of elements $g=A d_{h_{g}}\left(\mathrm{e}^{2 \pi i \tau}\right)$ with $h_{g} \in G$ and $\tau \in \mathcal{A}_{I}$. The expressions

$$
B_{i}=\frac{k}{4 \pi} \operatorname{tr}\left(\Theta\left(h_{g}\right) A d_{\mathrm{e} 2 \pi i \tau}\left(\Theta\left(h_{g}\right)\right)+2 \pi i\left(\tau-\tau_{i}\right)\left[\Theta\left(h_{g}\right), \Theta\left(h_{g}\right)\right]\right),
$$

where $\Theta\left(h_{g}\right)=h_{g}^{-1} d h_{g}$, define smooth 2-forms on $O_{i}$ such that $d B_{i}=H_{k} \mid O_{i}$. For groups $S U(n)$, it is enough to take $Y=\sqcup_{i} O_{i}$, see [7,23]. In order to have a unique construction of gerbes $\mathcal{G}_{k}$ for all compact simply connected simple Lie groups, one makes a more involved choice [42].

Consider the stabilizer subgroups,

$$
G_{I}=\left\{\gamma \in \tilde{G} \mid \gamma \mathrm{e}^{2 \pi i \tau} \gamma^{-1}=\mathrm{e}^{2 \pi i \tau} \text { for (any) } \tau \in \mathcal{A}_{I} \backslash \cup_{i \notin I} \mathcal{A}_{i}\right\}
$$

In particular, $G_{i}$ is composed of the elements of $\tilde{G}$ that commute with $\mathrm{e}^{2 \pi i \tau_{i}}$. The Cartan subgroup $T \subset \tilde{G}$ is contained in all $G_{I}$. The maps

$$
\mathcal{O}_{I} \ni g=A d_{h_{g}}\left(\mathrm{e}^{2 \pi i \tau}\right) \stackrel{\eta_{I}}{\longmapsto} \quad h_{g} G_{I} \in G / G_{I}
$$


are well-defined because the adjoint-action stabilizers of $\mathrm{e}^{2 \pi i \tau}$ for $\tau \in \mathcal{A}_{I}$ are contained in $G_{I}$. They are smooth, see Sec. 5.1 of [42]. One introduces principal $G_{I}$-bundles $\pi_{I}: P_{I} \rightarrow O_{I}$

$$
P_{I}=\left\{(g, h) \in O_{I} \times \tilde{G} \mid \eta_{I}(g)=h G_{I}\right\} .
$$

For the gerbes $\mathcal{G}_{k}=(Y, B, L, \mu)$, one sets

$$
Y=\underset{i \in R}{\sqcup} P_{i}
$$

with $\pi: Y \rightarrow \tilde{G}$ restricting to $\pi_{i}$ on $P_{i}$ and the 2 -form $B$ restricting to $\pi_{i}^{*} B_{i}$. Let

$$
\hat{Y}_{i_{1} \ldots i_{n}}=P_{I} \times G_{i_{1}} \times \cdots \times G_{i_{n}} \text { and } Y_{i_{1} \ldots i_{n}}=\hat{Y}_{i_{1} \ldots i_{n}} / G_{I}
$$

for $I=\left\{i_{1}, \ldots, i_{n}\right\}$, and for $G_{I}$ acting on $\hat{Y}_{i_{1} \ldots i_{n}}$ diagonally by the right multiplication. The fiber power $Y^{[n]}$ of $Y$ may be identified with the disjoint union of $Y_{i_{1} \ldots i_{n}}$ by assigning to the $G_{I^{-}}$-orbit of $\left((g, h), \gamma_{i_{1}}, \ldots, \gamma_{i_{n}}\right)$ the $n$-tuple $\left(y_{1}, \ldots, y_{n}\right) \in Y^{[n]}$ with $y_{m}=\left(g, h \gamma_{i_{m}}^{-1}\right)$,

$$
Y^{[n]} \cong \underset{\left(i_{1}, \ldots, i_{n}\right)}{\sqcup} Y_{i_{1} \ldots i_{n}}
$$

The construction of the line bundle $L$ over $Y^{[2]}$ uses more detailed properties of the stabilizer groups $G_{I}$. For $I \subset J \subset R, G_{J}$ is contained in $G_{I}$. The smallest of those groups, $G_{R}$, coincides with the Cartan subgroup $T$ of $\tilde{G}$. Groups $G_{I}$ are connected but not necessarily simply connected. Let $\mathbf{g}_{I} \supset \mathbf{t}$ denote the Lie algebra of $G_{I}$, and let $\mathrm{e}_{I}$ be the exponential map from $\mathbf{g}_{I}$ to the universal cover $\tilde{G}_{I}$. One has

$$
G_{I}=\tilde{G}_{I} / Z_{I} \text { for } Z_{I}=\mathrm{e}_{I}^{2 \pi i Q^{\vee}},
$$

where $Q^{\vee} \subset \mathbf{t}$ is the coroot lattice of $\mathbf{g}$. The exponential map e I $_{I}$ maps $\mathbf{t}$ to the Abelian subgroup $\tilde{T}_{I} \subset \tilde{G}_{I}$. For $I \subset J$, the group $\tilde{G}_{J}$ maps naturally into $\tilde{G}_{I}$ and $Z_{J}$ into $Z_{I}$. One shows that the formula

$$
\chi_{i}\left(\mathrm{e}_{i}^{2 \pi i \tau}\right)=\mathrm{e}^{2 \pi i t r \tau_{i} \tau}
$$

for $\tau \in \mathbf{t}$ defines a character $\chi_{i}: \tilde{T}_{i} \rightarrow U(1)$. By restriction, $\chi_{i}$ determines a character of $Z_{i}$. One may also define a 1 -dimensional representation $\chi_{i j}: \tilde{G}_{i j} \rightarrow U(1)$ by the formula

$$
\chi_{i j}\left(\tilde{\gamma}_{i j}\right)=\exp \left[\frac{1}{i} \int_{\tilde{\gamma}_{i j}} a_{i j}\right],
$$

where $a_{i j}=i \operatorname{tr}\left(\tau_{j}-\tau_{i}\right) \Theta\left(\gamma_{i j}\right)$ is a closed 1-form on $G_{i j}\left(\tilde{\gamma}_{i j}\right.$ is identified with a homotopy class of a path in $G_{i j}$ starting at 1$)$. For $\tau \in i$ t one has:

$$
\chi_{i j}\left(\mathrm{e}_{i j}^{2 \pi i \tau}\right)=\chi_{i}\left(\mathrm{e}_{i}^{2 \pi i \tau}\right)^{-1} \chi_{j}\left(\mathrm{e}_{j}^{2 \pi i \tau}\right) .
$$

Besides $\chi_{i j}\left(\tilde{\gamma}_{i j}\right)=\chi_{j i}\left(\tilde{\gamma}_{i j}\right)^{-1}$, and for $\tilde{\gamma}_{i j k} \in \tilde{G}_{i j k}$,

$$
\chi_{i j}\left(\tilde{\gamma}_{i j k}\right) \chi_{j k}\left(\tilde{\gamma}_{i j k}\right)=\chi_{i k}\left(\tilde{\gamma}_{i j k}\right) .
$$


Over space $\hat{Y}_{i j}$, there is a line bundle $\hat{L}_{i j}$ whose fiber over $\left((g, h), \gamma_{i}, \gamma_{j}\right)$ is composed of the equivalence classes $\left[\tilde{\gamma}_{i}, \tilde{\gamma}_{j}, u_{i j}\right]_{i j}$ with respect to the relation

$$
\left(\tilde{\gamma}_{i}, \tilde{\gamma}_{j}, u_{i j}\right) \underset{i j}{\sim}\left(\tilde{\gamma}_{i} \zeta_{i}, \tilde{\gamma}_{j} \zeta_{j}, \chi_{i}\left(\zeta_{i}\right)^{k} \chi_{j}\left(\zeta_{j}\right)^{-k} u_{i j}\right)
$$

for $\tilde{\gamma}_{i} \in \tilde{G}_{i}, \tilde{\gamma}_{j} \in \tilde{G}_{j}$ projecting to $\gamma_{i} \in G_{i}$ and $\gamma_{j} \in G_{j}$, respectively, and $u_{i j} \in$ C, $\zeta_{i} \in Z_{i}, \zeta_{j} \in Z_{j}$. One twists the natural flat structure of $\hat{L}_{i j}$ by the connection form

$$
\hat{A}_{i j}=i k \operatorname{tr}\left(\tau_{j}-\tau_{i}\right) \Theta(h)
$$

The right action of $G_{i j}$ on $\hat{Y}_{i j}$ lifts to the action on $\hat{L}_{i j}$ defined by

$$
\left((g, h),\left[\tilde{\gamma}_{i}, \tilde{\gamma}_{j}, u_{i j}\right]_{i j}\right) \longmapsto\left((g, h \gamma),\left[\tilde{\gamma}_{i} \tilde{\gamma}_{i j}, \tilde{\gamma}_{j} \tilde{\gamma}_{i j}, \chi_{i j}\left(\tilde{\gamma}_{i j}\right)^{-k} u_{i j}\right]_{i j}\right)
$$

for $\gamma_{i j} \in G_{i j}$ and $\tilde{\gamma}_{i j}$ its lift to $\tilde{G}_{i j}$. The hermitian structure and the connection of $\hat{L}_{i j}$ descend to the quotient bundle $\hat{L}_{i j} / G_{i j}=L_{i j}$ over $Y_{i j}$ and the line bundle $L$ over $Y^{[2]}$ for the gerbe $\mathcal{G}_{k}$ is taken as equal to $L_{i j}$ when restricted to $Y_{i j}$. The curvature 2-form $F_{i j}$ of $L_{i j}$ lifts to $\hat{Y}_{i j}$ to the 2-form $d \hat{A}_{i j}$ that coincides with the lift to $\hat{Y}_{i j}$ of the 2-form $B_{j}-B_{i}$. This gives the required relation $F=B_{2}-B_{1}$ between the curvature $F$ of the line bundle $L$ over $Y^{[2]}$ and the curving $B$ on $Y$.

The groupoid multiplication $\mu$ of $\mathcal{G}$ is defined as follows. Let $\left((g, h), \gamma_{i}, \gamma_{j}, \gamma_{k}\right) \in$ $\hat{Y}_{i j k}$ represent $\left(y, y^{\prime}, y^{\prime \prime}\right) \in Y^{[3]}$ with $y=\left(g, h \gamma_{i}^{-1}\right), y^{\prime}=\left(g, h \gamma_{j}^{-1}\right)$ and $y^{\prime \prime}=$ $\left(g, h \gamma_{k}^{-1}\right)$ and let

$$
\ell_{i j} \in L_{\left(y, y^{\prime}\right)}, \quad \ell_{j k} \in L_{\left(y^{\prime}, y^{\prime \prime}\right)}, \quad \ell_{i k} \in L_{\left(y, y^{\prime \prime}\right)}
$$

be the elements in the appropriate fibers of $L$ given by the $G_{i j k}$-orbits of

$$
\begin{aligned}
& \hat{\ell}_{i j}=\left((g, h),\left[\tilde{\gamma}_{i}, \tilde{\gamma}_{j}, u_{i j}\right]_{i j}\right), \quad \hat{\ell}_{j k}=\left((g, h),\left[\tilde{\gamma}_{j}, \tilde{\gamma}_{k}, u_{j k}\right]_{j k}\right), \\
& \hat{\ell}_{i k}=\left((g, h),\left[\tilde{\gamma}_{i}, \tilde{\gamma}_{k}, u_{i k}\right]_{i j}\right)
\end{aligned}
$$

with $u_{i k}=u_{i j} u_{j k}$. Then

$$
\mu\left(\ell_{i j} \otimes \ell_{j k}\right)=\ell_{i k}
$$

This ends the description of gerbes $\mathcal{G}_{k}=(Y, B, L, \mu)$ over simply connected groups $\tilde{G}$.

7.2. Construction of 1-isomorphism $\alpha$. We need to compare the pullbacks of gerbe $\mathcal{G}_{k}$ to the product space $\Gamma \times \tilde{G}$. Consider first the pullback $\left(\mathcal{G}_{k}\right)_{12}$ along the adjoint action $\ell: \Gamma \times \tilde{G} \rightarrow \tilde{G}$ of $\Gamma=\tilde{G} / \tilde{Z}$ on $\tilde{G}$. One has:

$$
\left(\mathcal{G}_{k}\right)_{12}=\left(Y_{12}, B_{12}, L_{12}, \mu_{12}\right) .
$$

The adjoint action of $\tilde{G}$ on itself may be lifted to $Y$ by the map

$$
\tilde{G} \times Y \ni(\tilde{\gamma}, y) \longmapsto A d_{\tilde{\gamma}}(y) \in Y,
$$


where for $y=(g, h) \in P_{i} \subset Y, A d_{\tilde{\gamma}}(y):=\left(A d_{\tilde{\gamma}}(g), \tilde{\gamma} h\right) \in P_{i}$. The map (7.21) is constant on orbits of the action

$$
(\tilde{\gamma}, y) \mapsto\left(z \tilde{\gamma}, y z^{-1}\right)
$$

of $\tilde{Z}$ on $\tilde{G} \times Y$, where $y z^{-1}:=\left(g, h z^{-1}\right)$ for $y=(g, h) \in P_{i}$. It allows the canonical identification

$$
Y_{12} \equiv(\tilde{G} \times Y) / \tilde{Z}
$$

In this identification, the surjective submersion $\pi_{12}:(Y)_{12} \rightarrow \Gamma \times \tilde{G}$ is generated by the map $(\tilde{\gamma}, y) \mapsto(\gamma, \pi(y))$, where $\gamma \in \Gamma=\tilde{G} / \tilde{Z}$ is the canonical projection of $\tilde{\gamma}$. Similarly,

$$
Y_{12}^{[n]} \cong\left(\tilde{G} \times Y^{[n]}\right) / \tilde{Z} .
$$

The action of $\tilde{Z}$ on $\tilde{G} \times Y^{[2]}$ induced by (7.22) may be lifted to the one on $\tilde{G} \times L$ given by

$$
\left(\tilde{\gamma}, \ell_{i j}\right) \longmapsto\left(z \tilde{\gamma}, \ell_{i j} \star z^{-1}\right),
$$

where for $\ell_{i j}$ given by the $G_{i j}$-orbit (7.16) of $\hat{\ell}_{i j}=\left((g, h),\left[\tilde{\gamma}_{i}, \tilde{\gamma}_{j}, u_{i j}\right]_{i j}\right)$, the element $\ell_{i j} \star z^{-1}$ is defined as the $G_{i j}$-orbit of

$$
\hat{\ell}_{i j} \star z^{-1}:=\left((g, h),\left[\tilde{\gamma}_{i} \tilde{z}, \tilde{\gamma}_{j} \tilde{z}, \chi_{i j}(\tilde{z})^{-k} u_{i j}\right]_{i j}\right),
$$

with $\tilde{z}$ standing for any lift of $z \in \tilde{Z}$ to $\tilde{G}_{i j}$. We introduce a special symbol for this action to distinguish it from another one that will be defined below. As line bundles,

$$
L_{12} \cong(\tilde{G} \times L) / \tilde{Z} .
$$

In order to obtain the correct connection on $L_{12}$, the one on $\tilde{G} \times L$ has to be modified by twisting the flat structure on $\tilde{G} \times \hat{L}_{i j}$ by the connection 1 -form

$$
\tilde{\gamma}^{*} \hat{A}_{i j}=i k \operatorname{tr}\left(\tau_{j}-\tau_{i}\right) \Theta(\tilde{\gamma} h)
$$

rather than by $\hat{A}_{i j}$ of Eq. (7.15).

1-isomorphism $\alpha$ will compare gerbe $\left(\mathcal{G}_{k}\right)_{12}$ to $\mathcal{I}_{\rho_{k}} \otimes\left(\mathcal{G}_{k}\right)_{2}=\left(Y_{2}, B_{2}+\pi_{2}^{*} \rho_{k}, L_{2}\right.$, $\left.\mu_{2}\right)$, where $\left(\mathcal{G}_{k}\right)_{2}$ is the pullback of $\mathcal{G}_{k}$ to $\Gamma \times \tilde{G}$ along the projection to the second factor. It will be convenient to identify

$$
Y_{2}=\Gamma \times Y \cong(\tilde{G} \times Y) / \tilde{Z}
$$

where now $\tilde{Z}$ acts only on $\tilde{G}$. The projection $\pi_{2}: Y_{2} \rightarrow \Gamma \times \tilde{G}$ is induced upon this identification by the map $(\tilde{\gamma}, y) \mapsto(\gamma, \pi(y))$. Similarly,

$$
Y_{2}^{[n]}=\Gamma \times Y^{[n]} \cong\left(\tilde{G} \times Y^{[n]}\right) / \tilde{Z}, \quad L_{2}=\Gamma \times L \cong(\tilde{G} \times L) / \tilde{Z},
$$

with $\tilde{Z}$ always acting trivially on the $2^{\text {nd }}$ factor.

The first part of data for 1-isomorphism $\alpha$ is a line bundle $E$ over $W_{12}:=Y_{12} \times_{(\Gamma \times \tilde{G})}$ $Y_{2}$, see [26]. $E$ has to be equipped with a connection whose curvature form $F^{E}$ is equal 
to $\left(B_{2}+\pi_{2}^{*} \rho_{k}\right)_{2}-\left(B_{12}\right)_{1}$, where the outside subscript 1 (resp. 2$)$ refers to the pullback along the projection from $W_{12}$ to $Y_{12}$ (resp. to $Y_{2}$ ). In view of identifications (7.23) and (7.29), we obtain for the fiber-product space $W_{12}$,

$$
W_{12} \cong\left(\tilde{G} \times Y^{[2]}\right) / \tilde{Z}
$$

for the action $\left(\tilde{\gamma},\left(y, y^{\prime}\right)\right) \mapsto\left(z \tilde{\gamma},\left(y z^{-1}, y^{\prime}\right)\right)$ of $\tilde{Z}$. The projection to $Y_{12}$ is induced by the map $\left(\tilde{\gamma},\left(y, y^{\prime}\right)\right) \mapsto(\tilde{\gamma}, y)$, the one to $Y_{2}$ by $\left(\tilde{\gamma},\left(y, y^{\prime}\right)\right) \mapsto\left(\tilde{\gamma}, y^{\prime}\right)$. The composed projection $\varpi: W_{12} \rightarrow \Gamma \times \tilde{G}$ is $\left(\tilde{\gamma},\left(y, y^{\prime}\right)\right) \mapsto\left(\gamma, \pi(y)=\pi\left(y^{\prime}\right)\right)$. Line bundle $E$ over $W_{12}$ will be defined by

$$
E:=(\tilde{G} \times L) / \tilde{Z}
$$

for the action of $\tilde{Z}$,

$$
\left(\tilde{\gamma}, \ell_{i j}\right) \longmapsto\left(z \tilde{\gamma}, \ell_{i j} \cdot z^{-1}\right),
$$

where the element $\ell_{i j} \cdot z^{-1}$ defined as the $G_{i j}$-orbit of

$$
\hat{\ell}_{i j} \cdot z^{-1}:=\left((g, h),\left[\tilde{\gamma}_{i} \tilde{z}, \tilde{\gamma}_{j}, \chi_{i}(\tilde{z})^{k} \chi(z) u_{i j}\right]_{i j}\right)
$$

with $\chi: \tilde{Z} \rightarrow U(1)$ a fixed character. Note the difference between elements $\hat{\ell}_{i j} \cdot z^{-1}$ and $\hat{\ell}_{i j} \star z^{-1}$, with the latter one defined by Eq. (7.26).

The connection in line bundle $E$ requires a careful definition in order to assure that it has the desired curvature. Note that the 2 -form $\left(B_{2}+\pi_{2}^{*} \rho_{k}\right)_{2}-\left(B_{12}\right)_{1}$ on $\left(\tilde{G} \times Y_{i j}\right) / \tilde{Z} \subset$ $W_{12}$ is equal to the pullback by $\varpi$ of the 2 -form $\left(B_{j}\right)_{2}+\rho_{k}-\left(B_{i}\right)_{12}$ on $\Gamma \times O_{i j} \subset \Gamma \times \tilde{G}$. A short calculation shows that for $\gamma \in \Gamma$ and $g=A d_{h_{g}}\left(\mathrm{e}^{2 \pi i \tau}\right) \in O_{i j}$,

$$
\begin{aligned}
& \left(\left(B_{j}\right)_{2}+\rho_{k}-\left(B_{i}\right)_{12}\right)(\gamma, g) \\
& \quad=d\left(i k \operatorname{tr} A d_{h_{g}}\left(\tau-\tau_{i}\right) \Theta(\gamma)\right)+\frac{1}{2} i k \operatorname{tr}\left(\tau_{i}-\tau_{j}\right)\left[\Theta\left(h_{g}\right), \Theta\left(h_{g}\right)\right] .
\end{aligned}
$$

It was shown in [42] that the map

$$
O_{i} \ni g=A d_{h_{g}}\left(\mathrm{e}^{2 \pi i \tau}\right) \longmapsto A d_{h_{g}}\left(\tau-\tau_{i}\right) \in i \mathbf{g},
$$

denoted $\Psi_{i}$ there, is well defined and smooth so that the 1 -form $A_{i}=i k \operatorname{tr} A d_{h_{g}}\left(\tau-\tau_{i}\right)$ $\Theta(\gamma)$ is well defined and smooth on $\Gamma \times O_{i}$. On the other hand, the 2-form $B_{j}-B_{i}=$ $\frac{1}{2} i k \operatorname{tr}\left(\tau_{i}-\tau_{j}\right)\left[\Theta\left(h_{g}\right), \Theta\left(h_{g}\right)\right]$ is a well defined closed 2-form on $O_{i j}$ which, when pulled back to $Y_{i j} \subset Y^{[2]}$, coincides with the curvature form of $\left.L\right|_{Y_{i j}}=L_{i j}$. In order to assure the correct curvature of $E$, we shall additionally twist the connection of $\tilde{G} \times\left. L\right|_{Y_{i j}}$ in (7.32) by the pullbacks to $\tilde{G} \times Y_{i j}$ of the forms

$$
\hat{A}_{i}=i k \operatorname{tr} A d_{h_{g}}\left(\tau-\tau_{i}\right) \Theta(\tilde{\gamma})
$$

on $\tilde{G} \times O_{i j}$. A straightforward check shows that the resulting connection in $\tilde{G} \times L$ descends to the quotient by the action (7.33) of $\tilde{Z}$. Note that the resulting bundles $E$ differ for different characters $\chi$ of $\tilde{Z}$ by tensor factors that are pullbacks to $W_{12}$ of flat bundles over $\Gamma$. 
1-isomorphism $\alpha:\left(\mathcal{G}_{k}\right)_{12} \rightarrow \mathcal{I}_{\rho_{k}} \otimes\left(\mathcal{G}_{k}\right)_{2}$ of Definition 5.1 is an isomorphism of line bundles over $W_{12}^{[2]}$

$$
\alpha: L_{12} \otimes E_{2} \longrightarrow E_{1} \otimes L_{2}
$$

where natural pullbacks of bundles $L_{12}$ and $L_{2}$ are understood. Recalling realization (7.31) of $W_{12}$, we have

$$
W_{12}^{[2]} \cong\left(\tilde{G} \times Y^{[4]}\right) / \tilde{Z}
$$

with the action

$$
\left(\tilde{\gamma}, y_{1}, y_{1}^{\prime}, y_{2}, y_{2}^{\prime}\right) \longmapsto\left(z \tilde{\gamma},\left(y_{1} z^{-1}, y_{1}^{\prime}, y_{2} z^{-1}, y_{2}^{\prime}\right)\right)
$$

of $\tilde{Z}$. Suppose that $\left(y_{1}, y_{1}^{\prime}, y_{2}, y_{2}^{\prime}\right) \in Y_{i_{1} j_{1} i_{2} j_{2}}$ and that

$$
\ell_{i_{1} i_{2}} \in L_{\left(y_{1}, y_{2}\right)}, \quad \ell_{i_{2} j_{2}} \in L_{\left(y_{2}, y_{2}^{\prime}\right)}, \quad \ell_{i_{1} j_{1}} \in L_{\left(y_{1}, y_{1}^{\prime}\right)}, \quad \ell_{j_{1} j_{2}} \in L_{\left(y_{1}^{\prime}, y_{2}^{\prime}\right)}
$$

are given by $G_{i_{1} j_{1} i_{2} j_{2}}$-orbits of

$$
\begin{array}{ll}
\hat{\ell}_{i_{1} i_{2}}=\left((g, h),\left[\tilde{\gamma}_{i_{1}}, \tilde{\gamma}_{i_{2}}, u_{i_{1} i_{2}}\right]_{i_{1} i_{2}}\right), & \hat{\ell}_{i_{2} j_{2}}=\left((g, h),\left[\tilde{\gamma}_{i_{2}}, \tilde{\gamma}_{j_{2}}, u_{i_{2} j_{2}}\right]_{i_{2} j_{2}}\right), \\
\hat{\ell}_{i_{1} j_{1}}=\left((g, h),\left[\tilde{\gamma}_{i_{1}}, \tilde{\gamma}_{j_{1}}, u_{i_{1} j_{1}}\right]_{i_{1} j_{1}}\right), & \hat{\ell}_{j_{1} j_{2}}=\left((g, h),\left[\tilde{\gamma}_{j_{1}}, \tilde{\gamma}_{j_{2}}, u_{j_{1} j_{2}}\right]_{j_{1} j_{2}}\right) .
\end{array}
$$

with $\mu\left(\ell_{i_{1} i_{2}} \otimes \ell_{i_{2} j_{2}}\right)=\mu\left(\ell_{i_{1} j_{1}} \otimes \ell_{j_{1} j_{2}}\right)$, i.e.

$$
u_{i_{1} i_{2}} u_{i_{2} j_{2}}=u_{i_{1} j_{1}} u_{j_{1} j_{2}} .
$$

The bundle isomorphism $\alpha$ of (7.38) will be generated by a map $\tilde{\alpha}$ such that

$$
\tilde{\alpha}\left(\tilde{\gamma}, \ell_{i_{1} i_{2}} \otimes \ell_{i_{2} j_{2}}\right)=\left(\tilde{\gamma}, \ell_{i_{1} j_{1}} \otimes \ell_{j_{1} j_{2}}\right) .
$$

Consistency requires that $\tilde{\alpha}$ commutes with the action of $\tilde{Z}$, i.e. that

$$
\tilde{\alpha}\left(z \tilde{\gamma}, \ell_{i_{1} i_{2}} \star z^{-1} \otimes \ell_{i_{2} j_{2}} \cdot z^{-1}\right)=\left(\tilde{\gamma}, \ell_{i_{1} j_{1}} \cdot z^{-1} \otimes \ell_{j_{1} j_{2}}\right) .
$$

In view of Eqs. (7.26), (7.34) and (7.44), this is guaranteed by the relation

$$
\chi_{i_{1} i_{2}}(\tilde{z})^{-k} \chi_{i_{2}}(\tilde{z})^{k} \chi(z)=\chi_{i_{1}}(\tilde{z})^{k} \chi(z),
$$

which follows from identity (7.12). That the bundle isomorphism $\alpha$ preserves the connections follows from the equality of the (modified) connection forms

$$
\tilde{\gamma}^{*} \hat{A}_{i_{1} i_{2}}+\hat{A}_{i_{2} j_{2}}+\hat{A}_{i_{2}}=\hat{A}_{i_{1} j_{1}}+\hat{A}_{i_{1}}+\hat{A}_{j_{1} j_{2}}
$$

which is easy to check.

For the bundle isomorphism $\alpha$ to define a gerbe 1-isomorphism from $\left(\mathcal{G}_{k}\right)_{12}$ to $\mathcal{I}_{\rho_{k}} \otimes$ $\left(\mathcal{G}_{k}\right)_{2}$, one has to require a proper behavior with respect to the groupoid multiplication 
[26]. More precisely, what is needed is the coincidence of two composed isomorphisms of line bundles over $W_{12}^{[3]}$. The first one is

$$
\begin{gathered}
\left(L_{12}\right)_{1,2} \otimes\left(L_{12}\right)_{2,3} \otimes E_{3} \stackrel{I d \otimes \alpha_{2,3}}{\longrightarrow}\left(L_{12}\right)_{1,2} \otimes E_{2} \otimes\left(L_{2}\right)_{2,3} \\
\stackrel{\alpha_{1,2} \otimes I d}{\longrightarrow} E_{1} \otimes\left(L_{2}\right)_{1,2} \otimes\left(L_{2}\right)_{2,3} \stackrel{I d \otimes\left(\mu_{2}\right)_{1,2,3}}{\longrightarrow} E_{1} \otimes\left(L_{2}\right)_{1,3},
\end{gathered}
$$

with the exterior subscripts referring to the components of $W_{12}^{[3]}$. The second one is

$$
\left(L_{12}\right)_{1,2} \otimes\left(L_{12}\right)_{2,3} \times E_{3} \stackrel{\left(\mu_{12}\right)_{1,2,3} \otimes I d}{\longrightarrow}\left(L_{12}\right)_{1,3} \otimes E_{3} \stackrel{\alpha_{1,3}}{\longrightarrow} E_{1} \otimes\left(L_{2}\right)_{1,3} .
$$

Straightforward verification that they coincide is carried out in Appendix 7.

7.3. Construction of 2-isomorphism $\beta$. 2-isomorphism $\beta$ of Definition 5.1 compares 1 -isomorphisms of gerbes over $\Gamma^{2} \times \tilde{G}$. First, consider gerbe $\left(\mathcal{G}_{k}\right)_{123}=\left(Y_{123}, B_{123}\right.$, $\left.L_{123}, \mu_{123}\right)$. The same way as before for $Y_{12}$, we shall use the map

$$
\tilde{G}^{2} \times Y \ni\left(\tilde{\gamma}_{1}, \tilde{\gamma}_{2}, y\right) \longmapsto A d_{\tilde{\gamma}_{1} \tilde{\gamma}_{2}} y \in Y
$$

constant on orbits of the $\tilde{Z}^{2}$-action

$$
\left(\tilde{\gamma}_{1}, \tilde{\gamma}_{2}, y\right) \longmapsto\left(z_{1} \tilde{\gamma}_{1}, z_{2} \tilde{\gamma}_{2}, y\left(z_{1} z_{2}\right)^{-1}\right)
$$

in order to identify

$$
Y_{123}^{[n]} \cong\left(\tilde{G}^{2} \times Y^{[n]}\right) / \tilde{Z}^{2}
$$

As line bundles,

$$
L_{123} \cong\left(\tilde{G}^{2} \times L\right) / \tilde{Z}^{2}
$$

for the action

$$
\left(\tilde{\gamma}_{1}, \tilde{\gamma}_{2}, \ell_{i j}\right) \longmapsto\left(z_{1} \tilde{\gamma}_{1}, z_{2} \tilde{\gamma}_{2}, \ell_{i j} \star\left(z_{1} z_{2}\right)^{-1}\right)
$$

The connection in $L$ has to be modified by twisting the flat structure of $\tilde{G}^{2} \times \hat{L}_{i j}$ by the connection 1 -form $\left(\tilde{\gamma}_{1} \tilde{\gamma}_{2}\right)^{*} \hat{A}_{i j}$, see Eq. (7.28). Similarly, for gerbe $\left(\mathcal{G}_{k}\right)_{23}=$ $\left(Y_{23}, B_{23}, L_{23}, \mu_{23}\right)$ over $\Gamma^{2} \times \tilde{G}$, we have:

$$
Y_{23}^{[n]} \cong\left(\tilde{G}^{2} \times Y^{[n]}\right) / \tilde{Z}^{2},
$$

where now the action of $\tilde{Z}^{2}$ is induced from the one on $\tilde{G}^{2} \times \tilde{Y}$ given by

$$
\left(\tilde{\gamma}_{1}, \tilde{\gamma}_{2}, y\right) \longmapsto\left(z_{1} \tilde{\gamma}_{1}, z_{2} \tilde{\gamma}_{2}, y z_{2}^{-1}\right) .
$$

As line bundles,

$$
L_{23} \cong\left(\tilde{G}^{2} \times L\right) / \tilde{Z}^{2}
$$

for the action

$$
\left(\tilde{\gamma}_{1}, \tilde{\gamma}_{2}, \ell_{i j}\right) \longmapsto\left(z_{1} \tilde{\gamma}_{1}, z_{2} \tilde{\gamma}_{2}, \ell_{i j} \star z_{2}^{-1}\right),
$$


with the connection of $\tilde{G}^{2} \times L$ modified now using 1-forms $\tilde{\gamma}_{2}^{*} \hat{A}_{i j}$. Finally, for gerbe $\left(\mathcal{G}_{k}\right)_{3}=\left(Y_{3}, B_{3}, L_{3}, \mu_{3}\right)$,

$$
Y_{3}^{[n]} \cong\left(\tilde{G}^{2} \times Y^{[n]}\right) / \tilde{Z}^{2} \text { and } L_{3} \cong\left(\tilde{G}^{2} \times L\right) / \tilde{Z}^{2},
$$

with $\tilde{Z}^{2}$ acting only on the factors $\tilde{G}^{2}$.

For the fiber-product space $W_{123}=Y_{123} \times_{\left(\Gamma^{2} \times \tilde{G}\right)} Y_{23} \times_{\left(\Gamma^{2} \times \tilde{G}\right)} Y_{3}$, we have

$$
W_{123}=\left(\tilde{G}^{2} \times Y^{[3]}\right) / \tilde{Z}^{2}
$$

for the action

$$
\left(\tilde{\gamma}_{1}, \tilde{\gamma}_{2},\left(y, y^{\prime}, y^{\prime \prime}\right)\right) \longmapsto\left(z_{1} \tilde{\gamma}_{1}, z_{2} \tilde{\gamma}_{2},\left(y\left(z_{1} z_{2}\right)^{-1}, y^{\prime} z_{2}^{-1}, y^{\prime \prime}\right)\right)
$$

of $\tilde{Z}^{2}$. We may pull back the line bundle $E$ over $W_{12}$ in three different ways to $W_{123}$, obtaining the respective line bundles $E_{1,23}, E_{2,3}$ and $E_{12,3}$. One has

$$
\begin{gathered}
E_{1,23} \cong\left(\tilde{G}^{2} \times L_{1,2}\right) / \tilde{Z}^{2}, \quad E_{2,3} \cong\left(\tilde{G}^{2} \times L_{2,3}\right) / \tilde{Z}^{2}, \\
E_{12,3} \cong\left(\tilde{G}^{2} \times L_{1,3}\right) / \tilde{Z}^{2} .
\end{gathered}
$$

The actions of $\tilde{Z}^{2}$ above are defined as follows. If $\left(y, y^{\prime}, y^{\prime \prime}\right) \in Y_{i j k} \subset Y^{[3]}$ and $\ell_{i j}, \ell_{j k}$ and $\ell_{i k}$ are as in (7.17), i.e. $\ell_{i j} \in L_{\left(y, y^{\prime}\right)} \subset L_{1,2}, \ell_{j k} \in L_{\left(y^{\prime}, y^{\prime \prime}\right)} \subset L_{2,3}$ and $\ell_{i k} \in L_{\left(y, y^{\prime \prime}\right)} \subset L_{1,3}$, then under $\left(z_{1}, z_{2}\right) \in \tilde{Z}^{2}$,

$$
\begin{aligned}
& \left(\tilde{\gamma}_{1}, \tilde{\gamma}_{2}, \ell_{i j}\right) \longmapsto\left(z_{1} \tilde{\gamma}_{1}, z_{2} \tilde{\gamma}_{2},\left(\ell_{i j} \star z_{2}^{-1}\right) \cdot z_{1}^{-1}\right), \\
& \left(\tilde{\gamma}_{1}, \tilde{\gamma}_{2}, \ell_{j k}\right) \longmapsto\left(z_{1} \tilde{\gamma}_{1}, z_{2} \tilde{\gamma}_{2}, \ell_{j k} \cdot z_{2}^{-1}\right), \\
& \left(\tilde{\gamma}_{1}, \tilde{\gamma}_{2}, \ell_{i k}\right) \longmapsto\left(z_{1} \tilde{\gamma}_{1}, z_{2} \tilde{\gamma}_{2}, \ell_{i k} \cdot\left(z_{1} z_{2}\right)^{-1}\right) .
\end{aligned}
$$

The connection of $L$ in the three pullbacks in (7.63) has to be modified by twisting the flat structure of $\tilde{G}^{2} \times \hat{L}_{i j}$ by the 1 -form

$$
\hat{A}_{i j}^{1,23}=i k \operatorname{tr}\left(\tau_{j}-\tau_{i}\right) \Theta\left(\tilde{\gamma}_{2} h_{g}\right)+i k \operatorname{tr} A d_{\tilde{\gamma}_{2} h_{g}}\left(\tau-\tau_{i}\right) \Theta\left(\tilde{\gamma}_{1}\right),
$$

that of $\tilde{G}^{2} \times \hat{L}_{j k}$ by

$$
\hat{A}_{j k}^{2,3}=i k \operatorname{tr}\left(\tau_{k}-\tau_{j}\right) \Theta\left(h_{g}\right)+i k \operatorname{tr} A d_{h_{g}}\left(\tau-\tau_{j}\right) \Theta\left(\tilde{\gamma}_{2}\right),
$$

and that of $\tilde{G}^{2} \times \hat{L}_{i k}$ by

$$
\hat{A}_{i k}^{12,3}=i k \operatorname{tr}\left(\tau_{k}-\tau_{i}\right) \Theta\left(h_{g}\right)+i k \operatorname{tr} A d_{h_{g}}\left(\tau-\tau_{i}\right) \Theta\left(\tilde{\gamma}_{1} \tilde{\gamma}_{2}\right) .
$$

There is a natural isomorphism $\beta: E_{1,23} \otimes E_{2,3} \rightarrow E_{12,3}$ given by the groupoid multiplication $\mu$ in $L$, i.e. induced by the map

$$
\left(\tilde{\gamma}_{1}, \tilde{\gamma}_{2}, \ell_{i j} \otimes \ell_{j k}\right) \stackrel{\tilde{\beta}}{\longrightarrow}\left(\tilde{\gamma}_{1}, \tilde{\gamma}_{2}, \mu\left(\ell_{i j} \otimes \ell_{j k}\right)\right) .
$$

Indeed, $\tilde{\beta}$ commutes with the action of $\tilde{Z}^{2}$ because $\mu\left(\left(\ell_{i j} \star z_{2}^{-1}\right) \cdot z_{1}^{-1} \otimes \ell_{j k} \cdot z_{2}^{-1}\right)=$ $\ell_{i k} \cdot\left(z_{1} z_{2}\right)^{-1}$ if $\mu\left(\ell_{i j} \otimes \ell_{j k}\right)=\ell_{i k}$ as

$$
\chi_{i}\left(\tilde{z}_{1}\right)^{k} \chi\left(z_{1}\right) \chi_{i j}\left(\tilde{z}_{2}\right)^{-k} \chi_{j}\left(\tilde{z}_{2}\right)^{k} \chi\left(z_{2}\right)=\chi_{i}\left(\tilde{z}_{1} \tilde{z}_{2}\right)^{k} \chi\left(z_{1} z_{2}\right) .
$$


Besides, $\tilde{\beta}$ intertwines the modified connections since

$$
\hat{A}_{i j}^{1,23}+\hat{A}_{j k}^{2,3}=\hat{A}_{i k}^{12,3},
$$

as a short calculation shows.

For the line bundle isomorphism $\beta$ to provide a gerbe 2-isomorphism required by Definition 5.1, one needs (see [26]) that over

$$
W_{123}^{[2]} \cong\left(\tilde{G}^{2} \times Y^{[6]}\right) / \tilde{Z}^{2},
$$

with the action of $\tilde{Z}^{2}$ induced from that in (7.62), the diagram of line bundle isomorphisms

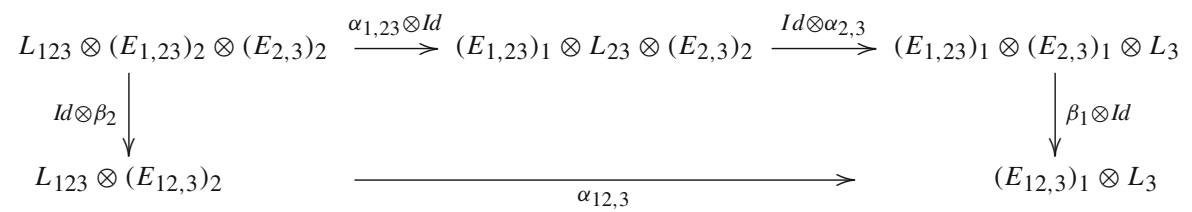

with the exterior subscripts referring to the pullbacks to $W_{123}^{[2]}$ and with the obvious pullbacks omitted, be commutative. This is checked in Appendix 8.

7.4. Commutativity of diagram (5.1). This is the identity

$$
\beta_{1,23,4} \bullet\left(\left(I d \otimes \beta_{2,3,4}\right) \circ I d\right)=\beta_{12,3,4} \bullet\left(I d \circ \beta_{1,2,34)}\right)
$$

for composed 2-isomorphisms between 1-isomorphisms of gerbes over $\Gamma^{2} \times \tilde{G}$ (see [54] for the abstract definition of the vertical $\bullet$ and horizontal $\circ$ compositions of 2-morphisms). The left- and the right-hand side are the following compositions of the isomorphisms of line bundles:

$$
E_{1,234} \otimes E_{2,34} \otimes E_{3,4} \stackrel{I d \otimes \beta_{2,3,4}}{\longrightarrow} E_{1,234} \otimes E_{23,4} \stackrel{\beta_{1,23,4}}{\longrightarrow} E_{123,4},
$$

and

$$
E_{1,234} \otimes E_{2,34} \otimes E_{3,4} \stackrel{\beta_{1,2,34} \otimes I d}{\longrightarrow} E_{12,34} \otimes E_{3,4} \stackrel{\beta_{12,3,4}}{\longrightarrow} E_{123,4}
$$

respectively, over the fiber-product space $W_{1234}=(Y)_{1234} \times{ }_{\left(\Gamma^{3} \times \tilde{G}\right)}(Y)_{234} \times_{\left(\Gamma^{3} \times \tilde{G}\right)}$ $(Y)_{34} \times{ }_{\left(\Gamma^{3} \times \tilde{G}\right)}(Y)_{4}$. It is checked in Appendix 9 that they coincide. This proves identity (7.75) establishing the commutativity of diagram (5.1) of Definition 5.1 and completing the construction of $\Gamma$-equivariant structures on gerbe $\mathcal{G}_{k}$ over $\tilde{G}$ for the adjoint action of $\Gamma=\tilde{G} / \tilde{Z}$ on $\tilde{G}$.

Theorem 7.1. The $\Gamma$-equivariant structures on the WZW gerbe $\mathcal{G}_{k}$ over $\tilde{G}$ constructed above are non-isomorphic for different characters $\chi: \tilde{Z} \rightarrow U(1)$ and each $\Gamma$-equivariant structure on $\mathcal{G}_{k}$ is isomorphic to one of them. 
Proof. The general discussion of classification of $\Gamma$-equivariant structures in Sect. 6.5 showed that different isomorphism classes of $\Gamma$-equivariant structures correspond in this case to cohomology classes $\left[b^{\prime}\right] \in H^{1}(\Gamma \times M, U(1)) \cong H^{1}(\Gamma, U(1))$ in the image of homomorphism $\kappa$, see Corollary 6.15. The classes $\left[b^{\prime}\right]$ are the isomorphism classes of flat line bundles over $\Gamma$ by which differ the line bundles $E$ over $W_{12}$ involved in the above construction of 1-isomorphisms $\alpha$ of Definition 5.1. Different choices of characters $\chi: \tilde{Z} \rightarrow U(1)$ correspond to tensoring $E$ with such flat line bundles, as was remarked in Sect. 7.2. The claim of the theorem now follows from the isomorphism of $H^{1}(\Gamma, U(1))$ with the character group $\tilde{Z}^{*}$.

Remark 7.2. It is natural to conjecture that the special $\Gamma$-equivariant structure for which Eq. (6.76) gives the contributions of the topologically non-trivial gauge fields to the partition function of the $\tilde{G} / \Gamma$ coset theory corresponds to $\chi=1$.

\section{Conclusions}

We revisited the problem of the gauging of rigid symmetries in two-dimensional sigma models with the Wess-Zumino action related to a closed 3-forms $H$ on the target manifold. For topologically trivial gauge fields given by global Lie-algebra valued 1-forms on the worldsheet, the gauging prescription of Refs. [37] and [36], recalled in Sect. 3.1, assures infinitesimal gauge invariance. We showed, however, that it may lead to global gauge anomalies. In Corollary 4.5 and Sect. 6.1, we classified such anomalies using geometric tools based on the theory of bundle gerbes. As was shown in Sect. 4.2, global gauge anomalies occur, for example, in numerous WZW sigma models with non-simply connected target groups when one gauges their adjoint symmetries. They lead to the inconsistency, discussed in Sect. 4.4, of the corresponding coset models obtained by integrating out the external gauge fields in the respective gauged WZW models. In Sect. 5.1, we introduced geometric structures called equivariant gerbes, living on the target space, that permit an anomaly-free coupling of WZ amplitudes to arbitrary (also topologically non-trivial) gauge fields. A detailed analysis of obstructions to the existence of such structures was performed and their classification was obtained in Sect. 6. In particular, we proved Theorem 6.9 asserting that the gerbes relevant to the WZW theories with compact semi-simple target groups can be equipped with equivariant structures with respect to adjoint symmetries if and only if there is no global gauge anomaly in the coupling of the WZW model to topologically trivial gauge fields. In Sect. 7, we explicitly constructed all nonequivalent equivariant structures in the case of simply connected target groups. Different equivariant structures result in the coupling to topologically non-trivial gauge fields that differs by phases. We showed in Sect. 6.6 that such ambiguities, anticipated in [33], are given by characters of a subgroup of the fundamental group of the symmetry group, if the latter is connected, see Corollary 6.20. In Sect. 6.7, we discussed how such ambiguities are reflected in the (fixed-poit resolved) partition functions of the non-anomalous coset theories. We do not know if, in general, the existence of equivariant gerbes is also a necessary condition for the existence of non-anomalous coupling of WZ amplitudes to gauge fields in topologically non-trivial sectors, but this is a plausible conjecture. The analysis of the present paper was limited to the case of oriented closed worldsheets. Local gauge anomalies on worldsheets with boundary were studied in [12]. A generalization of the present work to the case of such worldsheets, or worldsheets with conformal defects, will be discussed in a separate publication. An extension of WZ amplitudes to unoriented surfaces requires an additional structure on 
gerbes that was introduced under the name of Jandl structure in [48], see also [26,27]. We plan to discuss the interrelation between equivariant structures, Jandl structures, and multiplicative structures on gerbes of $[5,25,55]$, in a future study, with applications to orientifolds of coset models. Other possible extensions of our work should cover the cases of WZW and coset theories with gauging of twisted-adjoint symmetries or with non-compact targets, of supersymmetric sigma models, and applications to global aspects of $T$-duality [34]. It should also be possible to study global gauge anomalies for higher dimensional WZ actions on spacetimes with arbitrary topology using the theory of bundle $n$-gerbes [6].

Acknowledgements. The authors acknowledge the support of the contract ANR-05-BLAN-0029-03 in the early stage of this collaboration. The work of K.W. was supported by a Feodor-Lynen scholarship granted by the Alexander von Humboldt Foundation. That of R.R.S. was partially funded by the Collaborative Research Centre 676 "Particles, Strings and the Early Universe - the Structure of Matter and Space-Time".

Open Access This article is distributed under the terms of the Creative Commons Attribution Noncommercial License which permits any noncommercial use, distribution, and reproduction in any medium, provided the original author(s) and source are credited.

\section{Appendices}

1. Proof of Proposition 3.1. We have to find conditions under which the coupled amplitudes $\boldsymbol{A}(\varphi, A)$ given by Eq. (3.5) are invariant under infinitesimal gauge transformations. Setting $\mathrm{e}^{-t \Lambda} \phi=\left(I d, \mathrm{e}^{-t \Lambda} \varphi\right)$ and denoting by $\bar{\Lambda}$ the vector field on $\Sigma \times M$ in the direction of $M$ given by $\bar{\Lambda}(x, m)=\left.\frac{d}{d t}\right|_{t=0}\left(x, \mathrm{e}^{-t \Lambda} m\right)$, we observe that

$$
\begin{aligned}
& \left.\frac{d}{d t}\right|_{t=0} \int_{\Sigma}\left(\mathrm{e}^{-t \Lambda} \phi\right)^{*}\left(-v(A)+\frac{1}{2} u\left(A^{2}\right)\right)=\int_{\Sigma} \phi^{*} \mathcal{L}_{\bar{\Lambda}}\left(-v(A)+\frac{1}{2} u\left(A^{2}\right)\right) \\
& \quad=\int_{\Sigma} \phi^{*} \iota_{\bar{\Lambda}} d\left(-v(A)+\frac{1}{2} u\left(A^{2}\right)\right)
\end{aligned}
$$

since the other term $d_{\iota}$ in the Lie derivative gives a term that vanishes by integration by parts. Similarly, as $\left.\frac{d}{d t}\right|_{t=0} \mathrm{e}^{-t \Lambda} A=d \Lambda-[\Lambda, A]$, see Eq. (3.4), one obtains

$$
\begin{aligned}
& \left.\frac{d}{d t}\right|_{t=0} \int_{\Sigma} \phi^{*}\left(-v\left(\mathrm{e}^{-t \Lambda} A\right)+\frac{1}{2} u\left(\left(\mathrm{e}^{-t \Lambda} A\right)^{2}\right)\right) \\
& =\int_{\Sigma} \phi^{*}(-v(d \Lambda-[\Lambda, A])+u((d \Lambda-[\Lambda, A]) A)) .
\end{aligned}
$$

On the other hand, $\boldsymbol{A}_{W Z}\left(\mathrm{e}^{-t \Lambda} \varphi\right)=H_{o l} \mathcal{G}_{2}\left(\mathrm{e}^{-t \Lambda} \phi\right)$, where the subscript 2 on $\mathcal{G}$ refers to the pullback along the projection from $\Sigma \times M$ to $M$ (the latter relation follows from the behavior of gerbe holonomy under gerbe pullbacks). Proceeding as in the proof of Proposition 5.5 one then shows that

$$
\left.\frac{d}{d t}\right|_{t=0} \boldsymbol{A}_{W Z}\left(\mathrm{e}^{-t \Lambda} \varphi\right)=\left(i \int_{\Sigma} \phi^{*}\left(\iota_{\bar{\Lambda}} H\right)\right) \boldsymbol{A}_{W Z}(\varphi),
$$


so that $\iota_{\bar{\Lambda}} H$ (more pedantically defined as $\iota_{\bar{\Lambda}} H_{2}$ ) is a form on $\Sigma \times M$. Gathering the above relations, we infer that

$$
\begin{aligned}
& \left.\frac{d}{d t}\right|_{t=0} \boldsymbol{A}_{W Z}\left(\mathrm{e}^{-t \Lambda} \varphi, \mathrm{e}^{-t \Lambda} A\right)=\left(i \int _ { \Sigma } \phi ^ { * } \left[\iota_{\bar{\Lambda}}\left(H+d\left(-v(A)+\frac{1}{2} u\left(A^{2}\right)\right)\right)\right.\right. \\
& -v(d \Lambda-[\Lambda, A])+u((d \Lambda-[\Lambda, A]) A)]) A_{W Z}(\varphi, A) .
\end{aligned}
$$

Consequently, the invariance of the amplitudes $\boldsymbol{A}_{W Z}(\varphi, A)$ under infinitesimal gauge transformations requires that for all $\varphi$ and $A$,

$$
\int_{\Sigma} \phi^{*}\left[\iota_{\bar{\Lambda}}\left(H+d\left(-v(A)+\frac{1}{2} u\left(A^{2}\right)\right)\right)-v(d \Lambda-[\Lambda, A])+u((d \Lambda-[\Lambda, A]) A)\right]=0 .
$$

In order to proceed, it will be easier to employ a basis $\left(t^{a}\right)$ in $\mathbf{g}$, writing $A=t^{a} A^{a}, \Lambda=$ $t^{a} \Lambda^{a}$ and using the notations of Remark 3.2.2, Eq. (A.1.5) may then be rewritten as

$$
\begin{aligned}
\int_{\Sigma} \phi^{*} & {\left[\Lambda^{a}\left(\iota^{a}\left(H+d\left(-v^{b} A^{b}+\frac{1}{2} u^{b c} A^{b} A^{c}\right)\right)+f^{a b c}\left(v^{c} A^{b}-u^{c d} A^{b} A^{d}\right)\right)\right.} \\
& \left.+\left(d \Lambda^{a}\right)\left(v^{a}+u^{a b} A^{b}\right)\right] \\
= & \int_{\Sigma} \phi^{*}\left[\Lambda ^ { a } \left(\iota^{a} H-\iota^{a}\left(d v^{b}\right) A^{b}+\iota^{a} v^{b} d A^{b}+\frac{1}{2} \iota^{a}\left(d u^{b c}\right) A^{b} A^{c}+f^{a b c} v^{c} A^{b}\right.\right. \\
& \left.\left.-f^{a b c} u^{c d} A^{b} A^{d}-d v^{a}-\left(d u^{a b}\right) A^{b}-u^{a b} d A^{b}\right)\right]=0,
\end{aligned}
$$

where the terms in the last line were obtained by integration by parts. Since $\Lambda^{a}$ are arbitrary functions on $\Sigma$, we infer that the 2-form

$$
\begin{gathered}
\varphi^{*}\left(\iota^{a} H-d v^{a}\right)+\varphi^{*}\left(-\iota^{a}\left(d v^{b}\right)+f^{a b c} v^{c}-d u^{a b}\right) A^{b}+\varphi^{*}\left(\iota^{a} v^{b}-u^{a b}\right) d A^{b} \\
+\frac{1}{2} \varphi^{*}\left(\iota^{a} d u^{b c}-f^{a b d} u^{d c}+f^{a c d} u^{d b}\right) A^{b} A^{c}
\end{gathered}
$$

on $\Sigma$ has to vanish for all maps $\varphi: \Sigma \rightarrow M$ and all 1-forms $A^{a}$ on $\Sigma$. It is easy to see that this imposes the separate constraints

$$
\begin{gathered}
\iota^{a} H-d v^{a}=0, \quad-\iota^{a}\left(d v^{b}\right)+f^{a b c} v^{c}-d u^{a b}=0, \\
\iota^{a} v^{b}-u^{a b}=0, \quad \iota^{a} d u^{b c}-f^{a b d} u^{d c}+f^{a c d} u^{d b}=0 .
\end{gathered}
$$

The $1^{\text {st }}$ of these equalities gives the left of Eqs. (3.6). The $3^{\text {rd }}$ one gives Eq. (3.7), implying also the right of Eqs. (3.6) and, via the $2^{\text {nd }}$ equality, the middle of Eqs. (3.6). The $4^{\text {th }}$ equality may be rewritten as

$$
\iota_{\bar{X}} d \iota_{\bar{Y}} v(Z)-\iota_{[\bar{X}, \bar{Y}]} v(Z)+\iota_{[\bar{X}, \bar{Z}]} v(Y)=0
$$

and now holds automatically since

$$
\iota_{\bar{X}} d \iota_{\bar{Y}} v(Z)=\mathcal{L}_{\bar{X}} \iota_{\bar{Y}} v(Z), \quad \iota_{[\bar{X}, \bar{Z}]} v(Y)=-\iota_{\bar{Y}} v([X, Z])=-\iota_{\bar{Y}} \mathcal{L}_{\bar{X}} v(Z)
$$

and $\left[\mathcal{L}_{\bar{X}}, \iota_{\bar{Y}}\right]=\iota_{[\bar{X}, \bar{Y}]}$. This ends the proof of Proposition 3.1. 
2. Proof of Lemma 3.13. In order to prove that the 2 -form $\rho$ of Eq. (3.26) is $\Gamma$-invariant, recall that $\Gamma \times M$ is considered as a $\Gamma$-space with the action $\tilde{\ell}_{\gamma}\left(\gamma^{\prime}, m\right)=\left(A d_{\gamma}\left(\gamma^{\prime}\right), \ell_{\gamma} m\right)$ of $\gamma \in \Gamma$. Using relation (4.5), we obtain:

$$
\begin{aligned}
\tilde{\ell}_{\gamma}^{*} \rho & =\tilde{\ell}_{\gamma}^{*}\left(-v(\Theta)+\frac{1}{2}\left(\iota_{\bar{\Theta}} v\right)(\Theta)\right) \\
& =-\left(\ell_{\gamma}^{*} v\right)\left(A d_{\gamma}^{*} \Theta\right)+\frac{1}{2}\left(l \frac{A d_{\gamma^{-1}}\left(A d_{\gamma}^{*} \Theta\right)}{\ell_{\gamma}^{*}} v\right)\left(A d_{\gamma}^{*} \Theta\right) \\
& =-\left(v\left(A d_{\gamma^{-1}}\left(A d_{\gamma}^{*} \Theta\right)\right)+\frac{1}{2}\left(l \overline{A d_{\gamma^{-1}}\left(A d_{\gamma}^{*} \Theta\right)} v\right)\left(A d_{\gamma^{-1}}\left(A d_{\gamma}^{*} \Theta\right)\right),\right.
\end{aligned}
$$

where the $2^{\text {nd }}$ equality follows from the $2^{\text {nd }}$ of relations (3.24). The identity $A d_{\gamma}^{*} \Theta=$ $A d_{\gamma}(\Theta)$ implies that the right-hand side is $\gamma$-independent so that the $\Gamma$-invariance of $\rho$ follows.

Let us pass to the proof of relation (3.31). Using the equality $\Theta\left(\gamma_{1} \gamma_{2}\right)=A d_{\gamma_{2}^{-1}} \Theta\left(\gamma_{1}\right)+$ $\Theta\left(\gamma_{2}\right)$, we obtain on $\Gamma^{2} \times M$,

$$
\begin{aligned}
& \rho_{12}\left(\gamma_{1}, \gamma_{2}, m\right)=\rho\left(\gamma_{1} \gamma_{2}, m\right) \\
& =-\left[v\left(A d_{\gamma_{2}^{-1}} \Theta\left(\gamma_{1}\right)\right)\right](m)-\left[v\left(\Theta\left(\gamma_{2}\right)\right)\right](m)+\frac{1}{2}\left[l \frac{}{A d_{\gamma_{2}^{-1}} \Theta\left(\gamma_{1}\right)} v\left(A d_{\gamma_{2}^{-1}} \Theta\left(\gamma_{1}\right)\right)\right](m) \\
& +\frac{1}{2}\left[\iota_{\Theta\left(\gamma_{2}\right)} v\left(A d_{\gamma_{2}^{-1}} \Theta\left(\gamma_{1}\right)\right)\right](m)+\frac{1}{2}\left[\iota \frac{}{A d_{\gamma_{2}^{-1}} \Theta\left(\gamma_{1}\right)} v\left(\Theta\left(\gamma_{2}\right)\right)\right](m) \\
& +\frac{1}{2}\left[\iota_{\bar{\Theta}\left(\gamma_{2}\right)} v\left(\Theta\left(\gamma_{2}\right)\right)\right](m) \text {. }
\end{aligned}
$$

Using, again, the $2^{\text {nd }}$ of relations (3.24) as well as the last of equalities (3.6), identity (4.5) and, finally, equality (3.27), we may rewrite the last identity as

$$
\begin{aligned}
\rho_{12} & \left(\gamma_{1}, \gamma_{2}, m\right) \\
= & -\left[\ell_{\gamma_{2}}^{*} v\left(\Theta\left(\gamma_{1}\right)\right)\right](m)-\left[v\left(\Theta\left(\gamma_{2}\right)\right)\right](m)+\frac{1}{2}\left[\ell_{\gamma_{2}}^{*}\left(\iota_{\bar{\Theta}\left(\gamma_{1}\right)} v\left(\Theta\left(\gamma_{1}\right)\right)\right)\right](m) \\
& +\left[\iota_{\bar{\Theta}\left(\gamma_{2}\right)} \ell_{\gamma_{2}}^{*} v\left(\Theta\left(\gamma_{1}\right)\right)\right](m)+\frac{1}{2}\left[\iota_{\bar{\Theta}\left(\gamma_{2}\right)} v\left(\Theta\left(\gamma_{2}\right)\right)\right](m) \\
= & {\left[\exp \left[-\iota_{\bar{\Theta}\left(\gamma_{2}\right)} \ell_{\gamma_{2}}^{*}\left(-v\left(\Theta\left(\gamma_{1}\right)\right)+\frac{1}{2} \iota_{\bar{\Theta}\left(\gamma_{1}\right)} v\left(\Theta\left(\gamma_{1}\right)\right)\right)\right](m)-\left[v\left(\Theta\left(\gamma_{2}\right)\right)\right](m)\right.} \\
& +\frac{1}{2}\left[\iota_{\bar{\Theta}\left(\gamma_{2}\right)} v\left(\Theta\left(\gamma_{2}\right)\right)\right](m) \\
= & {\left[-v\left(\Theta\left(\gamma_{1}\right)\right)+\frac{1}{2} \iota_{\bar{\Theta}\left(\gamma_{1}\right)} v\left(\Theta\left(\gamma_{1}\right)\right)\right]\left(\gamma_{2} m\right)+\rho\left(\gamma_{2}, m\right) } \\
= & \rho\left(\gamma_{1}, \gamma_{2} m\right)+\rho\left(\gamma_{2}, m\right)=\left[\rho_{1,23}+\rho_{2,3}\right]\left(\gamma_{1}, \gamma_{2}, m\right) .
\end{aligned}
$$

3. Proof of Proposition 4.2. Note, first, that the action $L_{h}$ of the gauge transformation $h$ on $\Sigma \times M$ defined in (4.1) may be factored through $\Sigma \times \Gamma \times M$ as

$$
(x, m) \stackrel{K_{h}}{\longrightarrow}(x, h(x), m) \stackrel{I d \times \ell}{\longrightarrow}(x, h(x) m) .
$$

It follows that

$$
L_{h}^{*} \mathcal{G}_{A}=K_{h}^{*}(I d \times \ell)^{*} \mathcal{G}_{A}=K_{h}^{*}(I d \times \ell)^{*}\left(\mathcal{I}_{\rho_{A}} \otimes \mathcal{G}_{2}\right),
$$


see the $2^{\text {nd }}$ of Eqs. (3.9). Now,

$$
(I d \times \ell)^{*}\left(\mathcal{I}_{\rho_{A}} \otimes \mathcal{G}_{2}\right)=\mathcal{I}_{\left(\rho_{A}\right)_{1,23}} \otimes \mathcal{G}_{23},
$$

with the indices referring to the factors of $\Sigma \times \Gamma \times M$ so that $\left(\rho_{A}\right)_{1,23}=(I d \times \ell)^{*} \rho_{A}$. From the definition (4.8) of the gerbe $\mathcal{F}$, it follows that

$$
\ell^{*} \mathcal{G}=\mathcal{G}_{12} \cong \mathcal{I}_{\rho} \otimes \mathcal{G}_{2} \otimes \mathcal{F}
$$

where $\cong$ stands for "is 1-isomorphic to". Equation (A.3.3) then implies

$$
(I d \times \ell)^{*}\left(\mathcal{I}_{\rho_{A}} \otimes \mathcal{G}_{2}\right) \cong \mathcal{I}_{\left(\rho_{A}\right)_{1,23}} \otimes \mathcal{I}_{\rho_{2,3}} \otimes \mathcal{G}_{3} \otimes \mathcal{F}_{2,3}=\mathcal{I}_{\left(\rho_{A}\right)_{1,23}+\rho_{2,3}} \otimes \mathcal{G}_{3} \otimes \mathcal{F}_{2,3}
$$

The substitution of this identity into the right hand side of relation (A.3.2) gives

$$
L_{h}^{*} \mathcal{G}_{A} \cong K_{h}^{*}\left(\mathcal{I}_{\left(\rho_{A}\right)_{1,23}+\rho_{2,3}} \otimes \mathcal{G}_{3} \otimes \mathcal{F}_{2,3}\right)=\mathcal{I}_{\omega} \otimes \mathcal{G}_{2} \otimes(h \times I d)^{*} \mathcal{F}
$$

where

$$
\omega:=K_{h}^{*}\left(\left(\rho_{A}\right)_{1,23}+\rho_{2,3}\right)=L_{h}^{*} \rho_{A}+(h \times I d)^{*} \rho
$$

is a 2 -form on the product space $\Sigma \times M$ that is identified in

Lemma A.3.1. $\omega=\rho_{h^{-1} A}$.

Proof. On the one hand,

$$
\begin{aligned}
& \left(L_{h}^{*} \rho_{A}\right)(x, m)=\rho_{A}(x, h(x) m)=\left[\exp \left[-\iota \overline{\left(h^{*} \Theta\right)(x)}\right] \ell_{h(x)}^{*}\left(\rho_{A}\right)(x, \cdot)\right](m) \\
& =\left[\exp \left[-\iota \overline{\left(h^{*} \Theta\right)(x)}\right] \ell_{h(x)}^{*}\left(-v(A(x))+\frac{1}{2} \iota_{\bar{A}(x)} v(A(x))\right)\right](m) \\
& =\left[-v\left(\left(A d_{h^{-1}}(A)\right)(x)\right)+\iota \frac{}{\left(h^{*} \Theta\right)(x)} v\left(\left(A d_{h^{-1}}(A)\right)(x)\right)\right.
\end{aligned}
$$

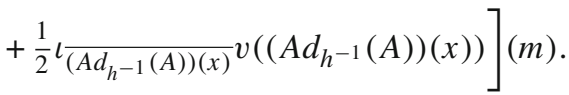

On the other hand,

$$
\left[(h \times I d)^{*} \rho\right](x, m)=\left[-v\left(h^{*} \Theta\right)+\frac{1}{2} l \frac{}{h^{*} \Theta} v\left(h^{*} \Theta\right)\right](x, m) .
$$

Adding both expressions and using the $3^{\text {rd }}$ of relations (3.6), we infer that

$$
\begin{aligned}
L_{h}^{*} \rho_{A}+(h \times I d)^{*} \rho & =-v\left(h^{*} \Theta+A d_{h^{-1}}(A)\right)+\frac{1}{2} l \frac{}{h^{*} \Theta+A d_{h^{-1}}(A)} v\left(h^{*} \Theta+A d_{h^{-1}}(A)\right) \\
& =-v\left(h^{-1} A\right)+\frac{1}{2} l \overline{h^{-1} A} v\left(h^{-1} A\right)
\end{aligned}
$$

which is the identity claimed by Lemma A.3.1.

Replacing $A$ by $h A$ and recalling definition (3.9) of the gerbe $\mathcal{G}_{A}$, we infer from Eq. (A.3.6) and Lemma A.3.1 the existence of the 1-isomorphism required by Proposition 4.2. 
4. Proof of Theorem 5.3. To prove Theorem 5.3, we shall show the existence of a canonical equivalence

$$
\operatorname{Grb}^{\nabla}(M)_{0}^{\Gamma} \cong \operatorname{Grb}^{\nabla}\left(M^{\prime}\right)
$$

of 2-categories. Here, $M$ is assumed to be a left principal $\Gamma$-bundle over $M^{\prime}$. On the left-hand side of (A.4.1) is the 2-category of $\Gamma$-equivariant gerbes over $M$ whose 2 -form $\rho$ vanishes. On the right-hand side is the 2-category of gerbes over the quotient $M^{\prime}=M / \Gamma$. We shall show that the equivalence (A.4.1) is a consequence of the fact that gerbes form a sheaf of 2-categories over smooth manifolds. We shall first recall some details about this fact.

Associated to any surjective submersion $\omega: M \rightarrow M^{\prime}$, we consider the descent 2-category Des $(\omega)$ defined as follows, with $\pi_{i_{1} \ldots i_{q}}$ standing for the projection from a $p$-fold fiber-product $M^{[p]}=M \times_{M^{\prime}} M \times_{M^{\prime}} \cdots \times{ }_{M^{\prime}} M$ to the $q$-fold fiber product $M^{[q]}$ forgetting all but the $i_{1}, \ldots, i_{q}$ components. An object in $\operatorname{Des}(\omega)$ is a triple $(\mathcal{G}, \mathcal{C}, \lambda)$ consisting of a gerbe $\mathcal{G}$ over $M$, a 1 -isomorphism $\mathcal{C}: \pi_{1}^{*} \mathcal{G} \rightarrow \pi_{2}^{*} \mathcal{G}$ over $M^{[2]}$ and a 2-isomorphism

$$
\lambda: \pi_{23}^{*} \mathcal{C} \circ \pi_{12}^{*} \mathcal{C} \Rightarrow \pi_{13}^{*} C
$$

over $M^{[3]}$ such that the diagram

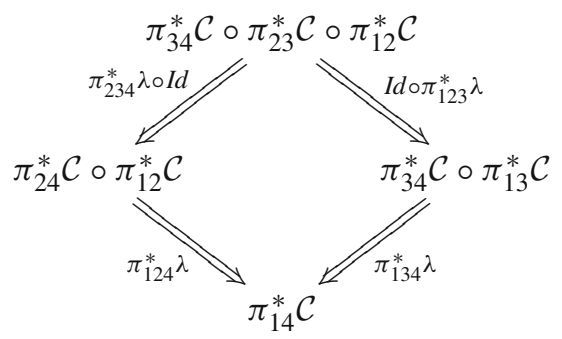

of 2-isomorphisms over $M^{[4]}$ is commutative. A 1-morphism

$$
(\mathcal{D}, \kappa):\left(\mathcal{G}^{a}, \mathcal{C}^{a}, \lambda^{a}\right) \rightarrow\left(\mathcal{G}^{b}, \mathcal{C}^{b}, \lambda^{b}\right)
$$

in $\operatorname{Des}(\omega)$ is a 1-isomorphism $\mathcal{D}: \mathcal{G}^{a} \rightarrow \mathcal{G}^{b}$ of gerbes over $M$ and a 2-isomorphism

$$
\kappa: \pi_{2}^{*} \mathcal{D} \circ \mathcal{C}^{a} \Longrightarrow \mathcal{C}^{b} \circ \pi_{1}^{*} \mathcal{D}
$$

such that the diagram

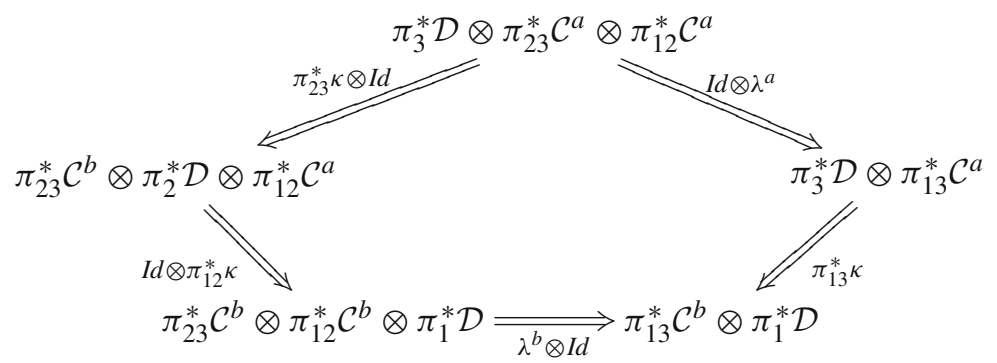


of 2-isomorphisms over $M^{[3]}$ is commutative. Finally, a 2-isomorphism $\varepsilon:(\mathcal{D}, \kappa) \Longrightarrow$ $\left(\mathcal{D}^{\prime}, \kappa^{\prime}\right)$ in $\operatorname{Des}(\omega)$ is a 2-isomorphism $\varepsilon: \mathcal{D} \Rightarrow \mathcal{D}^{\prime}$ such that the diagram

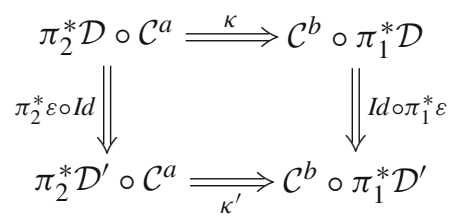

of 2-isomorphisms over $M^{[2]}$ is commutative. Composition and identities in $\operatorname{Des}(\omega)$ are defined in the natural way. There is an obvious functor

$$
\omega^{*}: G r b^{\nabla}\left(M^{\prime}\right) \rightarrow \operatorname{Des}(\omega)
$$

which sends a gerbe $\mathcal{G}$ over $M^{\prime}$ to the triple $\left(\omega^{*} \mathcal{G}, I d, I d\right)$, and is defined analogously for 1-morphisms and 2-morphisms.

An important part of the statement that gerbes form a sheaf of 2-categories over smooth manifolds is the gluing axiom for this sheaf. Using the definitions introduced above, it can be formulated in the following way.

Theorem A.4.2. For any surjective submersion $\omega: M \rightarrow M^{\prime}$, the functor (A.4.8) is an equivalence of 2-categories.

This was proven in [50], Prop. 6.7, in a setup with (bundle) gerbes without connections, but the proof actually works also for gerbes with connection.

The equivalence (A.4.1) that we have to prove is now a consequence of Theorem A.4.2 and the following relation between equivariant gerbes and the descent 2-categories introduced above. Here, we remark that the projection of any principal $G$-bundle is a surjective submersion.

Lemma A.4.3. Let $M$ be a (left) principal $\Gamma$-bundle over $M^{\prime}$ with projection $\omega: M \rightarrow M^{\prime}$. Then, there is a canonical equivalence of 2-categories

$$
\operatorname{Grb}^{\nabla}(M)_{0}^{\Gamma} \cong \operatorname{Des}(\omega)
$$

Proof. Since $M$ is a principal $\Gamma$-bundle over $M^{\prime}$, there are diffeomorphisms $f_{p}: \Gamma^{p-1} \times$ $M \rightarrow M^{[p]}$,

$$
\left(\gamma_{1}, \ldots, \gamma_{p-1}, m\right) \stackrel{f_{p}}{\longrightarrow}\left(\gamma_{1} \ldots \gamma_{p-1} m, \gamma_{2} \ldots \gamma_{p-1} m, \ldots, \gamma_{p-1} m, m\right) .
$$

The diffeomorphisms $f_{p}$ exchange various maps $\ell_{\ldots}: \Gamma^{p-1} \times M \rightarrow \Gamma^{q} \times M$ that we introduced in Sect. 3.3 with projections $\pi_{i_{1}, \ldots, i_{q}}: M^{[p]} \rightarrow M^{[q]}$ in the following way:

$$
\begin{gathered}
\ell_{12}=\pi_{1} \circ f_{2} \text { and } \ell_{2}=\pi_{2} \circ f_{2}, \\
f_{2} \circ \ell_{2,3}=\pi_{23} \circ f_{3}, \quad f_{2} \circ \ell_{1,23}=\pi_{12} \circ f_{3} \text { and } f_{2} \circ \ell_{12,3}=\pi_{13} \circ f_{3}, \\
f_{3} \circ \ell_{1,2,34}=\pi_{123} \circ f_{4}, \quad f_{3} \circ \ell_{12,3,4}=\pi_{134} \circ f_{4}, \\
f_{3} \circ \ell_{2,3,4}=\pi_{234} \circ f_{4} \text { and } f_{3} \circ \ell_{1,23,4}=\pi_{124} \circ f_{4} .
\end{gathered}
$$


Consider a descent object $(\mathcal{G}, \mathcal{C}, \lambda)$. Note that the curvature $H$ of gerbe $\mathcal{G}$ is (without any extension) $\Gamma$-equivariantly closed so that we may take $\rho=0$ for the $\Gamma$-equivariant structure on $\mathcal{G}$, see Definition 5.1. Using rules (A.4.11), the pullback of $\mathcal{C}: \pi_{1}^{*} \mathcal{G} \rightarrow \pi_{2}^{*} \mathcal{G}$ along $f_{2}: G \times M \rightarrow M^{[2]}$ is a 1-isomorphism

$$
\alpha:=f_{2}^{*} \mathcal{C}: \ell_{12}^{*} \mathcal{G} \rightarrow \ell_{2}^{*} \mathcal{G},
$$

and thus precisely the datum (i) we need for a $\Gamma$-equivariant structure. Using rules (A.4.12), the pullback of the 2-isomorphism $\lambda: \pi_{23}^{*} \mathcal{C} \circ \pi_{12}^{*} \mathcal{C} \Rightarrow \pi_{13}^{*} C$ along $f_{3}$ is a 2-isomorphism

$$
\beta:=f_{3}^{*} \lambda: \ell_{2,3}^{*} \alpha \circ \ell_{1,23}^{*} \alpha \Rightarrow \ell_{12,3}^{*} \alpha,
$$

and thus precisely the datum (ii) we need for the $\Gamma$-equivariant structure. It is then easy to observe that the pullback of the commutative diagram (A.4.3) along $f_{4}$ is, using rules (A.4.13), precisely the diagram (5.1) in Definition 5.1. Thus, $(\mathcal{G}, \alpha, \beta)$ is a $\Gamma$-equivariant gerbe relative to the zero 2 -form. In the same way one verifies, using (A.4.11)-(A.4.13), that 1-isomorphisms and 2-isomorphisms in $\operatorname{Des}(\omega)$ pull back to 1-isomorphisms and 2-isomorphisms between $\Gamma$-equivariant gerbes, respectively. This defines a functor

$$
f^{*}: \operatorname{Des}(\omega) \rightarrow \operatorname{Grb}^{\nabla}(M)_{0}^{\Gamma} .
$$

This functor is an equivalence, because the maps $f_{p}$ are diffeomorphisms. Indeed, if $(\mathcal{G}, \alpha, \beta)$ is a $\Gamma$-equivariant gerbe then, using (A.4.11)-(A.4.13) again, one observes that $\mathcal{C}:=\left(f_{2}^{-1}\right)^{*} \alpha$ and $\lambda:=\left(f_{3}^{-1}\right)^{*} \beta$ make up a descent object $(\mathcal{G}, \mathcal{C}, \lambda)$, and analogously for 1-isomorphisms and 2-isomorphisms.

5. Proof of Lemma 5.4. For $\tilde{\rho}_{\mathcal{A}}$ and $\tilde{\ell}$ defined by Eqs. (5.8) and (5.9), one obtains by virtue of relations (5.7) and (3.27):

$$
\begin{aligned}
& \left(\tilde{\rho}_{\mathcal{A}}\right)_{\tilde{1} \tilde{2}}(\gamma,(p, m))=\left(\tilde{\ell}^{*} \tilde{\rho}_{\mathcal{A}}\right)(\gamma,(p, m))=\left(\ell_{1,3}^{*} \tilde{\rho}_{A d_{\gamma}(\mathcal{A}-\Theta(\gamma))}\right)(\gamma, p, m) \\
& =\left(\exp \left[-\iota_{\bar{\Theta}(\gamma)}\right]\left(\ell_{\gamma}\right)_{3}^{*} \tilde{\rho}_{A d_{\gamma}(\mathcal{A}-\Theta(\gamma))}\right)(p, m) \\
& =\left(\left(\ell_{\gamma}\right)_{3}^{*} \tilde{\rho}_{A d_{\gamma}(\mathcal{A}-\Theta(\gamma))}\right)(p, m)-\left(\iota_{\bar{\Theta}(\gamma)}\left(\ell_{\gamma}\right)_{3}^{*} \tilde{\rho}_{A d_{\gamma}(\mathcal{A}-\Theta(\gamma))}\right)(p, m) \text {. }
\end{aligned}
$$

The $2^{\text {nd }}$ of relations (3.24) implies further that

$$
\begin{aligned}
& \left(\left(\ell_{\gamma}\right)_{3}^{*} \tilde{\rho}_{\left.A d_{\gamma}(\mathcal{A}-\Theta(\gamma))\right)(p, m)}\right. \\
& \quad=\left\{\left(\ell_{\gamma}\right)_{3}^{*}\left[-v\left(A d_{\gamma}(\mathcal{A}-\Theta(\gamma))\right)+\frac{1}{2} \iota \frac{}{A d_{\gamma}(\mathcal{A}-\Theta(\gamma))} v\left(A d_{\gamma}(\mathcal{A}-\Theta(\gamma))\right)\right]\right\}(p, m) \\
& \quad=\left[-v(\mathcal{A}-\Theta(\gamma))+\frac{1}{2} \iota \frac{\mathcal{A}-\Theta(\gamma)}{} v(\mathcal{A}-\Theta(\gamma))\right](p, m) .
\end{aligned}
$$

Hence,

$$
\begin{aligned}
& {\left[\left(\ell_{\gamma}\right)_{3}^{*} \tilde{\rho}_{A d_{\gamma}(\mathcal{A}-\Theta(\gamma))}\right](p, m)} \\
& \quad=\left[-v(\mathcal{A})+\frac{1}{2} \iota_{\overline{\mathcal{A}}} v(\mathcal{A})+v(\Theta(\gamma))+\frac{1}{2} \iota_{\bar{\Theta}(\gamma)} v(\Theta(\gamma))\right.
\end{aligned}
$$




$$
\begin{aligned}
& \left.-\frac{1}{2} \iota_{\bar{\Theta}(\gamma)} v(\mathcal{A})-\frac{1}{2} \iota_{\overline{\mathcal{A}}} v(\Theta(\gamma))\right](p, m) \\
= & {\left[-v(\mathcal{A})+\frac{1}{2} \iota_{\overline{\mathcal{A}}} v(\mathcal{A})+v(\Theta(\gamma))+\frac{1}{2} \iota_{\bar{\Theta}(\gamma)} v(\Theta(\gamma))-\iota_{\bar{\Theta}(\gamma)} v(\mathcal{A})\right](p, m), }
\end{aligned}
$$

where the last equality follows from the right one of relations (3.6). Consequently,

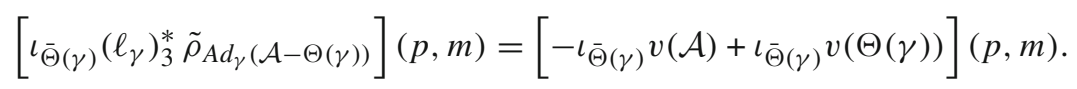

Subtracting the last expression from the previous one, we infer from Eq. (A.5.1) the relation

$$
\begin{aligned}
\left(\tilde{\rho}_{\mathcal{A}}\right)_{\tilde{1} \tilde{2}}(\gamma,(p, m)) & =\left[-v(\mathcal{A})+\frac{1}{2} \iota_{\overline{\mathcal{A}}} v(\mathcal{A})+v(\Theta(\gamma))-\frac{1}{2} \iota_{\bar{\Theta}(\gamma)} v(\Theta(\gamma))\right](p, m) \\
& =\tilde{\rho}_{\mathcal{A}}(p, m)-\rho(\gamma, m) .
\end{aligned}
$$

This is the identity claimed by Lemma 5.4

6. Construction of flat gerbes from characters. Let $\Gamma$ be a connected Lie group and $\omega: M \rightarrow M^{\prime}$ a left principal $\Gamma$-bundle. We shall assume that $M$ is also connected. One has $\Gamma=\tilde{\Gamma} / \tilde{Z}_{\Gamma}$ where $\tilde{\Gamma}$ is the covering group of $\Gamma$ and $\tilde{Z}_{\Gamma}$ is a subgroup of the center of $\tilde{\Gamma}$ and is naturally identified with the fundamental group of $\Gamma$. Note that $H^{1}(\Gamma, U(1)) \cong \tilde{Z}_{\Gamma}^{*}$. To each character $\chi \in \tilde{Z}_{\Gamma}^{*}$, there corresponds a flat line bundle $L_{\chi}$ composed of classes $[\tilde{\gamma}, u]_{\chi}$ of the equivalence relation on $\tilde{\Gamma} \times \mathbf{C}$

$$
(\tilde{\gamma}, u) \underset{\chi}{\sim}\left(\tilde{\gamma} z^{-1}, \chi(z) u\right)
$$

for $z \in \tilde{Z}_{\Gamma}$. We can associate to this line bundle $L_{\chi}$ a flat gerbe $\mathcal{G}_{\chi}=(Y, B, L, \mu)$ over $M^{\prime}$ using the geometric description of gerbes mentioned in the beginning of Sect. 7. We shall take $Y=M$ with the canonical projection on $M^{\prime}$ and a vanishing curving $B=0$. The fiber products $Y^{[p]}=M^{[p]}$ may be naturally identified with $\Gamma^{p-1} \times M$ by the map $f_{p}$ given by Eq. (A.4.10). For the line bundle $L$ we shall take the pullback of $L_{\chi}$ along the map $Y^{[2]} \ni(\gamma m, m) \mapsto \gamma \in \Gamma$. The groupoid multiplication $\mu$ is then induced by the map

$$
\left[\tilde{\gamma}_{1}, u_{1}\right]_{\chi} \otimes\left[\tilde{\gamma}_{2}, u_{2}\right]_{\chi} \longmapsto\left[\tilde{\gamma}_{1} \tilde{\gamma}_{2}, u_{1} u_{2}\right]_{\chi} .
$$

It is easy to show that the pullback gerbe $\omega^{*} \mathcal{G}_{\chi}$ is 1 -isomorphic to the trivial gerbe $\mathcal{I}_{0}$ on $M$ and that $\mathcal{G}_{\chi}$ is 1-isomorphic to the trivial gerbe on $M^{\prime}$ if and only the flat line bundle $L_{\chi}$ extends from a fiber of the bundle $\omega: M \rightarrow M^{\prime}$ to $M$.

The 1-isomorphism class of the flat gerbe $\mathcal{G}_{\chi}$ gives the element of $H^{2}\left(M^{\prime}, U(1)\right)$ associated by the middle homomorphism $\tau$ in the exact sequence (6.59) to the element of $H^{1}(\Gamma, U(1))$ identified with the character $\chi$ of $\tilde{Z}_{\Gamma}$.

7. Behavior of isomorphism $\alpha$ under groupoid multiplication. We verify here that, for the line bundle isomorphism $\alpha$ constructed in Sect. 7.2, the two composed isomorphisms (7.49) and (7.50) coincide so that $\alpha$ defines a 1-isomorphism between the gerbes $\left(\mathcal{G}_{k}\right)_{12}$ and $\mathcal{I}_{\rho} \otimes\left(\mathcal{G}_{k}\right)_{2}$ over the product group $\Gamma \times \tilde{G}$. Similarly as for $W_{12}^{[2]}$, see Eq. (7.39), we have: 


$$
W_{12}^{[3]}=\left(\tilde{G} \times Y^{[3]}\right) / \tilde{Z} .
$$

Over $\left(\tilde{G} \times Y_{i_{1} j_{1} i_{2} j_{2} i_{3} j_{3}}\right) / \tilde{Z} \subset W_{12}^{[3]}$, consider elements $\ell_{i_{1} i_{2}} \ldots \ell_{j_{1} j_{3}}$ in the respective fibers of $L$. The composition (7.49) of line bundle isomorphisms is induced by the map

$$
\begin{aligned}
& \left(\tilde{\gamma}, \ell_{i_{1} i_{2}} \otimes \ell_{i_{2} i_{3}} \otimes \ell_{i_{3} j_{3}}\right) \stackrel{I d \otimes \tilde{\alpha}_{2,3}}{\longmapsto}\left(\tilde{\gamma}, \ell_{i_{1} i_{2}} \otimes \ell_{i_{2} j_{2}} \otimes \ell_{j_{2} j_{3}}\right) \\
& \quad \stackrel{\tilde{\alpha}_{1,2} \otimes I d}{\longmapsto}\left(\tilde{\gamma}, \ell_{i_{1} j_{1}} \otimes \ell_{j_{1} j_{2}} \otimes \ell_{j_{2} j_{3}}\right) \stackrel{I d \times\left(I d \otimes\left(\mu_{2}\right)_{1,2,3}\right)}{\longmapsto}\left(\tilde{\gamma}, \ell_{i_{1} j_{1}} \otimes \ell_{j_{1} j_{3}}\right)
\end{aligned}
$$

with $\mu\left(\ell_{i_{2} i_{3}} \otimes \ell_{i_{3} j_{3}}\right)=\mu\left(\ell_{i_{2} j_{2}} \otimes \ell_{j_{2} j_{3}}\right), \mu\left(\ell_{i_{1} i_{2}} \otimes \ell_{i_{2} j_{2}}\right)=\mu\left(\ell_{i_{1} j_{1}} \otimes \ell_{j_{1} j_{2}}\right)$ and $\mu\left(\ell_{j_{1} j_{2}} \otimes\right.$ $\left.\ell_{j_{2} j_{3}}\right)=\ell_{j_{1} j_{3}}$. The associativity of the groupoid multiplication $\mu$ then implies that

$$
\begin{gathered}
\mu\left(\ell_{i_{1} i_{2}} \otimes \mu\left(\ell_{i_{2} i_{3}} \otimes \ell_{i_{3} j_{3}}\right)\right)=\mu\left(\ell_{i_{1} i_{2}} \otimes \mu\left(\ell_{i_{2} j_{2}} \otimes \ell_{j_{2} j_{3}}\right)\right)=\mu\left(\mu\left(\ell_{i_{1} i_{2}} \otimes \ell_{i_{2} j_{2}}\right) \otimes \ell_{j_{2} j_{3}}\right) \\
=\mu\left(\mu\left(\ell_{i_{1} j_{1}} \otimes \ell_{j_{1} j_{2}}\right) \otimes \ell_{j_{2} j_{3}}\right)=\mu\left(\ell_{i_{1} j_{1}} \otimes \mu\left(\ell_{j_{1} j_{2}} \otimes \ell_{j_{2} j_{3}}\right)\right)=\mu\left(\ell_{i_{1} j_{1}} \otimes \ell_{j_{1} j_{3}}\right) .
\end{gathered}
$$

Similarly, the composition (7.50) descends from the map

$$
\left(\tilde{\gamma}, \ell_{i_{1} i_{2}} \otimes \ell_{i_{2} i_{3}} \otimes \ell_{i_{3} j_{3}}\right) \stackrel{I d \times\left(\left(\mu_{12}\right)_{1,2,3} \otimes I d\right)}{\longmapsto}\left(\tilde{\gamma}, \ell_{i_{1} i_{3}} \otimes \ell_{i_{3} j_{3}}\right) \stackrel{\tilde{\alpha}_{1,3}}{\longmapsto}\left(\tilde{\gamma}, \ell_{i_{1} j_{1}} \otimes \ell_{j_{1} j_{3}}\right)
$$

with $\mu\left(\ell_{i_{1} i_{2}} \otimes \ell_{i_{2} i_{3}}\right)=\ell_{i_{1} i_{3}}$ and $\mu\left(\ell_{i_{1} i_{3}} \otimes \ell_{i_{3} j_{3}}\right)=\mu\left(\ell_{i_{1} j_{1}} \otimes \ell_{j_{1} j_{3}}\right)$. Now

$$
\mu\left(\mu\left(\ell_{i_{1} i_{2}} \otimes \ell_{i_{2} i_{3}}\right) \otimes \ell_{i_{3} j_{3}}\right)=\mu\left(\ell_{i_{1} i_{3}} \otimes \ell_{i_{3} j_{3}}\right)=\mu\left(\ell_{i_{1} j_{1}} \otimes \ell_{j_{1} j_{3}}\right) .
$$

Comparison between the relations (A.7.3) and (A.7.5) and the use of the associativity of $\mu$ show that the target elements of (A.7.2) and (A.7.4) coincide if the initial elements are the same. That demonstrates the identity of two composed line bundle isomorphisms (7.49) and (7.50).

8. Commutativity of diagram (7.74). We shall prove that diagram (7.74) of isomorphisms of line bundles over $W_{123}^{[2]}$ is commutative. Over subspace $\left(\tilde{G}^{2} \times Y_{i_{1} j_{1} k_{1} i_{2} j_{2} k_{2}}\right) / \tilde{Z}^{2} \subset$ $W_{123}^{[2]}$, with notations similar to those in the previous Appendix, the top line of the diagram is induced by the composite map

$$
\begin{aligned}
\left(\tilde{\gamma}_{1}, \tilde{\gamma}_{2}, \ell_{i_{1} i_{2}} \otimes \ell_{i_{2} j_{2}} \otimes \ell_{j_{2} k_{2}}\right) & \stackrel{\tilde{\alpha}_{1,23} \otimes I d}{\longmapsto}\left(\tilde{\gamma}_{1}, \tilde{\gamma}_{2}, \ell_{i_{1} j_{1}} \otimes \ell_{j_{1} j_{2}} \otimes \ell_{j_{2} k_{2}}\right) \\
& \longmapsto d \otimes \tilde{\alpha}_{2,3} \\
& \left.\longmapsto \tilde{\gamma}_{1}, \tilde{\gamma}_{2}, \ell_{i_{1} j_{1}} \otimes \ell_{j_{1} k_{1}} \otimes \ell_{k_{1} k_{2}}\right)
\end{aligned}
$$

with $\mu\left(\ell_{i_{1} i_{2}} \otimes \ell_{i_{2} j_{2}}\right)=\mu\left(\ell_{i_{1} j_{1}} \otimes \ell_{j_{1} j_{2}}\right)$ and $\mu\left(\ell_{j_{1} j_{2}} \otimes \ell_{j_{2} k_{2}}\right)=\mu\left(\ell_{j_{1} k_{1}} \otimes \mu_{k_{1} k_{2}}\right)$ which imply that

$$
\begin{aligned}
\mu\left(\mu\left(\ell_{i_{1} i_{2}} \otimes \ell_{i_{2} j_{2}}\right) \otimes \ell_{j_{2} k_{2}}\right) & =\mu\left(\mu\left(\ell_{i_{1} j_{1}} \otimes \ell_{j_{1} j_{2}}\right) \otimes \ell_{j_{2} k_{2}}\right) \\
& =\mu\left(\ell_{i_{1} j_{1}} \otimes \mu\left(\ell_{j_{1} j_{2}} \otimes \ell_{j_{2} k_{2}}\right)\right) \\
& =\mu\left(\ell_{i_{1} j_{1}} \otimes \mu\left(\ell_{j_{1} k_{1}} \otimes \ell_{k_{1} k_{2}}\right)\right) .
\end{aligned}
$$


The bottom line of the diagram (7.74) descends from the map

$$
\left(\tilde{\gamma}_{1}, \tilde{\gamma}_{2}, \ell_{i_{1} i_{2}} \otimes \ell_{i_{2} k_{2}}\right) \stackrel{\tilde{\alpha}_{12,3}}{\longrightarrow}\left(\tilde{\gamma}_{1}, \tilde{\gamma}_{2}, \ell_{i_{1} k_{1}} \otimes \ell_{k_{1} k_{2}}\right)
$$

with

$$
\mu\left(\ell_{i_{1} i_{2}} \otimes \ell_{i_{2} k_{2}}\right)=\mu\left(\ell_{i_{1} k_{1}} \otimes \ell_{k_{1} k_{2}}\right)
$$

Assuming that

$$
\begin{aligned}
& \left(\tilde{\gamma}_{1}, \tilde{\gamma}_{2}, \ell_{i_{2} k_{2}}\right)=\tilde{\beta}\left(\tilde{\gamma}_{1}, \tilde{\gamma}_{2}, \ell_{i_{2} j_{2}} \otimes \ell_{j_{2} k_{2}}\right) \text { and } \\
& \left(\tilde{\gamma}_{1}, \tilde{\gamma}_{2}, \ell_{i_{1} k_{1}}\right)=\tilde{\beta}\left(\tilde{\gamma}_{1}, \tilde{\gamma}_{2}, \ell_{i_{1} j_{1}} \otimes \ell_{j_{1} k_{1}}\right),
\end{aligned}
$$

i.e. that $\ell_{i_{2} k_{2}}=\mu\left(\ell_{i_{2} j_{2}} \otimes \ell_{j_{2} k_{2}}\right)$ and $\ell_{i_{1} k_{1}}=\mu\left(\ell_{i_{1} j_{1}} \otimes \ell_{j_{1} k_{1}}\right)$, we infer from comparison between Eqs. (A.8.4) and (A.8.2) that the target elements of (A.8.1) and (A.8.3) coincide, establishing the commutativity of diagram (7.74).

9. Proof of the equality of isomorphisms (7.76) and (7.77). Similarly as before, one may identify

$$
W_{1234}=\left(\tilde{G}^{3} \times Y^{[4]}\right) / \tilde{Z}^{3}
$$

with the action of $\tilde{Z}^{3}$ given by

$$
\begin{aligned}
& \left(\tilde{\gamma}_{1}, \tilde{\gamma}_{2}, \tilde{\gamma}_{3},\left(y, y^{\prime}, y^{\prime \prime}, y^{\prime \prime \prime}\right)\right) \\
& \quad \longmapsto\left(z_{1} \tilde{\gamma}_{1}, z_{2} \tilde{\gamma}_{2}, z_{3} \tilde{\gamma}_{3},\left(y\left(z_{1} z_{2} z_{3}\right)^{-1}, y^{\prime}\left(z_{2} z_{3}\right)^{-1}, y^{\prime \prime} z_{3}^{-1}, y^{\prime \prime \prime}\right)\right) .
\end{aligned}
$$

The different pullbacks of the bundle $E$ over $W_{12}$ to $W_{1234}$ may be identified as

$$
\begin{aligned}
& E_{1,234} \cong\left(\tilde{G}^{3} \times L_{1,2}\right) / \tilde{Z}^{3}, \quad E_{2,34} \cong\left(\tilde{G}^{3} \times L_{2,3}\right) / \tilde{Z}^{3}, \quad E_{3,4} \cong\left(\tilde{G}^{3} \times L_{3,4}\right) / \tilde{Z}^{3}, \\
& E_{23,4} \cong\left(\tilde{G}^{3} \times L_{2,4}\right) / \tilde{Z}^{3}, \quad E_{12,34} \cong\left(\tilde{G}^{3} \times L_{1,3}\right) / \tilde{Z}^{3}, \quad E_{123,4} \cong\left(\tilde{G}^{3} \times L_{1,4}\right) / \tilde{Z}^{3},
\end{aligned}
$$

with appropriate actions of $\tilde{Z}^{3}$ and appropriate modifications of the connection of the pullbacks of $L$. If $\left(y, y^{\prime}, y^{\prime \prime}, y^{\prime \prime \prime}\right) \in Y_{i j k l} \subset Y^{[4]}$ and $\ell_{i j} \in L_{\left(y, y^{\prime}\right)} \subset L_{1,2}, \ldots \ldots, \ell_{i l} \in$ $L_{\left(y, y^{\prime \prime \prime}\right)} \subset L_{1,4}$, then the composition (7.76) of the line bundle isomorphisms is induced by the map

$$
\begin{gathered}
\left(\tilde{\gamma}_{1}, \tilde{\gamma}_{2}, \tilde{\gamma}_{3}, \ell_{i j} \otimes \ell_{j k} \otimes \ell_{k l}\right) \stackrel{I d \times I d \otimes \tilde{\beta}_{2,3,4}}{\longmapsto}\left(\tilde{\gamma}_{1}, \tilde{\gamma}_{2}, \tilde{\gamma}_{3}, \ell_{i j} \otimes \ell_{j l}\right) \\
\stackrel{I d \times \tilde{\beta}_{1,23,4}}{\longmapsto}\left(\tilde{\gamma}_{1}, \tilde{\gamma}_{2}, \tilde{\gamma}_{3}, \ell_{i l}\right)
\end{gathered}
$$

with $\ell_{j l}=\mu\left(\ell_{j k} \otimes \ell_{k l}\right)$ and $\ell_{i l}=\mu\left(\ell_{i j} \otimes \ell_{j l}\right)=\mu\left(\ell_{i j} \otimes \mu\left(\ell_{j k} \otimes \ell_{k l}\right)\right)$. On the other hand, the composition (7.77) is given by

$$
\begin{gathered}
\left(\tilde{\gamma}_{1}, \tilde{\gamma}_{2}, \tilde{\gamma}_{3}, \ell_{i j} \otimes \ell_{j k} \otimes \ell_{k l}\right) \stackrel{I d \times \tilde{\beta}_{1,2,34} \otimes I d}{\longmapsto}\left(\tilde{\gamma}_{1}, \tilde{\gamma}_{2}, \tilde{\gamma}_{3}, \ell_{i k} \otimes \ell_{k l}\right) \\
\stackrel{I d \times \tilde{\beta}_{12,3,4}}{\longmapsto}\left(\tilde{\gamma}_{1}, \tilde{\gamma}_{2}, \tilde{\gamma}_{3}, \ell_{i l}\right)
\end{gathered}
$$

with $\ell_{i k}=\mu\left(\ell_{i j} \otimes \ell_{j k}\right)$ and $\ell_{i l}=\mu\left(\ell_{i k} \otimes \ell_{k l}\right)=\mu\left(\mu\left(\ell_{i j} \otimes \ell_{j k}\right) \otimes \ell_{k l}\right)$. Using the associativity of $\mu$, we infer that the two compositions give the same line-bundle isomorphism. 


\section{References}

1. Alvarez, O.: Topological quantization and cohomology. Commun. Math. Phys. 100, 279-309 (1985)

2. Bardakci, K., Rabinovici, E., Säring, B.: String models with $c<1$ components. Nucl. Phys. B 299, 151-182 (1988)

3. Bertlmann, R.A.: Anomalies in Quantum Field Theory. Oxford-New York: Oxford University Press, 2000

4. Brown, K.S.: Cohomology of Groups. Berlin-Heidelberg-New-York: Springer, 1982

5. Carey, A.L., Johnson, S., Murray, M.K., Stevenson, D., Wang, B.L.: Bundle gerbes for Chern-Simons and Wess-Zumino-Witten theories. Commun. Math. Phys. 259, 577-613 (2005)

6. Carey, A.L., Murray, M.K., Wang, B.L.: Higher bundle gerbes and cohomology classes in gauge theories. J. Geom. Phys. 21, 183-197 (1997)

7. Chatterjee, D.S.: On gerbes. Ph.D. thesis, Trinity College, Cambridge, 1998

8. Di Vecchia, P., Durhuus, B., Petersen, J.L.: The Wess-Zumino action in two dimensions and non-abelian bosonization. Phys. Lett. B 144, 245-249 (1984)

9. Dunbar, D.C., Joshi, K.G.: Maverick examples of coset conformal field theories. Mod. Phys. Lett. A 8, 2803-2814 (1993)

10. Dubrovin, B.A., Fomenko, A.T., Novikov, S.P.: Modern Geometry - Methods and Applications. Part III, Introduction to Homology Theory. Berlin-Heidelberg-New-York: Springer, 1990

11. Fabbrichesi, M.: Cancellation of global anomalies in spontaneously broken gauge theories. Pramana 62, 725-727 (2004)

12. Figueroa-O'Farrill, J.M., Mohammedi, N.: Gauging the Wess-Zumino term of a sigma model with boundary. JHEP 08, 086 (2005)

13. Figueroa-O'Farrill, J.M., Stanciu, S.: Equivariant cohomology and gauged bosonic $\sigma$-models, http:// arXiv.org/abs/hep-th/9407149v3, 1994

14. Figueroa-O'Farrill, J.M., Stanciu, S.: Gauged Wess-Zumino terms and equivariant cohomology. Phys. Lett. B 341, 153-159 (1994)

15. Felder, G., Gawędzki, K., Kupiainen, A.: Spectra of Wess-Zumino-Witten models with arbitrary simple groups. Commun. Math. Phys. 117, 127-158 (1988)

16. Fuchs, J., Schellekens, B., Schweigert, C.: The resolution of field identification fixed points in diagonal coset theories. Nucl. Phys. B 461, 371-406 (1996)

17. Gawędzki, K.: Topological actions in two-dimensional quantum field theories. In: Hooft, G.'t, Jaffe, A., Mack, G., Mitter, P.K., Stora, R. (eds.) Non-perturbative Quantum Field Theory. New York: Plenum Press, 1988, pp. 101-142

18. Gawȩdzki, K.: Conformal field theory. In: Séminaire Bourbaki, Exposé 704, Astérisque 177/178, 95-126 (1989)

19. Gawędzki, K.: Geometry of Wess-Zumino-Witten models of conformal field theory. In: Recent Advances in Field Theory. Binétruy, P., Girardi, G., Sorba, P. (eds.) Nucl. Phys. (Proc. Suppl.) B 18, 78-91 (1990)

20. Gawȩdzki, K.: Abelian and non-Abelian branes in WZW models and gerbes. Commun. Math. Phys. 258, 23-73 (2005)

21. Gawȩdzki, K., Kupiainen, A.: $G / H$ conformal field theory from gauged WZW model. Phys. Lett. B 215, 119-123 (1988)

22. Gawȩdzki, K., Kupiainen, A.: Coset construction from functional integral. Nucl. Phys. B 320, 625-668 (1989)

23. Gawȩdzki, K., Reis, N.: WZW branes and gerbes. Rev. Math. Phys. 14, 1281-1334 (2002)

24. Gawȩdzki, K., Reis, N.: Basic gerbe over non simply connected compact groups. J. Geom. Phys. 50, 28-55 (2004)

25. Gawȩdzki, K., Waldorf, K.: Polyakov-Wiegmann formula and multiplicative gerbes. JHEP 09, 073 (2009)

26. Gawędzki, K., Suszek, R.R., Waldorf, K.: WZW orientifolds and finite group cohomology. Commun. Math. Phys. 284, 1-49 (2008)

27. Gawȩdzki, K., Suszek, R.R., Waldorf, K.: Bundle gerbes for orientifold sigma models, http://arXiv.org/ abs/0809.5125v2 [math-ph], 2008

28. Gepner, D., Witten, E.: String theory on group manifolds. Nucl. Phys. B 278, 493-549 (1986)

29. Goddard, P.: Infinite dimensional Lie algebras: representations and applications. In: WSGP5, Proceedings of the Winter School "Geometry and Physics" Frolík, Z., Souček, V., Vinárek, J. (eds.), Palermo: Circolo Matematico di Palermo, 1985, pp. 73-107

30. Goddard, P., Kent, A., Olive, D.: Virasoro Algebras and Coset Space Models. Phys. Lett. B 152, 88-92 (1985)

31. Gomi, K.: Equivariant smooth Deligne cohomology. Osaka J. Math. 42, 309-337 (2005)

32. Hitchin, N.J.: Lectures on special Lagrangian submanifolds. In: Winter School on Mirror Symmetry, Vector Bundles and Lagrangian Submanifolds. Vafa, C., Yau, S.-T. (eds.) AMS/IP Stud. Adv. Math. Vol. 23, Providence, RI: Amer. Math. Soc., 2001, pp. 151-182

33. Hori, K.: Global aspects of gauged Wess-Zumino-Witten models. Commun. Math. Phys. 182, 1-32 (1996) 
34. Hull, C.M.: Global aspects of T-duality, gauged sigma models and T-folds. JHEP 10, 057 (2007)

35. Hull, C.M.: Doubled geometry and T-folds. JHEP 07, 080 (2007)

36. Hull, C.M., Spence, B.: The gauged nonlinear sigma model with Wess-Zumino term. Phys. Lett. B 232, 204-210 (1989)

37. Jack, I., Jones, D.R.T., Mohammedi, N., Osborn, H.: Gauging the general $\sigma$-model with a Wess-Zumino term. Nucl. Phys. B 332, 359-379 (1990)

38. Kalkman, J.: BRST model for equivariant cohomology and representatives for the equivariant Thom class. Commun. Math. Phys. 153, 447-463 (1993)

39. Kac, V.G.: Infinite dimensional Lie algebras, $2^{\text {nd }}$ edition, Cambridge: Cambridge University Press, 1985

40. Karabali, D., Park, Q., Schnitzer, H.J., Yang, Z.: A GKO construction based on a path integral formulation of gauged Wess-Zumino-Witten actions. Phys. Lett. B 216, 307-312 (1989)

41. Kreuzer, M., Schellekens, A.N.: Simple currents versus orbifolds with discrete torsion - a complete classification. Nucl. Phys. B 411, 97-121 (1994)

42. Meinrenken, E.: The basic gerbe over a compact simple Lie group. Enseign. Math. 49, 307-333 (2003)

43. Murray, M.K.: Bundle gerbes. J. London Math. Soc. 54(2), 403-416 (1996)

44. Murray, M.K., Stevenson, D.: Bundle gerbes: stable isomorphisms and local theory. J. London Math. Soc. 62(2), 925-937 (2000)

45. Nikolaus, T.: Äquivariante Gerben und Abstieg. Diploma thesis, University of Hamburg, 2009

46. Petersen, J.L.: Non-abelian chiral anomalies and Wess-Zumino effective actions. Acta Phys. Polon. B 16, 271-300 (1985)

47. Schellekens, A.N., Yankielowicz, S.: Field identification fixed points in the coset construction. Nucl. Phys. B 334, 67-102 (1990)

48. Schreiber, U., Schweigert, C., Waldorf, K.: Unoriented WZW models and holonomy of bundle gerbes. Commun. Math. Phys. 274, 31-64 (2007)

49. Serre, J.-P.: Homologie singulière des espaces fibrés. Ann. of Math. 54, 425-505 (1951)

50. Stevenson, D.: The geometry of bundle gerbes. Ph.D. thesis, University of Adelaide, 2000, http://arXiv. org/abs/0004117v1 [math.DG], 2000

51. Hooft, G.'t.: Naturalness, chiral symmetry, and spontaneous chiral symmetry breaking. In: Recent Developments in Gauge Theories. Hooft, G.'t, Itzykson, C., Jaffe, A., Lehmann, H., Mitter, P.K., Singer, I.M., Stora, R. (eds.), New York: Plenum Press, 1980

52. Tu, J.-L.: Groupoid cohomology and extensions. Trans. Amer. Math. Soc. 358, 4721-4747 (2006)

53. Vafa, C.: Modular invariance and discrete torsion on orbifolds. Nucl. Phys. B 273, 592-606 (1986)

54. Waldorf, K.: More morphisms between bundle gerbes. Theory Appl. Categ. 18, 240-273 (2007)

55. Waldorf, K.: Multiplicative bundle gerbes with connection. Diff. Geom. Appl 28(3), 313-340 (2010)

56. Weinberg, S.: The Quantum Theory of Fields, Vol. 2: Modern Applications. Cambridge: Cambridge University Press, 1996

57. Wess, J., Zumino, B.: Consequences of anomalous Ward identies. Phys. Lett. B 37, 95-97 (1971)

58. Witten, E.: An SU(2) anomaly. Phys. Lett. B 117, 324-328 (1982)

59. Witten, E.: Non-abelian bosonization in two dimensions. Commun. Math. Phys. 92, 455-472 (1984)

60. Witten, E.: On holomorphic factorization of WZW and coset models. Commun. Math. Phys. 144, 189-212 (1992)

61. Wu, S.: Cohomological obstructions to the equivariant extension of closed invariant forms. J. Geom. Phys. 10, 381-392 (1993)

Communicated by A. Kapustin 